\title{
A Relationship Focused Approach to Epistemic Injustice in Global Development Theory and Practice
}

\author{
by
}

\author{
Holly Longair
}

A thesis submitted to the Faculty of Graduate and Postdoctoral Affairs in partial fulfillment of the requirements for the degree of

\author{
Master of Arts
}

in

Philosophy

Carleton University

Ottawa, Ontario

(C) 2017, Holly Longair 


\begin{abstract}
$\underline{\text { Abstract }}$
Miranda Fricker's theory of epistemic injustice articulates the connections between ethics and epistemology that come to light in the harms caused when someone is wronged in their capacities as a knower. Although Fricker's account has provided an important contribution to the academic literature, its many flaws mean that a new approach to epistemic injustice is needed. This new account will focus more on structures of society, context, and relationships to better understand how epistemic injustices happen. This new approach will then be applied to the broad context of global development, and to the specific case study of Cuba's education and health care policies. The goal of this project is to show that epistemic injustice interferes with effective and ethical global development work aimed at improving the well-being of people, and how taking a more structural, contextual, and relational approach will mitigate the harms of epistemic injustice.
\end{abstract}




\section{$\underline{\text { Acknowledgements }}$}

First and foremost, I want to thank my supervisor, Christine Koggel, for your enthusiasm for this project and for your never-ending support in every aspect of my academic and professional development. I couldn't have done it without you.

Thank you also to Jay Drydyk for your encouragement and mentoring throughout the last two years, to my committee (David Mattheson and Audra Diptee) for your constructive evaluation of my work, and to Sandy Kirkpatrick for being the source of all administrative knowledge and saving me many times.

Thank you to Arielle and Amy for the many hours sharing our ideas and our struggles, and to my entire cohort (Arielle, Amy, Jo, Sebastien, Dan, and Courtney) for your input in the early stages of this project and for your continuing support and solidarity over the last two years. Thank you to my roommates, Breanna and Nora (and Derrick too), for always being willing to listen and for being understanding about how crazy this year was. To Ben, thank you for being here for me. To my fellow adventurers (Asha, Nevin, Jake, and Torey), thank you for providing a haven where I could escape for a few hours every week and fight demons rather than fighting to get words on paper.

My family has also played an important part in helping me get me to where I am. Thank you to my mom, Trish, for being an inspiration and an example of taking control of your life to follow your dreams; to my dad, Rob, for instilling in me a love of learning and for always pushing me to do my best while reminding me to also enjoy life; and to my brother, James, for listening to me talk about my studies and research even if you had no interest. 
Last but far from least, thank you to those whose teaching has led me to this point. To Mark Smith, thank you for being the first to introduce me to many of the ideas in this paper about epistemic injustice, and to Susan Babbitt, thank you for introducing me to non-European philosophy and a different way of looking at Cuba. Without the support from you both during my undergraduate degree I would not be where I am today. Finally, to Glyn Hughes, thank you for always pushing me to think critically and for fueling my passion for social justice. This may not be the path you expected me to pursue, but it is your lessons that led me here. You are missed. 


\section{Table of Contents}

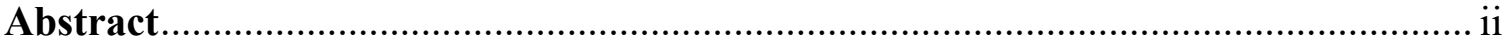

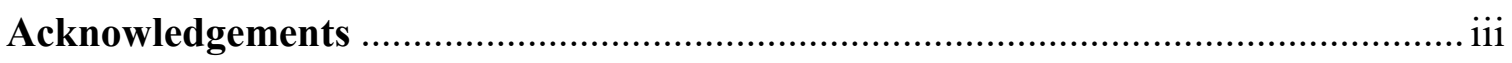

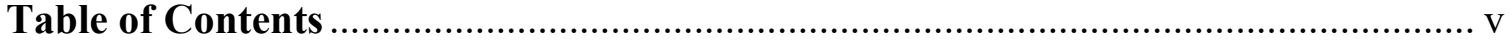

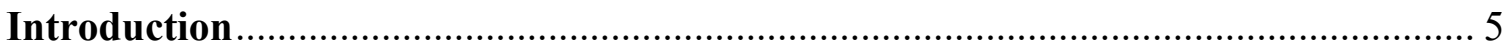

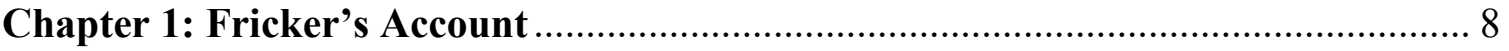

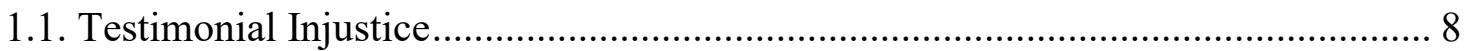

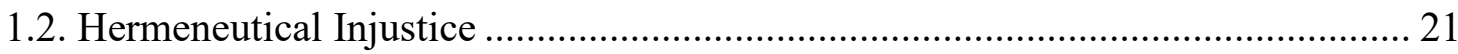

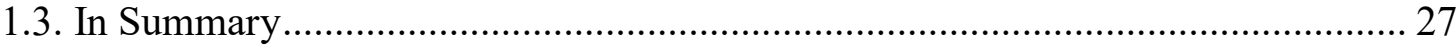

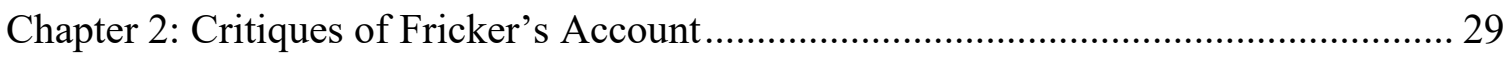

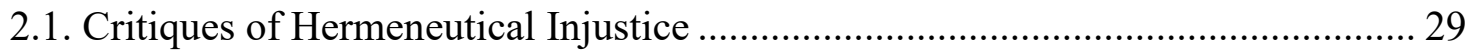

2.1.1. The Problem with the "Collective Hermeneutical Resource"........................ 30

2.1.2. False Consciousness and the Problem with Imposing Concepts.................... 36

2.2. What Fricker is Missing .......................................................................... 40

2.2.1. Epistemic Activities that are not Testimony ................................................. 41

2.2.2. Problems with the Definition of Testimonial Injustice ................................ 43

2.2.3. Discrediting Privileged Advocates ............................................................ 46

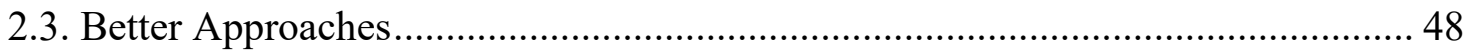

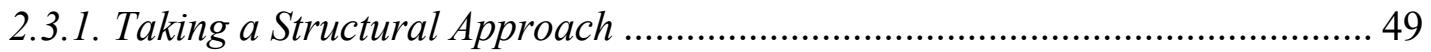

2.3.2. Paying More Attention to Relationships ...................................................... 51

2.4. The Problem with Categories.................................................................... 53

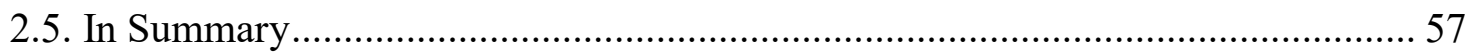

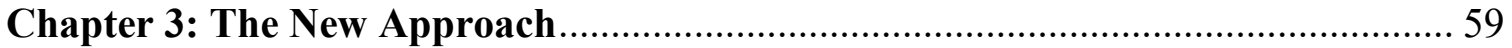

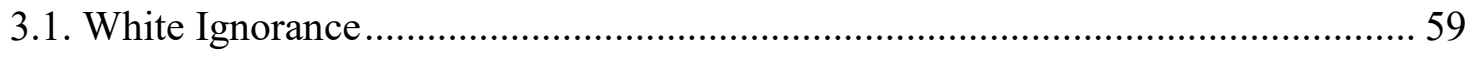

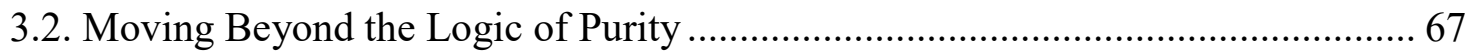


3.3. Moving Towards a Complex View of the Self.

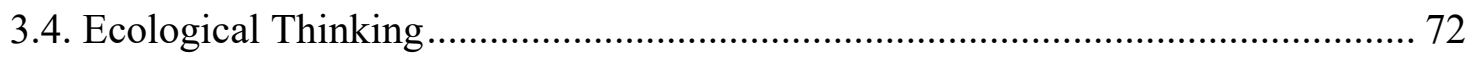

3.5. A New Approach to Epistemic Injustice ................................................... 74

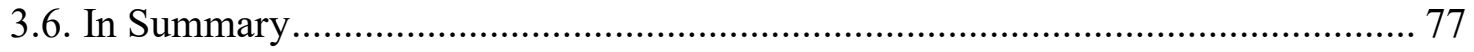

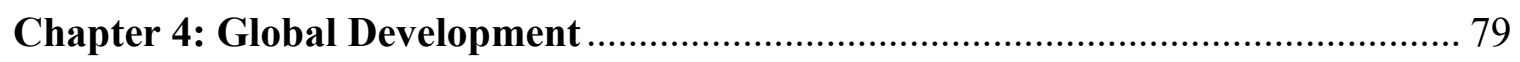

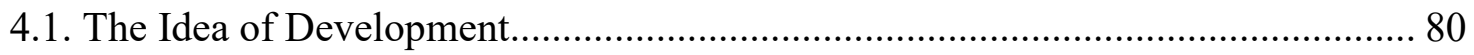

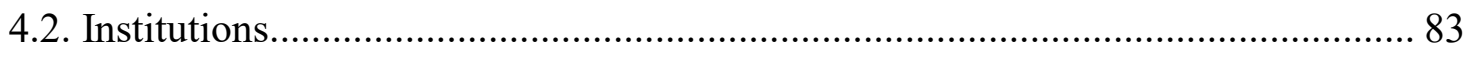

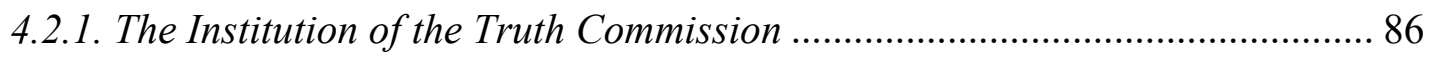

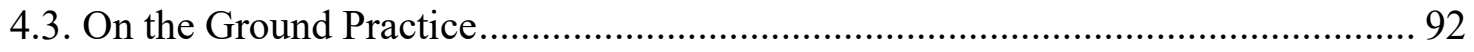

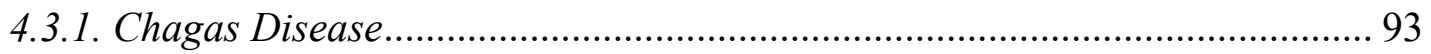

4.3.2. Tanzanian Health Care Initiatives ............................................................. 97

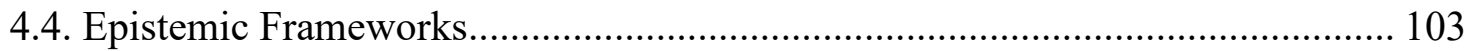

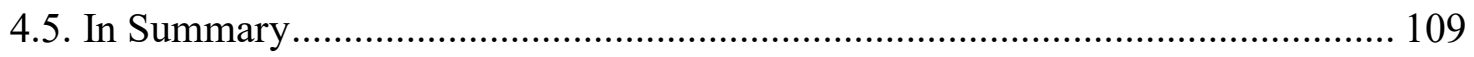

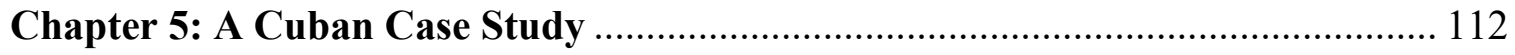

5.1. A Brief Review of Cuban History ............................................................... 116

5.2. The Epistemic Framework ....................................................................... 118

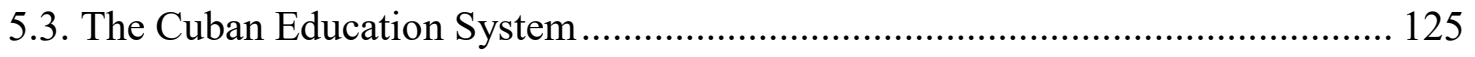

5.4. The Cuban Health Care System .................................................................. 131

5.5. Cuban Internationalism in Education and Health ......................................... 136

5.6. Concluding Remarks: A New Approach to the Global Role of Epistemic

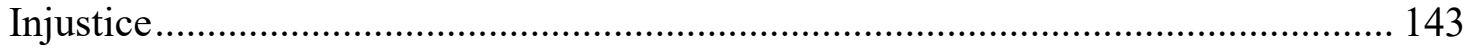

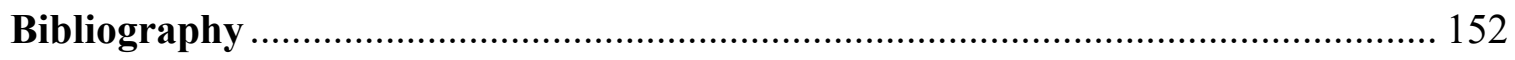




\section{Introduction}

A decade later, Miranda Fricker's 2007 book, Epistemic Injustice, has been a catalyst for new ways of thinking about the intersection between epistemology and ethics. In it, Fricker focuses on the wrongs done to someone in their capacities as a knower (Fricker $2007,20)$, wrongs which harm them in an essential aspect of their very humanity (44) while at the same time having further harms with real, practical impact (46). From her account, which focuses on two specific categories of epistemic injustice in the forms of testimonial injustice and hermeneutical injustice, a great deal of literature has been spawned which both praises the important contribution she has made (Maitra 2011, 195; Alcoff 2010, 128; Anderson 2012, 165; Dotson 2012, 25; Hookway 2010, 151; and Medina 2011, 15) and critiques her approach (these critiques will be discussed in depth throughout this thesis).

In one of those critiques, Kristie Dotson proposes viewing Fricker's account as "an account of epistemic injustice", as opposed to "the" account of epistemic injustice, opening the possibility of it being one theory among many (Dotson 2012, 41). Fricker's account has had a useful part to play in introducing the issues of epistemic injustice to the philosophical community. However, it does not always adequately grasp the complexity of what is happening in such cases. This thesis will propose another theory of epistemic injustice, one that I argue is more appropriate for use in a complex, global scale, crosscultural context such as that presented by global development.

In Chapter 1, I will present an in-depth explanation of Fricker's account of epistemic injustice, as it is outlined in Epistemic Injustice. I will detail the harms and 
wrongs of both testimonial injustice and hermeneutical injustice in order to draw out the excellent ground work that Fricker's account provides.

In Chapter 2, I will explore some of the most relevant and serious critiques of that account, to demonstrate why a different approach in needed. Specifically, I will look at critiques of Fricker's concept of "the collective hermeneutical resource", the risk of accusations of false consciousness and imposing concepts, the gaps in Fricker's account, the need for a more structural and relational approach, and the problem with using strict categorization to identify the kinds of epistemic injustice.

In Chapter 3, I will present my new account of epistemic injustice, focusing on an approach that does not rely on categories and which pays attention to relationships and to alternative resources for knowing beyond the dominant ones. I will use the example of white ignorance to focus on what the new account has to capture, and will draw on work by María Lugones and Lorraine Code to shape my new approach.

In Chapter 4, I will apply this new theory of epistemic injustice to several areas of global development: the concept of development itself, the institutions of development and the policies that tend to get endorsed, the practice of development on the ground, and the conceptual frameworks that those policies and practices draw on.

The final chapter will present a case study, looking at how epistemic injustice plays out in relation to Cuba's education and health care policies, as well as their potential contributions to global development. In particular, I will focus on the marginalization and discrediting of the epistemic framework provided in the philosophy of José Martí and Che Guevara. This focus on the philosophical background is important because it is the foundation of the Cuban revolution and all its achievements. 
My goal is to demonstrate the interconnected ways in which the devaluing, discrediting and marginalization of the experiences and knowledge of "underdeveloped" countries and peoples harms those people and prevents improvements to their well-being. I also seek to tease out the ways in which the rest of our global society also loses out on important resources when it silences or ignores these other ways of looking at and being in the world. Although within the scope of this project, I am only able to examine what the problem is and how it should be approached, the identification of epistemic injustice is the first step towards being able to prevent and mitigate it. 


\section{Chapter 1: Fricker's Account}

This chapter will provide a detailed explanation of Miranda Fricker's theory of epistemic injustice as it is presented in Epistemic Injustice: Power and the Ethics of Knowing. As a general concept, an "epistemic injustice" is an injustice that wrongs someone in their capacities as a knower $(2007,1)$. Within this general concept, Fricker divides epistemic injustice into two types, "testimonial injustice" and "hermeneutical injustice". Both types of injustice share the epistemic wrong to the harmed individual that is central to the concept of epistemic injustice. However, Fricker believes that the two differ in how they are caused and how their wrongs and harms manifest. This account has many merits, in that it successfully draws attention to the intersection of ethics and epistemology, and clearly articulates the harms that occur when prejudice and marginalization negatively impact the epistemic activities of particular social groups. However, it also has flaws, which will be discussed in Chapter 2. As such, it has the potential, I will argue, to provide an excellent background for a discussion of how harms arise in global development, but it does not tell the whole story.

\subsection{Testimonial Injustice}

The first of the two categories of epistemic injustice that Fricker discusses is "testimonial injustice". This type of epistemic injustice is found in the exchanges between individuals, where the credibility of a speaker is negatively affected by a prejudice on the part of the hearer against some aspect of the speaker's social identity. In Fricker's words, "testimonial injustice occurs when a prejudice causes a hearer to give a deflated level of credibility to a speaker's word" $(2007,1)$. 
Fricker's account of testimonial injustice begins by describing the important role that social stereotypes, defined as "widely held associations between a given social group and one or more attributes" (30), play in allowing hearers to evaluate the credibility of someone that they are speaking to (17). She claims that she is using the word stereotype "in a neutral sense", as there can be positive as well as negative stereotypes, and they can be both reliable and distorting (30). Linda Martín Alcoff has summarized Fricker's use of the neutral sense of stereotype as "mean[ing] merely something like a generalization or heuristic that can be innocuous, at least in some cases" $(2010,130)$. Reliable stereotypes allow a hearer to use a short cut in deciding whether information can be believed, freeing up mental capacity and speeding up the transfer of information (Fricker 2007, 31-32). For example, the stereotype of "the dependable family doctor", if it is a reliable generalization about family doctors, is a useful tool for shaping how we view the medical advice being given by such doctors (32).

However, if the stereotype is one that embodies a negative and distorting prejudice towards the speaker despite evidence to the contrary, then the stereotype causes "an epistemic dysfunction in the exchange" (17). Such prejudicial stereotypes are an "unreliable empirical generalization about the social group in question" (32), and as a result should not be used in determining what to believe. Fricker uses the term "identity prejudice" to denote prejudicial stereotypes against individuals resulting from their membership in a particular social group. For example, the stereotype that women cannot achieve the skill required to be successful neurosurgeons would be an identity prejudice and an unreliable stereotype. It is these prejudices, and not stereotypes more generally, that Fricker argues are at the center of testimonial injustice. Because a prejudice is "a 
judgement made or maintained without proper regard to the evidence" (33) and is, therefore, unreliable, using a stereotype based on a negative identity prejudice to determine that someone's testimony deserves little credibility is an epistemically and ethically culpable mistake.

Prejudicial stereotypes can cause both credibility excesses and credibility deficits. However, Fricker argues that only credibility deficits can cause testimonial injustice (21). According to Fricker, this is because only credibility deficits have the characteristic of harming the speaker in their capacity as a knower, making the wrong distinctly epistemic as well as ethical (20). She claims that credibility excess, on the other hand, "does not undermine, insult or otherwise withhold a proper respect for the speaker qua subject of knowledge" (21). However, critics have pointed out other ways in which credibility excess could be considered an epistemic injustice. That critique will be discussed in Chapter 2.

Regardless of whether one accepts this aspect of Fricker's account, it is important to note that the prejudices most likely to harmfully impact credibility are those that obstruct the hearer from accurately perceiving the speaker's sincerity or competence. This is because the two characteristics of sincerity and competence are necessary components in evaluating the epistemic trustworthiness of a subject (45). The unequal power relations in society, as well as oppressive social structures, make certain individuals and groups more likely to be attributed lower levels of sincerity or competence due to prejudice. With those lower levels of sincerity or competence will come a credibility deficit, and with that credibility deficit will come a testimonial injustice. 
Fricker does argue that not all credibility deficits are the result of harmful prejudice. She argues that errors in credibility judgement that are either ethically and epistemically non-culpable, or ethically innocent but epistemically culpable, do not cause testimonial injustice. To use Fricker's example, imagine an ethicist who knows that she is going to be speaking to another academic, and upon researching him online believes he is a medical doctor since his name is listed under the medical school faculty list. During their conversation, the topic of her specialization in moral fictionalism comes up as he expresses a critical view of the approach. She gives his position lower credibility on that topic than she would give someone who she believed was an ethicist. Unfortunately for her, he is in fact an ethicist, "and so her false belief about his professional identity has put him in a credibility deficit" (22). This credibility deficit could conceivably be described as stemming from an identity prejudice, because the ethicist's beliefs about the other academic's identity lead her to incorrectly reduce the credibility she attributed to his critical view. However, if he really had been just a doctor rather than an ethicist as well, the credibility judgement she made would likely not have been a deficit but rather an accurate evaluation of his knowledge on the topic of moral fictionalism. For Fricker, this seems to be a case of an innocent error on the part of the ethicist and not a testimonial injustice.

In Fricker's example, the ethicist is not prejudiced against non-ethicists or doctors. Instead, she has reasonable doubts about their knowledge of the details of ethical debates in academia. There would be not testimonial injustice because the wrong of a testimonial injustice stems from an "ethical poison in the judgement of the hearer" that is caused by prejudice (22). Without that prejudice, the ethical wrong of a testimonial 
injustice is absent, as it seems to be absent in this example. According to Fricker, innocent error, not testimonial injustice, would also be at play if the perpetrator had been epistemically culpable for the error because of a careless web search (22), or because she had simply assumed he wasn't an ethicist without any evidence. This seems to me to be a reasonable evaluation of cases where a credibility judgement is incorrect due to a flaw in the information a hearer has rather than a prejudice against the social identity of the speaker.

Fricker also recognizes that sometimes a prejudicial mistake in assigning credibility will be the result of "epistemic bad luck" (33), where it is through no fault of the individual that they hold the prejudice in question. Such epistemic bad luck will usually be the circumstantial result of a historical context where it would take "a very exceptional epistemic character to overcome these prejudices" (33). Fricker draws on the example of Herbert Greenleaf from The Talented Mr. Ripley to demonstrate this point. Greenleaf is a man in the 1950s who distrusts his son's fiancé, Marge Sherwood, in her continual insistence that her fiancé's friend, Tom Ripley, killed her fiancé. Greenleaf's disbelief of Sherwood is based on his prejudice that women tend to be more emotional and rely on their intuition rather than reason, particularly when it comes to men they love. This was a prevalent prejudice of the time. Unfortunately for Greenleaf, Sherwood's accusation of Ripley is entirely reasonable and evidence based (100). However, it is bad luck contingent on his historical context that leads to Greenleaf's epistemic resources being inadequate, which leads him to an incorrect and unjust conclusion.

According to Fricker, because of this bad luck Greenleaf is not doing anything ethically blameworthy by using the stereotypes and prejudices of his time in his 
testimonial exchange. Instead, he is failing to act in an ethically exceptional way. This failure carries less responsibility on his part than full ethical blameworthiness, although Fricker thinks it is still reasonable for those who are reading about Greenleaf's dismissal of Sherwood to "feel some sort of qualified moral resentment towards him" (104). She argues that this "resentment of disappointment" stems from a feeling on the part of the reader that "he could have done better" ethically (104). It was possible, though unlikely, that he could have done something ethically exceptional with the conceptual resources he had in order to avoid the harm that he does to Sherwood. However, Fricker also argues that the resentment of disappointment points to a lesser degree of responsibility in the person it is aimed at than "the resentment of blame" (104). This lesser responsibility should be attributed to Greenleaf because "if a hearer cannot be blamed for the grounds of [his] flawed credibility judgement, then [he] cannot be blamed for the harm that may result " (42). Although it is understandable that Fricker wants to accommodate the possibility that people in some circumstances do not have the resources to avoid committing an epistemic injustice, this exemption of blameworthiness does not seem to provide a satisfying account of who is responsible for the epistemic injustice that still occurs. This is a troubling aspect of Fricker's approach, one that Ishani Maitra critiques and which will be discussed in section 2.2.2.

Even when an ethically culpable prejudice is present, the type of prejudice that it is changes the type of testimonial injustice that is experienced. Fricker identifies the subcategory of "incidental testimonial injustice", where the prejudice in question causes credibility deficits in a "highly localized" manner that restricts its impact to only epistemic injustice, rather than also leaving the subject open to legal, political, economic, 
and social injustices (27). She presents the example of a scientific journal whose panel of referees have "a dogmatic prejudice against a certain research method". Anyone submitting a paper to this panel that presents a hypothesis based on said research method will receive a reduced level of credibility because of using that method (27). Although this generates a genuine testimonial injustice, because the credibility deficit is caused by a prejudice against the author due to their method and not the actual content of the study, according to Fricker the impact of that prejudice is isolated to that particular exchange. Fricker argues that these incidental cases do not negatively impact the power relations in society as severely as those that are more structural, and as a result they are less worrying to those concerned about oppression and marginalization.

I would agree with Fricker that this example is a case of testimonial injustice. Methodology can be considered an aspect of the author's "social identity" since it reflects the epistemic framework the author is using, which will usually be embedded in their culture, beliefs, values, and socialization. However, I do not think that Fricker's example of a dogmatic prejudice against a certain research method reflects a case where the credibility deficit is highly localized and thus incidental. I will argue that prejudice against a specific research method, field of study, or approach in academia could reflect the hermeneutical marginalization and discrediting of an entire epistemic framework in way that does negatively impact the power relations in society and which could contribute to the systematic oppression of a particular social group. In Chapters 4 and 5 I will discuss examples of how prejudice against certain epistemic frameworks causes an epistemic injustice that needs to be addressed. José Medina also provides a critique of Fricker's account of incidental testimonial injustices, particularly as they relate to 
credibility excess and to larger patterns of injustice. His critique will be discussed in section 2.4 .

For Fricker, the far more concerning sub-category of testimonial injustice is "systematic testimonial injustice". The systematic case is the result of a type of prejudice that "renders one susceptible not only to testimonial injustice but to a gamut of different injustices" (27). Fricker identifies it specifically as stemming from an identity prejudice that is based on membership in a particular social group (27). This kind of identity prejudice causes not only testimonial injustice in one specific knowledge exchange, but will usually affect all areas of the individual's life. As a result, Fricker identifies this as a systematic "identity prejudicial credibility deficit" (28), and calls it "the central case of testimonial injustice" (28). This central case is the most concerning for theorists who wish to counter injustice because the credibility deficits in these cases will follow the power structures of society that track social identity. Because a systematic identity prejudice results in a person facing not only epistemic injustice, but usually also economic injustice, political injustice, social injustice, and many other forms of injustice, it is the more severe type of testimonial injustice. It should be noted that although this is the central case of testimonial injustice, Fricker does emphasize that there are other cases of testimonial injustice that are not systematic but that still count as testimonial injustices and "may be utterly disastrous for the subject" (29).

Fricker uses Harper Lee's To Kill a Mockingbird as the primary example of systematic testimonial injustice. I would argue that Sherwood's experience described earlier is also an example, even though Fricker identifies it as a case of epistemic bad luck, because the identity prejudice and the harms it causes Sherwood follow her through 
all aspects of her life. In To Kill a Mockingbird Tom Robinson, a young black man, is accused of beating and raping a white girl, Mayella Ewell. Atticus Finch, Robinson's white lawyer, proves beyond a reasonable doubt that there is no way that Robinson could have done what he is accused of doing, because he was unable to use his left arm. However, the all-white jury delivers a guilty verdict. The testimonial injustice occurs when Robinson's own account of the incident, which includes him feeling sorry for Ewell (24) and her trying to kiss him (23), is assumed to be false simply because he is a black man. The jury's negative and distorting prejudices that all black people lie (23), that there is no feasible reason that he could feel sorry for a white person no matter how poor she is (24), and that black men are sexually aggressive (25) together destroy Robinson's credibility. The jury was from the beginning almost certainly disposed to not believe he is telling the truth, regardless of the evidence. However, Finch is subject to the same historical context and social influences as the jury yet manages to grant Robinson the credibility that his testimony is due. As a result, one cannot attribute epistemic bad luck to the jury members as a defense for the testimonial injustice they commit. The jury is both ethically and epistemically culpable for the credibility deficit, and also culpable for the secondary practical harms that result from this testimonial injustice. These secondary harms include Robinson's conviction, incarceration, and eventually his death (26). The identity prejudices in question also tracked Robinson through many other areas of his life. Moreover, his receiving a guilty verdict, even counter to evidence, contributed to the perpetuation of oppression and marginalization of black men in that era.

For Fricker, Robinson's experience is an example of the central case of systematic testimonial injustice, where a hearer gives the speaker's testimony less credibility than it 
is due because of an identity prejudice against them. In this case, the person who is discredited does give their testimony, and the hearer does engage with that testimony. It is in giving them lower credibility than they deserve that the harm and the wrong occur. However, Fricker also discusses two other subtypes of systematic testimonial injustice, where the testimony of the person who is discredited is not even heard. These are instances of silencing.

The first form of silencing is pre-emptive testimonial injustice, where members of social groups who are the subjects of identity prejudice "tend simply not to be asked for information in the first place" (130). In this case, the silencing occurs before any potential information exchange can happen, stopping it before it can start and allowing the structural workings of identity power to control what epistemic contributions are publically expressed (130). The residential school system used historically in Canada to "educate" and in the process assimilate indigenous children to European culture presents an example of pre-emptive silencing. The Government of Canada, in coordination with many of the churches across the country, put in place this program for the forced assimilation of indigenous peoples. It started in the 1830s, and lasted all the way until 1998 when the last school closed (Truth and Reconciliation Commission 2015, 70). Indigenous children were removed from their parents without the parents' consent or consultation, with the intention that the system would "continue until there is not a single Indian in Canada that has not been absorbed into the body politic, and there is no Indian question" (Truth and Reconciliation Commission 2015, 54). At the heart of the policy was a deep-seated prejudice against indigenous peoples, their culture, and their forms of knowledge. It was assumed that indigenous people had nothing legitimate or valuable to 
contribute to what would eventually become Canada, and that what was "best" for the children was to teach them Western values and practices in order to eliminate their traditional culture and assimilate them into the culture of their colonizers.

The result was a disastrous system that failed to give the children a "good education" even by European standards. Entire cultures have been lost or are on the verge of disappearing because of that system, and the extent of the abuses perpetrated against survivors while in residential schools are still coming to light, as are their intergenerational effects. Under the residential school system, pre-emptive silencing occurred when parents in indigenous communities were not consulted or seen as authoritative about what would be best for their children, and when stories and reports about the problems with the system were ignored. It also happened at a broader level, when the separation of children from their languages and their communities led to the elimination of certain cultures and the perspective and knowledge they could provide. The silencing of these perspectives was not only a testimonial injustice, but also an example of the second type of epistemic injustice that Fricker discusses: hermeneutical injustice. This second type of epistemic injustice will be discussed further in the next section of this chapter. This example goes beyond Fricker's account, showing how silencing happens not just to individuals but to entire cultures and their epistemic frameworks. I will discuss both the example of Canadian residential schools and the importance of epistemic injustices against epistemic frameworks in later chapters.

Silencing is not necessarily pre-emptive, however. Another method of silencing that Fricker identifies as a systematic testimonial injustice occurs when the person who is silenced speaks, and may even be asked for information, but their testimony is not heard. 
Catharine MacKinnon describes an example of this type of silencing, arguing that the objectification of women can lead to their testimony not being heard as testimony, particularly by men, even when it is expressed. This is because she is attributed with so little agency and subjectivity that the man fails to register that she is presenting information when she speaks (Fricker 2007, 139). As a result, there is no engagement from the hearer, no reciprocity, and no uptake (140), leading to her not being heard because she is objectified. This is not pre-emptive, because testimony is given, but it is also not the same as the central case, because the testimony is not heard so it cannot be given reduced credibility. It is given no credibility at all. Both cases of silencing amount to extreme testimonial injustice, as the identity prejudicial credibility deficit is so pervasive that it doesn't matter what the person who is silenced says, they won't even be heard let alone believed. It also serves to reinforce marginalization and oppression, as it excludes the person who is silenced from important epistemic activities that can help to reshape the power structures of the society.

Jill Stauffer discusses another form of silencing in her account of ethical loneliness. She calls ethical loneliness "the injustice of not being heard", and describes it as "the isolation one feels when one, as a violated person or as one member of a persecuted group, has been abandoned by humanity, or by those who have power over one's life's possibilities" (Stauffer 2015, 1). It happens when someone comes out of a situation of injustice "only to find that the surrounding world will not listen to or cannot properly hear their testimony" (1). The result is a compounding of the injustice they have already experienced, where not only has the subject undergone the terrible harms and wrongs of the original injustice, but they then undergo the experience of "being 
abandoned by those who have the power to help" (5). Although ethical loneliness results in part from the types of silencing that Fricker outlines, it also contains an additional element of expectation towards those who are not hearing. If ethical loneliness is being abandoned by those with the power to help, then there is an implied relationship between the person being silenced and the person who is abandoning them. The relationships of power at stake in Stauffer's account, as well as in the Canadian residential school system, are set in a broader context that is not examined by Fricker, and will be discussed in section 4.2.1.

As mentioned previously, the primary harm of all types of testimonial injustice, whether incidental or systematic, is the harm that is done by wronging the speaker in their capacity as a knower. This "is a form of the essential harm that is definitive of epistemic injustice in the broad" (Fricker 2007, 44), and differentiates it from other forms of injustice. According to Fricker, "to be wronged in one's capacity as a knower is to be wronged in a capacity essential to human value" (44) because "the subject is wronged in her capacity as a giver of knowledge" which is "one side of that many-sided capacity so significant in human beings: namely, the capacity for reason" (44). This means that "even its least harmful instances [have] a symbolic power that adds a layer of harm of its own: the epistemic wrong bears a social meaning to the effect that the subject is less than fully human" (44). When a person's capacity as a knower and their capacity for reason is brought into question, so is their agency and their ability to engage in any kind of productive epistemic activity.

This primary harm can then lead to secondary harms in both the practical (46) and epistemic dimensions (48) of the person's life, influencing all aspects of their personhood 
and even undermining their sense of self (53). It will also reinforce the power relations of oppression and marginalization that cause the prejudice in the first place. These secondary harms will be particularly pernicious in cases of systematic testimonial injustice, because of the depth and breadth of their influence. In Chapter 4, I will expand analysis of the harms of epistemic injustice further, through its application to the context of global development.

\subsection{Hermeneutical Injustice}

The second category of epistemic injustice that Fricker identifies is hermeneutical injustice. Unfortunately, in Epistemic Injustice, she provides much less detail about this type than she provides about testimonial injustice. However, this is at least partially because the two types of epistemic injustice share a great deal in common when it comes to their epistemic character, their genealogy, and their sub-categorization into either incidental or systematic cases. It is also because Fricker thinks that testimonial injustice is "genealogically the most basic" form of epistemic injustice (Fricker 2010, 174), because testimonial knowledge is "ubiquitous and basic in human knowledge" (176). As a result, her approach builds an understanding of epistemic injustice primarily based on testimonial injustice, with hermeneutical injustice in the background. In Chapter 2, I will discuss critiques that draw attention to the interconnected relationship that exists between hermeneutical and testimonial injustice, which will bring into question the apparent primacy of testimonial injustice.

According to Fricker's account, hermeneutical injustice occurs in the background of epistemic activities and exchanges. It is caused by a gap in shared tools of social interpretation "where it is no accident that the cognitive disadvantage created by this gap 
impinges unequally on different social groups" because these groups "participate unequally in the practices through which social meanings are generated" (Fricker 2007, 6). This comes to light in the discussion of Canadian residential schools and of Stauffer in the previous section as well.

This gap in shared resources has an impact not only on the content but also the form of what can be said and understood (160). This is due to unequal relations of power in society, which lead to some social groups being prevented from participating on equal terms with more privileged groups in such meaning generating practices (152). These power relations are also evident in testimonial injustice, and lend authority and credibility to some speakers while undermining that of others, such as Ripley having more credibility with Greenleaf than Sherwood does in the example of the Talented Mr. Ripley, and Ewell's father having more credibility with the jury than Robinson does in To Kill a Mockingbird. Men, and white people, automatically have more credibility because of the greater power they enjoy. Fricker states that "most obvious among such practices are those sustained by professions such as journalism, politics, academia and law" (152), where a lack of participation from disadvantaged groups leads to privileged groups dominating the generation of the kinds of meanings that are produced in those fields and then used by the rest of society. She calls this unequal hermeneutical participation (152), and argues that this unequal participation leads to hermeneutical marginalization (153) and results in a structurally prejudiced collective hermeneutical resource (155). In other words, because of their unequal participation in the formation of meaning and the development of shared tools of understanding, some groups are subordinated and 
excluded from a practice that would be valuable for them to have access to in their own attempts to generate meaning and be understood by the society at large.

The result is that the society's tools for understanding are structured in a way that discriminates against some members of the society because of their suspected membership in particular social groups. Fricker calls this "structural identity prejudice". She also argues that because of its occurrence in the background structures of epistemic exchanges (154), there is no direct perpetrator of hermeneutical injustice (159), although sometimes there may be some party or group that should be held responsible for the hermeneutical marginalization. This reflects the individualism of her account, and will be critiqued in later chapters.

Fricker cites the experience of Carmita Wood to exemplify the core, systematic case of hermeneutical injustice. Wood was a woman working as an administrative assistant in Cornell's Department of Nuclear Physics in the 1970s, and experienced unwanted sexual gestures and touches from a distinguished male professor in the department. The stress of trying to avoid the man and trying to deal with the situation led to her experiencing chronic back and neck pain, as well as other physical symptoms. She tried to get a transfer to another department, and when that failed she quit her job. However, when she applied for unemployment insurance and was asked why she had left her job, she "was at a loss to describe the hateful episodes. She was ashamed and embarrassed" and so simply cited personal reasons (150). In effect, she was silenced because of a testimonial injustice that prevented her experience being added to the collective hermeneutical resource and as a result, she did not get unemployment insurance. It was only later, after Wood discussed her experience with other women and 
found that it was something many had experienced but could not put a name to, that sexual harassment as a distinct wrong with significant harms began to be recognized.

The collective resources that were available to her when she was dealing with the university led to others telling Wood that she should be flattered to have the attention of someone so distinguished, that it was just harmless flirting, and that she was overreacting and couldn't take a joke. This explanation failed to recognize the harms that Wood was experiencing. The collective meanings of what is appropriate in the workplace environment of a university had been developed almost entirely by men, so a gap in hermeneutical resources existed around what Wood needed to be able to communicate about the harms done to her. That structural identity prejudice not only stopped Wood from being able to share her experience in a manner intelligible to those it was in her interest to communicate it to (resulting in her not being able to access employment insurance) but also resulted in her further exclusion from certain roles that could contribute to meaning generation, such as working at a university. Critiques by Gaile Pohlhaus Jr. and Medina also pull out the importance of paying attention to the relationships that Wood develops with other women who have a similar experience, which allows collective action and the formation of alternative understandings. This will be discussed further in Chapter 2.

Although the harasser's actions caused a variety of harms to Wood, according to Fricker the perpetrator of the sexual harassment is not the perpetrator of the hermeneutical injustice. Fricker argues that while he did something wrong and harmful, the gap in understanding about why his actions are wrong (a gap that also leads to other people to not adequately acknowledging Wood's experience) is not his fault but is instead 
a result of an injustice in the structures of society with no identifiable perpetrator. However, Fricker's analysis ignores the role that relationships of power and authority play in making an individual such as Wood's harasser, or Greenleaf, responsible for the harms that epistemic injustice causes even if the structures of society make it more difficult for them to recognize the wrong they are committing. The structural injustice that causes the hermeneutical injustice is the marginalization of women and their experiences to the point where their knowledge and experiences can't be understood by those who don't share them, and men such as Wood's harasser help to hold these structural injustices in place. It is also evident here that a testimonial injustice is at play, where Wood is silenced or at the very least discredited by those to whom she tries to tell her story. The structural injustice that causes the hermeneutical injustice also causes the testimonial injustices, and it is clear yet again that the two types of epistemic injustice are deeply connected. In contrast to Fricker, my analysis in Chapters 2 and 3 will demonstrate how necessary this interconnection is for understanding epistemic injustice as a whole.

As with the category of testimonial injustice, according to Fricker, not all cases of hermeneutical injustice are systematic. While systematic hermeneutical injustice, like systematic testimonial injustice, tracks the subject through social activities beyond just the epistemic, there can also be cases of "incidental hermeneutical injustice". These are similar to the case of incidental testimonial injustice, where the marginalization is momentary and not embedded in a structural inequality of power that tracks someone throughout their life (156). Also like systematic testimonial injustice, systematic 
hermeneutical injustice is seen by Fricker to be of more concern to her theory than the incidental cases of hermeneutical injustice.

The primary harm of hermeneutical injustice is that the marginalized individual or group "is rendered unable to make communicatively intelligible something which it is particularly in his or her interests to be able to render intelligible" (162). This is simply a different manifestation of the primary harm of epistemic injustice more generally, "being wronged in a capacity essential to human value" (44) which is specifically their "capacity as a knower" (44). Fricker calls this a situated hermeneutical inequality. In such situations, it is in the subject's interest to communicate something so that it can be heard and understood, but they are unable to do so because their experiences are excluded from the understanding of many of their interlocutors. According to Fricker, the background conditions of hermeneutical injustice will frequently be present without the actual harm, and "the moment of hermeneutical injustice comes only when the background condition is realized in a more or less doomed attempt on the part of the subject to render an experience intelligible, either to herself or to an interlocutor" (159). Fricker is likely right that attention will not be drawn to the hermeneutical marginalization and the harms that it can cause unless someone tries to communicate an experience that cannot be understood. However, this serves to further throw into doubt the possibility of separating instances of hermeneutical injustice from instances of testimonial injustice. Paying attention to the relationships between the categories, and between individuals and the groups that they are members of, will throw some of the assumptions made by Fricker about what hermeneutical resources individuals have access to into doubt. How this can be done will be discussed in Chapters 2 and 3. 
The primary harm of hermeneutical injustice, like that of testimonial injustice, also leads to further secondary harms. An experience that is not understood by society at large cannot be dealt with appropriately, particularly if it is the result of systematic prejudice, inequality, and oppression. Furthermore, it will have severe implications for the negatively impacted person's sense of self and confidence in their own knowledge, because when "you seem to be the only one to feel the dissonance between received understanding and your own intimate sense of a given experience, it tends to knock your faith in your own ability to make sense of the world" (163). As a result, hermeneutical injustice can do deep seated damage to the very selves of those who are marginalized and oppressed.

\subsection{In Summary}

To this point, I have provided a thorough summary of Fricker's account of epistemic injustice as it is presented in Epistemic Injustice. Although it has many strengths, and has contributed a great deal to the conversation about what happens when someone's social identity impacts how their knowledge is perceived by others, there is still a great deal more that can be done. The rest of this project will try to shape a theory of epistemic injustice that is more holistic, remedying many of the shortcomings in Fricker's account. I want to build a theory of epistemic injustice that can adequately account for the complexities of cross-cultural, multi-dimensional interactions such as those that arise in the setting of global development. In the next chapter, I will examine many of the criticisms that have been raised against Fricker's account, with the intention of formulating an approach to epistemic injustice that is appropriate for global development contexts. This chapter has begun to show how the discussion of epistemic injustice can be 
moved into a broader context than what Fricker provides, through the examples of Canadian residential schools and from Stauffer, and what follows will take it even further. 


\section{Chapter 2: Critiques of Fricker's Account}

In this chapter, I will examine those critiques of Miranda Fricker's approach that are the most serious and relevant to my project. The first set will focus on criticisms of the category of hermeneutical injustice from José Medina, Rebecca Mason and Gaile Pohlhaus Jr. The second will look at various types of epistemic activity and aspects of epistemic injustice that Fricker's account misses, focusing on arguments by Christopher Hookway, Medina, and Ishani Maitra. The third will draw on Elisabeth Anderson, Medina, Linda Martín Alcoff, and Sarah Lucia Hoagland to identify accounts or approaches that could better shed light on what is happening in cases of epistemic injustice. The last will critique the process of categorization itself. Taken together, these critiques will help to draw out what needs to be done in order to create an account of epistemic injustice that more adequately encompasses the complexity of the phenomenon that Fricker has identified. That is what I plan to do in Chapter 3.

\subsection{Critiques of Hermeneutical Injustice}

Many of the most relevant critiques raise problems for epistemic injustice as a whole. There are, however, several critiques that are particular to how the category of hermeneutical injustice is defined and how theorists should understand the harms and wrongs caused by certain groups being marginalized from the production of shared knowledge and hermeneutical resources. Because what Fricker describes as hermeneutical injustice forms the background for other unjust encounters, it is important to clarify exactly what is occurring in these situations in order to ensure that the theory does not itself perpetuate epistemic injustice. 


\subsubsection{The Problem with the "Collective Hermeneutical Resource"}

The first problem with Fricker's account of hermeneutical injustice is one that Pohlhaus, Medina, and Mason all identify. An essential part of her definition of hermeneutical injustice involves a "gap" in what she calls the "collective hermeneutical resource" (Fricker 2007, 6). She argues that marginalization from that "collective hermeneutical resource" leads to situations where "non-dominant groups fail to understand their social experiences" (Mason 2011, 299), which Fricker takes to be a hermeneutical injustice. All three critics argue that in this definition, Fricker does not account for the hermeneutical resources that are developed inside of marginalized communities (Mason 2011, 299; Medina 2012a, 207; Pohlhaus 2012, 724), and as a result Fricker "conflates collective with dominant hermeneutical resource" (Mason 2011, 305). In doing so, she ignores how the members of marginalized communities can use more localized, non-dominant hermeneutical resources developed among people who share their experiences in order to understand those experiences (Mason 2011, 295; Medina 2012a, 210; Pohlhaus 2012, 724). I will refer to these as alternative hermeneutical resources.

Alternative hermeneutical resources are those shared resources that people draw on for understandings that are specific to a particular social group outside of the dominant one(s). Women share hermeneutical resources with regard to experiences that are particular to women, such as facing sexism when trying advance in their careers. These experiences are not shared by men, who are the dominant social group, and as a result will likely not be incorporated into the dominant hermeneutical resource (the term I will use in place of Fricker's "collective hermeneutical resource"). However, the alternative hermeneutical resources available to women and shared with other women will mean that 
they can still understand their experiences and communicate them to people who share them, even if they cannot successfully communicate them to men who do not because they are silenced, ignored or discredited. These alternative hermeneutical resources arise in other marginalized communities as well, such as racial minorities, sexual minorities, gender minorities, lower economic classes, etc. The presence of these alternative understandings must be recognized in a theory of epistemic injustice, because they are a source of agency and resistance for the marginalized and oppressed social groups. They are also a source of hope and potential for overcoming the hermeneutical marginalization of such groups from the dominant hermeneutical resource.

The presence of alternative hermeneutical resources draws attention to an incomplete aspect of Fricker's approach, where the parameters of hermeneutical injustice need to be clarified. In her account, it seems that when there is a hermeneutical injustice, no one can understand the marginalized person's experience. However, recognizing alternative hermeneutical resources reveals that a gap in the dominant hermeneutical resource does not necessarily mean that a person who is marginalized from the dominant hermeneutical community is unable to understand their own experiences, or that other members of their marginalized community won't recognize the experience. Part of the problem is that Fricker assumes the perspective of the dominant group, only taking into consideration the hermeneutical resources available to the powerful. This dominant perspective is also what leads her to excuse Herbert Greenleaf from blame and instead call the epistemic injustice he does to Marge Sherwood "epistemic bad luck". The problems with Fricker's use of epistemic bad luck will be discussed further in section 2.2.2. The existence of alternative hermeneutical resources needs to be addressed if those 
harmed by hermeneutical injustice are to be fully respected as epistemic agents with their own ways of knowing and understanding the world. In order for theorists and practitioners to avoid re-entrenching epistemic injustice, oppression, and marginalization, those harmed by injustice need to be acknowledged as having an active role in and knowledge of their own beliefs, values, and experiences. Otherwise, they are treated as less than fully human agents.

However, whether the availability of alternative hermeneutical resources mitigates the harms of hermeneutical injustice is a matter of debate among critics. While Mason claims that "because the marginalized have other resources to draw on to interpret their experiences, gaps in the dominant hermeneutical resource don't necessarily lead to hermeneutical injustice" (Mason 2011, 300), Medina argues that "the hermeneutical injustice continues even after the lack of self-understanding disappears" (Medina 2012a, 207). The difference here seems to depend on how one interprets Fricker's statement that hermeneutical injustice occurs when "a gap in collective interpretive resources puts someone at an unfair disadvantage when it comes to making sense of their social experiences" (Fricker 2007, 1, my emphasis). It is unclear who is supposed to be "making sense of their social experiences". Either she means that hermeneutical injustice only occurs when non-dominant social groups do not know their own experience, or she means that it also occurs when dominant groups do not know the experiences of nondominant groups (Mason 2011, 295). If hermeneutical injustice always and necessarily includes the marginalized not knowing or understanding their own experience, then Mason is correct that when non-dominant groups use alternative hermeneutical resources to understand their experiences the hermeneutical injustice is eliminated. However, if all 
that hermeneutical injustice requires is the dominant hermeneutical resource preventing someone (one's self, members of one's group, or others who don't share the experience) from making sense of an experience due to hermeneutical marginalization, then Medina is right that hermeneutical injustice occurs even when alternative hermeneutical resources fill the gap in the "collective hermeneutical resource".

I find Medina's interpretation more persuasive. For the concept of hermeneutical injustice to have the ethical and political weight that Fricker appears to want, it is necessary for the hermeneutical injustice to be present regardless of whether the person whose experience has been marginalized understands their experience. So long as the hermeneutical marginalization of the victim's relevant social group prevents someone from understanding an experience that it is in the victim's interest to have them understand, a hermeneutical injustice is present. If a hermeneutical injustice disappears as soon as the victim has some resources to understand their own experience, then the urgency of the harms stemming from hermeneutical marginalization of vulnerable groups from the dominant hermeneutical resource would seem to be greatly reduced. However, I argue that urgency should remain, even if an alternative hermeneutical resource has filled the gap for the victim and those who share their experience. This is because even if the victim understands their experience, there is still hermeneutical marginalization taking place that has important implications for their interactions with dominant groups and other marginalized groups. The dominant hermeneutical resources of a society are almost always created by the most powerful and influential groups in that society, and the unequal power relations that manifest in what hermeneutical resources are available to whom in the society will also manifest in other harms and injustices. Identifying whose 
understanding of an experience matters in hermeneutical injustice is critical for recognizing all the harms that it causes and why it needs to be addressed.

Laura Beeby expresses concern that if the terms "dominant hermeneutical resources" and "alternative hermeneutical resources" are used, rather than "collective hermeneutical resource", it will lead to some members of the dominant group feeling less responsible for ensuring no other groups are marginalized $(2012 b, 13)$. She worries that this would also lead to a lack of collective responsibility on the part of the society more broadly for the oppression that results from that marginalization. However, I think Medina's approach maintains the urgency of addressing hermeneutical marginalization in the dominant hermeneutical resource, while at the same time recognizing the agency of marginalized groups and individuals through their alternative hermeneutical resources.

On Medina's account, when marginalization results in someone being unable to intelligibly communicate their experiences to others, especially those who have the power to change the oppressive and unequal structures of society, then even when non-dominant hermeneutical resources are brought into the picture the severity of the harms of hermeneutical injustice remain. They are still not being heard by those in power, they are still being silenced and dismissed. Medina's contribution is important because an epistemically just account of hermeneutical injustice needs to recognize the ability of marginalized people to understand their experiences and to fight against their marginalization and oppression. Recognizing alternative hermeneutical resources in this way helps to ensure this agency is emphasized, while maintaining the urgency that Beeby is concerned about. That agency must be recognized, as treating people in positions of 
oppression, marginalization or deprivation as victims without the ability to fight back will only serve to re-entrench the power structures that lead to them being dominated.

Those drawing on these alternative hermeneutical resources may in fact have an epistemic advantage over those who only have access to the dominant hermeneutical resource. Often, marginalized peoples may understand more than those in dominant groups about how the world is structured, particularly about how structures of power and domination shape the world and lead to injustice. Pohlhaus discusses the epistemic significance of a knower's situatedness, particularly when they are marginally situated. She identifies a knower's situatedness as "the situations in which the knower finds herself repeatedly over time due to the social relations that position her in the world" (Pohlhaus 2012, 717). This position in the social world shapes the hermeneutical resources that one has access to, and as a result each knower "develops particular habits of attention that may attune the knower to others' habits of attention or not, depending on one's social vulnerability” (717). Pohlhaus argues that "a marginally situated knower is more likely than not to find gaps in the predominantly held epistemic resource for making sense of what is noticeable to her in view of her situatedness" (719). This is because those who are marginalized are more likely to see how inadequate our epistemic resources are for making sense of aspects of the world as they experience it (720). In other words, because gaps in the dominant hermeneutical resource are more likely to harm marginalized individuals, they have an epistemic advantage when it comes to noticing those gaps. Alternative hermeneutical resources help in both noticing those gaps and in mitigating the harms those gaps create in the lives of marginalized individuals or group members. 


\subsubsection{False Consciousness and the Problem with Imposing Concepts}

Another problem with Fricker's concept of hermeneutical injustice becomes evident when one considers how it seems to assume that oppressed groups or individuals who are victims of hermeneutical injustice suffer from a kind of false consciousness. False consciousness is a term originating from correspondence between Friedrich Engels and Karl Marx, indicating when someone faces a situation where "the real motives impelling him remain unknown to him" $(1978,766)$. If Fricker is correct that when there is a gap in the "collective hermeneutical resource", the marginalized person will be unable to understand their own experience, then there is an implication that they are experiencing false consciousness as part of the hermeneutical injustice caused by their hermeneutical marginalization. This attribution of false consciousness to oppressed groups is problematic if it entails the assumption that someone does not understand their own motives. This is because such an assumption runs the risk of taking away the agency of individuals to whom false consciousness is attributed, and undermining the validity of their beliefs and actions in unacceptable ways.

Although there is some truth to the idea that individuals may be blinded to their own oppression and the ways it impacts their beliefs and actions because of the expectations and understandings that they have internalized from their society, it is harmful to assume that individuals of an oppressed group do not understand their own experiences and motives. Assuming that false consciousness is always present in cases of hermeneutical marginalization from the dominant hermeneutical resource can very easily lead to testimonial injustice against the marginalized, through the assumption that their false consciousness makes their testimony about their experiences less credible. It can 
also lead to denying the agency of such individuals. This is why it is particularly important to acknowledge the presence of alternative hermeneutical resources when discussing hermeneutical injustice. Without recognition of the other ways beyond the dominant ones that people can understand their experience, the perspective that the dominant hermeneutical resource is the only correct way to understand the world and to judge knowledge is reinforced. Recognizing alternative hermeneutical resources reduces the risk that those who are harmed by hermeneutical injustice will be attributed with false consciousness and reduced agency.

Related to the concern about attributing false consciousness to the oppressed is the possibility that trying to counter hermeneutical injustice could lead to imposing concepts in situations where they may not be appropriate. A concept that has been developed in one culture and context to identify or address a particular experience may not be adequate for understanding or expressing how a different culture and context impact on the meaning of the same or a similar experience. When pre-existing concepts or methods from other contexts are used to attempt to fill the gap caused by the unequal hermeneutical participation and hermeneutical marginalization of some groups, there is a risk of the introduced concept being inappropriate or incorrect, which could lead to it reinforcing the hermeneutical injustice through providing new but equally inaccurate meanings. This is a large part of why epistemic injustice is such a risk in contexts of global development, and this risk will be discussed further in Chapters 4 and 5.

Kristie Dotson points out how Fricker's own account risks doing something similar. She argues that Fricker "inadvertently perpetuates epistemic oppression by utilizing a closed conceptual structure to identity epistemic injustice" (Dotson 2012, 25), 
specifically pointing to how Fricker's "framing of epistemic bad luck as an antithesis to epistemic injustice conceptually forecloses the possibility of other forms of epistemic injustice" (25). In other words, the carefully defined concepts and closed categorizes often paradigmatic to philosophical discussion may not be appropriate for a discussion about the need to pay more attention to marginalized epistemologies, especially since it excludes cases and in doing so furthers the epistemic injustice against them. The problem with Fricker's use of categorization will be discussed in more detail in section 2.4.

Another, related, concern is that Fricker's account puts too much focus on naming experiences to fill the "gaps" (Alcoff 2010, 136; Medina 2012a, 208; and Mason 2011, 298). Naming can be a very useful tool against oppression and marginalization, and in situations where injustice has been ignored, naming that injustice can be a powerful form of acknowledgement. For example, naming those who lived through the experience of Canadian residential schools as survivors, rather than as "former students" or even as victims, has played an extremely important role in recognizing the depths of the suffering and injustice that occurred under that system. The label used to fill gaps in the hermeneutical resources does matter.

However, too much focus on naming and finding the right label in attempts to counter hermeneutical injustice can prevent people from considering the possibility that some experiences can be understood even if they are not named, particularly within alternative hermeneutical resources. Focusing on eliminating hermeneutical injustice by creating labels like "sexual harassment", which easily encapsulates a concept that can then be applied to various contexts, could lead to overlooking a group's understanding of experiences that may already be present but isn't named. Even in the case of sexual 
harassment, the experience, its harms, and its wrong were all understood even before it had the label of "sexual harassment".

Naming can also be used by those in power to falsely claim that they now understand an injustice, even if they do not understand the experience of that injustice. When these unnamed understandings are ignored, or naming is used to replace truly understanding, the agency of those who have those experiences and understandings is undermined. This risk, that not naming an experience leads to it being ignored or undermined, is particularly problematic for cultures where there are certain things that can't be named or understood because they are outside the dominant hermeneutical framework entirely. When we miss such understandings, it is usually because knowledge is only counted as such if it fits the frameworks that are used by those in positions of authority, which usually draw on the dominant hermeneutical resource alone.

For example, Jill Stauffer discusses a case where oral history was used as the definitive evidence in determining which of two aboriginal groups, the Navajo or the Ute/Paiute, had the stronger claim on three ceremonial shields found in Utah. The Navajo claim was presented as a narrative about the shields' role in the history of the Navajo Nation (Stauffer 2015, 99). This oral history, which in decades past would already have encountered reduced credibility and understanding, was accepted because of attempts by the Native American Graves Protection and Repatriation Act (NAGPRA) to use wider standards of proof to grant "Native Americans' oral testimony the credibility it deserves" (Stauffer 2015, 97). The Ute/Paiute claim, however, was presented as "a more straightforward legal argument" (Stauffer 2015, 99), citing where the shields were discovered and their material and aesthetic similarities to known Ute traditions. The 
archeologist judging the case ruled in favor of the Navajo, citing the lower credibility of the Ute/Paiute claim as the reason for her decision (Stauffer 2015, 100). In this case, it seems that NAGPRA assumed the two different cultures could use oral history and oral traditions in the same way, and that the Ute/Paiute didn't use such a story because they didn't have one. However, in making that assumption NAGPRA failed to account for the cultural differences between the two groups that impacted how they could present their claim. The Navajo were able to use the oral tradition in the expected way, giving a story of how the shield fit into their beliefs and history. The Ute/Paiute, on the other hand, were unable to give their version of that kind of story because their beliefs include being unable to share information about their religion with outsiders (Stauffer 2015, 100). Because NAGPRA had included oral history as accepted (and in some ways expected) testimony for the specific purpose of closing a gap in communication and understanding between aboriginal groups and non-aboriginal legal bodies, it was unable to recognize when that tool was inadequate. This led to both testimonial and hermeneutical injustices against the Ute/Paiute claimants, even though the acceptance of oral histories was meant to avoid epistemic injustices that had been committed in the past.

\subsection{What Fricker is Missing}

Many of the critiques that are brought against Fricker's approach point to aspects of how epistemic injustice manifests in ways that her account misses. These critiques all draw out how the strict delineation of separate categories with fixed definitions within epistemic injustice creates a problem for Fricker's approach. That categorization leads to some cases of epistemic injustice falling through the gaps between the definitions of testimonial and hermeneutical injustice, while at the same time obscuring the complex 
relationships between those defined types. The problem posed by categorization itself will be discussed in section 2.3. This section will tease out the relevant implications of the critiques made by Hookway and Medina, Maitra, and myself.

\subsubsection{Epistemic Activities that are not Testimony}

In his critique of Fricker, Hookway specifically argues that there are "other kinds of epistemic injustice" $(2010,152)$, which do not seem to be covered by testimonial injustice and hermeneutical injustice because they occur between individual agents but do not happen "in the context of a straightforward testimonial exchange" (153). He objects that in labelling those epistemic injustices that occur in individual exchanges "testimonial", this category appears to only consider what is happening when one person tells another person something and provides straight forward testimony. However, the idea of epistemic injustice seems to be concerned with injustices that involve anything that constitutes "being engaged in epistemic activities" (154). Engaging in epistemic activities involves much more than just the exchange of information through testimony. The activities that someone engages in as a knower include such things as asking questions (155), and listening to someone's testimony with the purpose of contributing to the discussion, among others. All of these epistemic activities can also have their credibility undermined due to an identity prejudice on the part of other participants in the epistemic exchange. For example, there can be a form of epistemic injustice that does not discredit the truth or validity as knowledge of a person's testimony, but that instead discredits the agent's ability to provide information or participate epistemically in ways that are relevant to the situation at hand (158). Fricker's account seems unable to capture how these sorts of cases could be ones of epistemic injustice, since it does not fit the 
description of a hermeneutical injustice, but is also not a case of the speaker giving testimony that is then discredited. However, it does fit the description of epistemic injustice, because it undermines the individual in their capacity as a knower. This is why it is important to pay attention to the contextual and relational aspects of epistemic injustice, because there are many different ways that those contexts and relationships can lead to such injustices.

Medina also identifies epistemic activities that seem to fit most aspects of the definition of testimonial injustice, but which do not involve direct testimony. He argues that a speaker can be undermined in her capacities as a knower not only when she tries to share her knowledge through testimony, but also in her role as an inquirer trying to gain further knowledge. Medina argues that an epistemic injustice can occur, even when someone is treated as a full informant, if they are excluded from engaging in the authoritative communicative activities that are involved in producing knowledge through inquiry (2012a, 203-204). If discrediting people's capacities as inquirers is included in testimonial injustice, it has implications for hermeneutical injustice as well as testimonial injustice, as it blurs the lines between the two categories. The exclusion of people with certain social identities from inquiry and the productions of knowledge is what lies at the heart of hermeneutical marginalization and causes hermeneutical injustice.

Fricker's account can accommodate these objections, but they are still important to point out. In her response to Hookway's critique, she refers to a passage in Epistemic Injustice, where she explicitly states that testimonial injustice is not restricted to testimonial exchanges (Fricker 2010,174). The reason she calls her first category of epistemic injustice "testimonial" is because she thinks giving testimony, or telling, is the 
"parent case" of the type of injustice in which the basic wrong comes from undermining the speaker as a knower (174). She thinks that the ability to share knowledge through telling is central to what it means to be a knower. Since testimonial knowledge is at the foundation of human knowledge (176), she argues that it is important to highlight this aspect of the first category of epistemic injustice. However, she also allows that once attention has been successfully drawn to two fundamental forms of epistemic injustice, the categories may lose their value (176). In section 2.3, I will argue that the categories not only lose their value, but in fact cause additional problems for an effective account of epistemic injustice. The critique Hookway and Medina put forward help to demonstrate these problems.

\subsubsection{Problems with the Definition of Testimonial Injustice}

Maitra's critique focuses not on the type of epistemic activities that Fricker fails to discuss, but instead on how the definition of the category of testimonial injustice may catch some cases that do not actually count as an epistemic injustice while excluding some that do. She identifies three different aspects of Fricker's definition that may cause problems for accurately identifying testimonial injustices.

The first is Fricker's lack of attention to whether or not we actually owe it to certain speakers to judge their credibility correctly. While Maitra leaves unanswered questions regarding when and why we do not owe it to someone to judge their credibility correctly, she argues that there are some people to whom we do not have such an obligation $(2010,195)$. Despite the questions left unanswered in her argument, she raises an important point about the relationships between individuals and how the power dynamics between them will impact whether or not we attribute epistemic injustice to a 
particular situation. Those relationships and the power embedded in them are not adequately explored in Fricker's approach, and this lack of attention to relationships is a weak point in Fricker's theory.

The second aspect of Fricker's approach that Maitra critiques is the differentiation between epistemic injustice and epistemic bad luck. As mentioned in Chapter 1, Fricker's appeal to epistemic bad luck in the case of Greenleaf exempts him from blameworthiness for his epistemically unjust treatment of Sherwood. Maitra points to how the possibility of an appeal to epistemic bad luck may lead to some actual cases of epistemic injustice being dismissed (203). Maitra argues that many such cases should still be considered epistemic injustice, because the speaker's “capacity to impart knowledge, even about his own skills, is undermined, and as such, he is symbolically degraded qua human being. $\mathrm{He}$ is not treated as a subject of knowledge" (204). By explicitly excluding such cases from the category of testimonial injustice, Fricker reinforces some of the harm done to subjects such as Sherwood by denying that their experience is one of epistemic injustice. It also lets the powerful off the hook for failing to recognize that they have done something wrong when they follow these prejudices. Dotson's argument from section 2.1.2, that Fricker's framing of epistemic injustice with epistemic bad luck as its antithesis creates a closed conceptual structure that perpetuates epistemic oppression (Dotson 2012, 25), reinforces Maitra's concern.

Maitra's third critique argues against Fricker's emphasis on resistance to counterevidence as an important aspect of what makes a stereotype identity-prejudicial and therefore a source of testimonial injustice. Maitra argues that it is possible for a stereotype to not be resistant to counter-evidence, but to still be prejudicial, if the 
prejudiced person fails to seek out counter-evidence that would disrupt their identity prejudicial stereotype $(2010,206)$. Even if someone's prejudice is not resistant to counter-evidence, if that prejudice negatively impacts the credibility they attribute to someone because of that person's social identity, then the harms and wrongs of testimonial injustice will still arise. Someone could intentionally avoid being put in a situation that challenges their identity prejudice, preserving the prejudice by avoiding counter-evidence and in the process continuing the harms and the wrongs of testimonial injustice. This is yet another way in which Fricker's careful delineation of testimonial injustice excludes cases of epistemic injustice. Maitra's critique once again points to the importance of the relationships between dominant and marginalized groups and the role they play in the harms that prejudice causes. The relationships that someone chooses to cultivate or ignore will influence what counter-evidence they are exposed to, and will shape their prejudices and world view.

All three of Maitra's objections to Fricker's approach draw attention in an important way to the role that relationships play in epistemic injustice, a role that does not seem to fit with Fricker's approach. This is not only the role of relationships between the people involved in specific instances $(200,201 \& 203)$ but also the relationships between the two categories of testimonial injustice and hermeneutical injustice (195196). The strict definitions and categories of Fricker's account leave out important instances of epistemic injustice, and important factors that play a role in how the harms of epistemic injustice will manifest. 


\subsubsection{Discrediting Privileged Advocates}

Fricker's theory of epistemic injustice also fails to account for instances where privileged individuals may be discredited because they try to act as a voice for the marginalized, attempting to bridge the gap between the oppressed and the powerful. In such cases where the advocacy of a privileged person with regard to marginalized people is discredited, it is the content of the testimony and not the identity of the speaker that leads to the discrediting of that testimony. I would argue that this fits the broad concept of epistemic injustice, and contains both a severe wrong and a severe harm, but because it is experienced by someone in a position of privilege it does not fit neatly into Fricker's definitions of either testimonial injustice or hermeneutical injustice.

Take, for example, the role that Atticus Finch plays in Tom Robinson's trial as told in Harper Lee's To Kill a Mockingbird. Finch is a white man and a lawyer with a respected position in his community. He is generally in a position of epistemic privilege. However, he has done something epistemically exceptional by giving appropriate credibility to Robinson's testimony that he is innocent. When he in turn advocates for the innocence of his client (Fricker 2007, 25), it is not only his client's testimony but also his own testimony on behalf of his client that is disbelieved. Because this is not an identityprejudicial credibility deficit towards Finch, this is not a testimonial injustice according to Fricker's account. It is also not a hermeneutical injustice against Finch, because he is not part of a social group that has been excluded from meaning generation and it is not predominantly his experience that the jury is failing to grasp. It would seem to follow that the jury's discrediting of Finch's testimony is not an instance of either type of epistemic 
injustice that Fricker describes. However, an injustice has occurred, and it has a distinctly epistemic character.

The testimony that Finch is presenting is being given reduced credibility not because of the identity of the speaker, but because of what the speaker says. He is not believed specifically because of the content of the knowledge he is trying to give the jury, that of a black man telling the truth about not raping a white woman. The identity prejudice that the jury feels against Robinson causes them to give reduced credibility to that testimony even when Finch is the speaker. Finch is acting as an advocate, presenting the experience of someone who is being harmed by an identity-prejudicial credibility deficit, but he does not carry the visual indicator of being black. If the prejudice was only based on the identity of the speaker, then once the visual indicator of that identity was not present one would expect the credibility deficit to no longer be present either. Counterintuitively to Fricker's account, Finch's testimony also receives reduced credibility, resulting in an epistemic harm being done to Finch. However, the ethical harm is being done to Robinson. Robinson's experience and knowledge is being denied, even though it is not Robinson who is presenting it. In other words, an epistemic injustice seems to be done to both Finch and Robinson, since the credibility of both is being undermined.

This case demonstrates the important role that epistemic injustice has in reinforcing the power relations in society through oppression and marginalization. The discrediting of Finch's testimony, marginalizing a voice that advocates on behalf of Robinson and tries to reduce the harmful influence of racist oppression, ensures that the dominant societal understanding of what relationships are possible between white women 
and black men remains intact. This is why it is so important to situate instances of epistemic injustice in their social and political context, which includes the society's social understandings and the relationships between members of a community. In the context of the time in which the story is set, the idea that a white woman could desire a black man and a black man could feel sorry for a white woman was inconceivable to a white audience. That inconceivability helped to reinforce the domination of people of color by the white population, by dismissing and discrediting alternative possibilities that could shake the story of superiority on which dominance is built. This was the case even when those alternatives were presented by a white man. The power at stake in the accepted understanding results in the white jury turning on the white lawyer. As a result, the influence of prejudice against certain groups extends not only to the credibility of what is said by members of that group, but also of what is said about members of that group, by those challenging structures of oppression.

\subsection{Better Approaches}

While the previous sections focused on details that Fricker's account misses, this section will examine the broader approaches that could more accurately and effectively theorize epistemic injustice. Anderson calls for a more structural approach to epistemic injustice, with less focus on individual transactions. Hoagland, Medina, and Pohlhaus all push for increased relationality, particularly increased attention to the relationships between the different actors and factors at play in situations of epistemic injustice. Making a theory of epistemic injustice more sensitive both to structural aspects of epistemic injustice and to relationships that impact such injustices will help to improve its accuracy and its effectiveness. 


\subsubsection{Taking a Structural Approach}

Anderson draws attention to how the structural aspects of epistemic injustice are often lost in Fricker's account. She claims that in Fricker's account, both testimonial injustice and hermeneutical injustice are approached through a transactional theory of injustice (Anderson 2012, 165 \& 166). This transactional approach portrays hermeneutical injustice and testimonial injustice as the kind of injustice that is particular to "exchanges or interactions between one person and another" (163-164). However, Anderson thinks that epistemic injustice instead requires a structural account, one that "supplies criteria for assessing global properties of a system of rules that govern transactions, and imposes constraints on permissible rules with an eye toward controlling the cumulative effect of individual transactions that may be innocent from a local point of view" (164).

A structural approach, as opposed to the transactional account, brings attention to the aspects of epistemic injustice that require structural remedies rather than just individual ones (168). It will also bring attention to the fact that "structural forms of testimonial injustice are more pervasive than acknowledged in Fricker's work" (169). This fits with my argument, discussed earlier, that hermeneutical injustice and testimonial injustice can't be separated. Hermeneutical injustice, which is always structural (166), is always present and cannot be extracted from the testimony and transactions of individuals. It is the structural relations of domination, marginalization, segregation, and oppression, which turn ethically "innocent, if cognitively biased, epistemic transactions into vectors of epistemic injustice" (171). In many cases, only a structural approach can show the reason why what occurs in an epistemic transaction is unjust. An approach that follows Anderson in taking a structural approach to epistemic injustice would be able to 
place the individual transactions into the wider context of systematic injustices and bring attention to the relationships between those transactions and the structures that form the backdrop to these transactions and relationships.

Fricker's theory, through its transactional approach, focuses almost exclusively on "the speaker who is the target of a credibility assessment" (Medina 2011, 18) and on the subject who is prevented from understanding something that it is in their interest to understand. This focus is very individualist, failing to recognize the intricate social relations and societal structures that are at play in each injustice. Particularly in her description of testimonial injustice, she talks about the speaker and the hearer (Fricker 2007, 17) or $a$ speaker and $a$ hearer (1). This indicates a singular, individual person on each side of the injustice. However, as Medina points out in his criticism of hermeneutical injustice, it is not just one speaker and one hearer who are impacted by epistemic injustices, but everyone else who is connected to the interaction by their relationships to those participants (Medina 2011, 18).

This means that the harms our analysis of epistemic injustice needs to take into account aren't only the harms accrued by the marginalized person experiencing epistemic injustice, but also the harms the wrong of epistemic injustice causes to every other member of their social group. It also needs to account for the credibility excesses attributed to the dominant group, and how those credibility excesses result in corresponding credibility deficits to the marginalized groups with whom they interact. Epistemic injustice is not just about the individuals. The relationships between the dominant and marginalized members of society, as they are shaped by contexts and the networks of relationships in which they are embedded, are crucial to understanding 
epistemic injustice. These relationships can either produce or repress alternative ways of viewing the world, and can prevent or perpetuate epistemic injustices. The structures of the society, the institutions that help to shape those structures, and the communities that shape the institutions are all crucial aspects of those relationships, which are necessary for understanding how epistemic injustice is embedded in the very structures of society.

\subsubsection{Paying More Attention to Relationships}

The movement towards a more structural approach to epistemic injustice should be accompanied by a move to a more relational approach as well. According to Hoagland, relationality involves our "forming and being formed, both individually and culturally, in relation through our engagements and practices" (Hoagland 2007, 99). She argues that it is a lack of recognition of relationality and relationships that leads to epistemic injustice. Cases of what she calls motivated ignorance, such as white ignorance (which will be discussed in section 3.1.), involve the perpetrator failing to realize how their own beliefs, knowledge, and identity are formed by their social relations. They fail to recognise how that influence causes them to have a skewed view of the world. In cases of testimonial injustice, where someone gives someone else's testimony less credibility than it is due because of identity prejudice, the hearer is failing to recognize how prejudice has negatively impacted that credibility judgement as well as other relationships with members of the group they are prejudiced towards. If they correctly recognized the distorting impact of prejudice on how they relate to the speaker, then they should be motivated to readjust the credibility they attribute to the speaker. In cases of hermeneutical injustice, where a gap in the dominant or alternative hermeneutical resources leads to certain people not understanding certain experiences, part of what they 
fail to understand is their relationship to that experience and to the person experiencing it. They also fail to recognize how those outside the dominant hermeneutical resources are shaped by those relationships in ways that give them more or less credibility. The breakdown of relationships between individuals lies at the heart of most epistemic injustices, and so taking a relational approach will help the identification of such injustices.

Medina also urges theorists to take an approach that pays more attention to relationships. Such a relational approach will help to address many of his critiques of Fricker's approach, as has been mentioned previously. The effects of a credibility judgment on different subjects involved in an epistemic activity are not independent and cannot be separated from each other (Medina 2011, 18), because everyone is caught up in formative relationships with others. Paying attention to the relationship between credibility excess and credibility deficit, where the excess credibility attributed to someone in a position of privilege will almost always correspond to a credibility deficit to members of a different social group, will reveal important information about the power structures of society and how they impact how knowledge is judged. Looking at the relationship between power structures in society and where hermeneutical marginalization leads to hermeneutical gaps around the experiences of particular social groups will also help us to understand how everyone who is complicit in those structures of domination will in effect be a perpetrator of hermeneutical injustice. The relational nature of the injustice makes everyone responsible for doing what they can to prevent it, and those who fail to prevent it will either perpetrate or perpetuate it. It will also help to show what is gained by way of knowledge of those structures from the situated 
perspective of those who are marginalized, knowledge that is often missed by the dominant.

Furthermore, credibility judgments are deeply embedded in their hermeneutical context and the epistemic resources available to the participants. This is why the existence of alternative hermeneutical resources is so important to accurately theorizing epistemic injustice. Their existence undermines Fricker's use of epistemic bad lack as an exemption from blameworthiness. The relationships between the members of a marginalized group, and between different marginalized groups, which allow alternative hermeneutical resources to develop among those who are excluded from the dominant hermeneutical resource will also be important for understanding how it is possible for resistant systems of understanding to arise. The relationship between these alternative hermeneutical resources and the dominant groups and dominant hermeneutical resources will also be important, because it is through those relationships that the wrongs and harms of epistemic injustice will still arise even when alternative hermeneutical resources are available. All of these relational aspects need to be recognized in the analysis of epistemic injustice.

\subsection{The Problem with Categories}

It is not only the relationships between the people and aspects of their society that need to be examined. Through this analysis of the critiques of Epistemic Injustice, it has become evident that there is a deep, complex, and essential relationship between hermeneutical and testimonial injustice, to the point where separating epistemic injustice into these two categories is an inaccurate and inadequate approach. Even with her insistence that the categories of testimonial injustice and hermeneutical injustice are not exhaustive and that 
there are other forms of epistemic injustice, the use of categories gives the impression of delineating what is meant by epistemic injustice in a way that is exclusionary. There are many examples of types of injustice that undermine the individual in their capacities as an epistemic agent but do not fit into the two categories of epistemic injustice that Fricker defines, nor in their sub-categories. In defining those two categories and not discussing epistemic injustice outside of those categories, there is an implication that what falls outside of those two definitions is not of concern to those interested in epistemic injustice.

As is pointed out by Hookway, Medina, and Maitra, what falls outside of Fricker's definitions of those two categories often includes very important epistemic activities. It has implications for how prejudice impacts those epistemic activities in ways that harm marginalized individuals. The inadequacy of categorization would likely be true even if Fricker explicitly defined and discussed more categories beyond testimonial and hermeneutical injustice. There will almost always be cases with ambiguous characteristics, which don't fit neatly into the categories that have been defined but that contain the characteristics of the general concept of epistemic injustice. This is the feature of epistemic injustice that only becomes clear through an examination of the complex structures, contexts, and relationships involved.

The categorization of testimonial injustice as separate from hermeneutical injustice also artificially separates the participants from their relationships with other people and with their context, the opposite of what we want in a more structural and relational approach. Although Fricker describes testimonial injustice as taking place between individuals, in the realities of testimonial exchange there is more than just the 
speaker and hearer involved. The interlocutors will have relationships with witnesses to the exchange, with other members of one's social group, with the members of other social groups, with political structures, with the dominant hermeneutical resource, and other relationships as well. All of these relationships and people need to be considered. Since testimonial injustice by itself does not account for these relationships, Fricker's approach to testimonial injustice as distinct from hermeneutical injustice cannot always fully elucidate the epistemically unjust harms that arise in complex real-world contexts. My expanded discussion of Fricker's examples throughout this chapter shows that in both the case of Sherwood and the case of Robinson, the individual and the epistemic injustice done to them cannot be extracted from the broader structures and relationships in which they are embedded. The same can be said about other cases of epistemic injustice.

Fricker's discussion of credibility excess and incidental cases of hermeneutical and testimonial injustice is one example of the account's short comings in this regard, as mentioned previously. Fricker denies that credibility excess can be a testimonial injustice, except perhaps in cases where the effect is cumulative $(2007,21)$. Medina, however, argues that she is taking too narrow a view of the impact of credibility excess. If credibility excess attributed to those in positions of privilege is examined using a broader view that sees credibility as interactive, extended over time, and impacting people beyond just the two individual interlocutors, it becomes clear that credibility excess does contribute to testimonial injustice. Medina points out that when one person is judged with a credibility excess based on their social identity, then there will be a corresponding decrease in the credibility of those who do not share that social identity 
$(2011,22)$. This is because when credibility is judged based on identity prejudice it will be a comparative or contrastive judgement.

For instance, if a man is given more credibility specifically because he is a man, then this will almost always result in a woman in the same position being given less credibility specifically because she is a woman, because their credibility will be judged in relation to one another (19). If someone is given more credibility specifically because of their social identity, such as for being a man, then someone with the opposite social identity, such as a woman, will be given less credibility because of their identity. As a result, the credibility excesses given to those in positions of privilege cannot be ignored by those want to understand the harms and wrongs of epistemic injustice (20-21). The definition of testimonial injustice as occurring in individual exchanges obscures the complex relationships between credibility excess and credibility deficit that Medina points out.

Fricker's categorization also artificially separates identity prejudicial credibility deficits in individual exchanges from gaps in understanding stemming from the epistemic marginalization of certain social groups. These two aspects of epistemic injustice cannot be so easily pulled apart into different categories of epistemic injustice. In his critiques of Fricker, Medina emphasizes the interconnection of testimonial and hermeneutical injustice, arguing that the two phenomena are often so closely interrelated that we cannot understand one without the other (2012a, 206). Maitra also discussed how closely connected the two categories are, arguing that testimonial and hermeneutical injustice each contain both individual identity prejudice and structural identity prejudice (2011, 209). Fricker's account of the two types of epistemic injustice tends to designate 
individual identity prejudice as causing testimonial injustice, while structural identity prejudice is found in cases of hermeneutical injustice. However, Maitra rightly points out that individuals in testimonial exchanges draw on structural resources to determine the credibility of a speaker. At the same time, hermeneutical injustices are reinforced by individual identity prejudices that undermine attempts to develop the conceptual resources to understand certain experiences (209), making the two types of epistemic injustice inseparable.

For example, Maitra builds on Fricker's examples from To Kill a Mockingbird and The Talented Mr. Ripley, and argues that "Tom Robinson and Marge Sherwood both suffer as they do because certain racist and sexist stereotypes are so easily accessible and so powerful in the collective understanding" (209). These stereotypes are structural identity prejudices, often stemming from the hermeneutical marginalization of certain groups. However, in the examples as they are given by Fricker the injustices manifest as individual identity prejudices revealed in testimonial exchanges. This individualist focus allows Fricker to attribute responsibility or a lack of responsibility to individuals in these exchanges without considering their broader context. Considering this, Maitra proposes that it may be the case that categorizing epistemic injustices into testimonial and hermeneutical injustice should not be done at all, since any incidence of epistemic injustice will include varying degrees of each type (210). I concur, and will develop an account of epistemic injustice without division into categories in Chapter 3.

\subsection{In Summary}

So far in this project, I presented Fricker's theory of epistemic injustice as it is articulated in Epistemic Injustice. I then provided a selection of the critiques that have been leveled 
against Fricker's account that I think most convincingly identify the greatest flaws in her approach. I have also indicated the direction I think an improved theory of epistemic injustice should go, moving away from categorization and towards a more structural and relationship focused approach. In the next chapter, I will argue for a new approach to epistemic injustice that addresses the concerns raised in this chapter. This new approach will be used in the final two chapters to discuss how epistemic injustice impacts global development in the real world, an application that is missing in the literature on epistemic injustice thus far. 


\section{Chapter 3: The New Approach}

In this chapter, I will examine white ignorance as an example that cannot be adequately theorized under Miranda Fricker's account. I take understanding white ignorance to be a crucial element in understanding the complexities of how epistemic injustice manifests in the real world. I will continue to use the term "epistemic injustice", as I intend to develop an alternative approach that still uses the same general concept of epistemic injustice as does Fricker's, but takes a different approach to its analysis and conceptualization. After discussing the phenomenon of white ignorance, I will draw on the work of José Medina, Maria Lugones, and Lorraine Code to build a new approach to epistemic injustice. This new approach will develop what emerges from the critiques raised in Chapter 2, and will also provide a more effective way to examine the epistemic injustices that arise in global development, which I will discuss in Chapter 4. To achieve this, my new approach will be more structural, contextual, relational, and attentive to alternative hermeneutical resources.

\subsection{White Ignorance}

Charles Mills defines white ignorance as a specific epistemology of ignorance, where "a particular pattern of localized and global cognitive dysfunctions...produc[e] the ironic outcome that whites will in general be unable to understand the world they themselves have made" (Mills 1997, 18). For Mills, this is particularly evident in unequal power relations between races and the oppression of people who are not white. This is because whites are unable "to recognize [their] own racial identity and the presuppositions and consequences of [their] racial positionality" (Medina 2012a, 212). Alison Bailey describes it as "a form of not knowing (seeing wrongly), resulting from the habit of 
erasing, dismissing, distorting, and forgetting about the lives, cultures, and histories whites have colonized" (Sullivan and Tuana 2007, 85). Sharon Sullivan and Nancy Tuana argue that it includes "both false belief and the absence of true belief about people of color, supporting a delusion of white racial superiority that can afflict white and nonwhite people alike" (3). In other words, white ignorance is what happens when people believe the story of white superiority that has been constructed over centuries, grounded in the idea that being "white" is the default and does not carry with it a racial identity at all. White ignorance is a distorted view of the world that misunderstands race in such a way as to re-entrench structures of domination, subordination, oppression, and deprivation.

White ignorance is experienced not only by white people themselves, but by people of all races who have been taught the same narratives and draw on the same hermeneutical resources that result in the same cognitive dysfunctions. The concept is multidimensional, and can be experienced by members of different social groups in different ways depending on their context and their perspective. Someone can be black and still internalize the concept of white racial superiority based on the inability to recognize the racial identity and the positionality of white people and its consequences (Mills 2013, 38-39). The harms of white ignorance are also experienced by both people of color and the white population that it privileges. For people of color, it produces ethical and practical harms such as marginalization and oppression resulting from the assumption of white racial superiority. It shapes societal structures and social, political, and economic interactions in ways that negatively impact people of color, and it produces epistemic harms as it prevents people of color from understanding the reality of the world 
that they live in, or their role in it. For white people, white ignorance prevents them from understanding the role of race with respect to their own identity and their position in the world. The most obvious harm to white people is epistemic, because it robs them of the ability to see the truth about the world they live in. However, they also will be indirectly impacted by the practical harms that white ignorance causes people of color, because the white population loses the beneficial contributions that people of color can make to shared epistemic activities and the practical benefits that result from those contributions.

Mills also draws attention to the ways in which white ignorance provides a barrier to non-whites trying to insert alternative hermeneutical resources into the dominant hermeneutical resources. It prevents "white comprehension of the true nature of the social order, or some crucial local section of it", and therefore perpetuates hermeneutical injustices (Mills 2013, 42). When those who control the dominant hermeneutical resource are able to justify or obfuscate the domination that is involved in the most prominent and dominant world view and hermeneutical resource, it becomes even more difficult for alternative views to make headway in introducing new ideas to fill the gaps that cause hermeneutical injustice. Even though this "paucity of concepts" (Fricker forthcoming, 16) may only exist for white people, the ethical harms it creates cause a greater disadvantage to people who are not white, and as a result it is very much in the interest of the marginalized individuals who are most harmed by white ignorance to have their experience understood by those who are experiencing the cognitive dysfunction of white ignorance.

Several of Fricker's critics, including Rebecca Mason (2011, 303), Medina (2012a, 214) and Gaile Pohlhaus Jr. $(2012,722)$ have suggested that white ignorance is 
an example of hermeneutical injustice. However, if it is a hermeneutical injustice, it presents an anomaly for Fricker's view (Medina 2012a, 21). This is because in white ignorance "the hermeneutically disadvantaged are not those who suffer the practical consequences" (214). If, as Fricker claims, hermeneutical injustice consists of being prevented from understanding experiences that are "in your interest to render intelligible" (214), then when white ignorance prevents a white man from understanding experiences that would make him question his position of privilege and his understanding of the world, it could not be a hermeneutical injustice to him on Fricker's account because it is not in his interest to question his privilege. Take, for example, a white man who denied the existence of systemic police violence against people of color. Although that white man is at an epistemic disadvantage, because he does not recognize this systemic injustice is taking place, he gets practical benefits from the lack of understanding on his part and that of those like him. It would therefore not be in his interest to understand the knowledge he is missing. According to Fricker's account, no hermeneutical injustice has occurred.

However, Medina's critique of Fricker's account of hermeneutical injustice (214215) reveals how the white man's white ignorance about police violence would cause a hermeneutical injustice to people of color. In this example, someone with power and privilege in the society is failing to understand the experiences that many people of color have with police violence. It is in the interest of those who encounter police violence because of their race to have that experience understood by those who could help to influence changes in the society's oppressive structures and thereby prevent that structural injustice. White ignorance prevents those in positions of privilege, specifically 
white people, from understanding the experiences of other races and from understanding why changes in the structures of society are necessary. This causes practical harms to marginalized people, even if they don't suffer a direct epistemic disadvantage. In the example of the white man experiencing white ignorance about police violence towards people of color, both an epistemic and an ethical harm occurs, just as is required for an epistemic injustice under Fricker's definition. However, the epistemic harm is experienced by the white man, who fails to understand the experience of many people of color, while the ethical harm falls on the people of color whose experiences of racially motivated police violence are not heard or understood by the white man in a position of structural power (214). The hermeneutical injustice is done to the people of color who continue to suffer from a structural injustice because white ignorance continues to marginalize their experiences from the understanding of the dominant hermeneutical resources and those who have the most power to change the unjust structures that emerge from them. The relationship between the white man's white ignorance and the continuing violence done to people of color is an essential part of understanding the structural injustice taking place and the role of epistemic injustice in perpetrating that structural injustice.

Alternative hermeneutical resources play a powerful role in countering the harms of white ignorance, a role they play in any case of hermeneutical injustice and epistemic injustice more generally. The Black Lives Matter movement is one such example, where those who have experienced racialized police violence and those who stand in solidarity with them are using their knowledge of their experience to try to counter the dominant hermeneutical resources that discredits, denies, and fails to understand those experiences. 
It is a powerful example of the potential of alternative hermeneutical resources to bring the plights of marginalized groups the attention of dominant groups in society. However, it also shines a light on how white ignorance resists counter-evidence that could disrupt the power structures and assumptions of the dominant hermeneutical resource. When those who oppose Black Lives Matter introduce the counter slogan "All Lives Matter", they are trying to draw attention away from the specific experiences of people of color that Black Lives Matter protesters are trying to introduce into the dominant hermeneutical resource. They are obscuring the structural and racialized injustice that is occurring.

Through this discussion, the important role that white ignorance plays in epistemic injustice should now be evident. Fricker does discuss white ignorance in her more recent work on epistemic injustice. However she does not think that it belongs in the category of hermeneutical injustice. She instead proposes it be included in a third category of epistemic injustice, not mentioned in Epistemic Injustice, which she calls "motivated ignorance" (Fricker forthcoming, 2). The addition of this third category allows her to include white ignorance as a kind of epistemic injustice, without having to change the definitions of testimonial injustice or hermeneutical injustice to include it. Fricker designates white ignorance to this separate category because in such cases "it is not whites who are broadly disadvantaged by the hermeneutical lacuna- they are epistemically disadvantaged, but their lack of conceptual tools with which to know their own social world plays to their general social advantage" (Fricker 2013, 52). According to Fricker, for white ignorance "to constitute a hermeneutical injustice the deficit of concepts in the white population would have to be unfair to them in some way" (Fricker forthcoming, 21), and this cannot be the case when the "epistemic disadvantage is more 
generally to [white peoples'] social advantage" (Fricker forthcoming, 21). It is unclear what Fricker thinks would be sufficiently unfair to white people in order to count as a hermeneutical injustice.

Although Fricker's new category of motivated ignorance succeeds in incorporating white ignorance into her account, it is still inadequate to fully encapsulate the role that white ignorance plays in epistemic injustice. She creates a completely separate category of epistemic injustice characterized by "a certain kind of collective interested or motivated cognitive bias in what social interpretations and/or evidence for such interpretations a racially dominant group attends to and integrates into the rest of their beliefs and deliberations" (Fricker forthcoming, 16). This definition would usually lead to the exclusion of alternative hermeneutical resources, and through this exclusion Fricker obscures important characteristics of the phenomenon of white ignorance. Her characterization of motivated ignorance in the above quote focuses only on the racially dominant group and the epistemic disadvantage caused by the "motivated cognitive bias" at play. This focus does not account for the epistemic and practical harms that motivated cognitive bias causes for people of marginalized groups.

It also does not account for the ways in which members of marginalized groups can also experience white ignorance. Mills emphasizes that not only white people experience the cognitive bias that reinforces white supremacy (Mills 2013, 40). It would seem that in Fricker's account, a white person experiencing white ignorance would fall into the category of motivated ignorance, while a person of color experiencing white ignorance would be experiencing a hermeneutical injustice. However, this division of the different instances of white ignorance into different categories of epistemic injustice 
depending on the race of the person experiencing the cognitive bias fails to properly account for how the relationships between the two races and their structural inequality produces white ignorance and its harms. To be properly understood, white ignorance needs to be looked at more holistically. A white person's epistemic disadvantage caused by white ignorance, a person of color's epistemic disadvantage caused by white ignorance, and the practical harms caused to people of color by white ignorance are all different facets of the same phenomenon, a phenomenon that cannot be entirely understood if these facets are separated from each other. White ignorance cannot be properly understood using the categories of testimonial injustice, hermeneutical injustice, and motivated ignorance. It contains aspects of all three of Fricker's types, and cannot be understood through her divided lens.

White ignorance provides an excellent example of an injustice that is obviously epistemic in nature, but which Fricker's categories fail to adequately incorporate. It also provides an illustration of how the very process of categorization can obscure what is really happening in certain phenomena, a critique raised in the previous chapter under section 2.4. To understand white ignorance, one needs to understand the relationships at play between the ideologies of white dominance, the motivated cognitive biases of white people, the cognitive biases of people of color who have accepted the ideologies of white dominance, the suppression of alternative hermeneutical resources that could challenge those ideologies, and the practical harms that white dominance cause to people of color, among other factors. As a result, this discussion of white ignorance should persuasively illustrate why it is necessary to change our approach to epistemic injustice, in order to 
more accurately theorize this form of injustice and to more effectively address its impact on structures of domination and oppression in the real world.

\subsection{Moving Beyond the Logic of Purity}

To construct a new approach to epistemic injustice that resolves some of the flaws in Fricker's approach, I will first have to dismantle the very commitment to categories that she imposes. Lugones' discussion of the "logic of purity", and how to move away from it, can give insight into why it is important to remove the categorization of epistemic injustice while also providing a suggestion for an alternative approach. Lugones describes the logic of purity as "the attempt at control exercised by those who possess both power and the categorical eye and who attempt to split everything impure, breaking it down into pure elements... for the purposes of control" (Lugones 2003, 123). According to her, this is expressed in modern ethics "in an understanding of reason as reducing multiplicity to unity through abstraction and categorization" (127). Fricker's approach to epistemic injustice seems to fit this logic of purity, simplifying the complex realities of this phenomenon into abstract categories that allow each category to appear bounded and united under one definition. There is an "assumption of unity underlying multiplicity" (128), where the multiplicity is the wide variety of differences in detail that exist among different cases of each type of epistemic injustice and the assumption of unity is the assumption that they can be neatly divided into categories. Lugones claims that this construction of unity in modern ethics allows us to construct the "ideal observer" (129), the objective perspective of reason that Fricker and others like her seem to be using to analyze the injustice. This ideal observer tends to fit the dominant view of an objective, rational, impartial observer, which almost always defaults to a straight, white, cisgender, 
neuro-typical, able-bodied, middle income male. That in itself is problematic from the point of view of epistemic injustice, because it automatically discredits other social groups as not fitting the "ideal".

However, Lugones' main concern with the logic of purity and its construction of the ideal observer goes beyond the ways in which the construction of the ideal observer tends to reinforce structures of domination and marginalization. She also argues that "the ordering renders something out of place. [That thing's] complexity is altered by the ordering" (133). Something important about what she describes as the "multiplicity" of the thing, in this case epistemic injustice, is lost if we try to create neat, unified categories strictly separated one from the other. She describes how in the case of her own self "[she] realize[d] that separation into clean, tidy things and beings is not possible for [her] because it would be the death of [herself] as multiplicitous" (134). I think that the same problem occurs when the logic of purity is used to analyze epistemic injustice, where the multitude of variations among the manifestations of the concept and the complexity of its realization in different contexts is altered and obscured by the attempt to neatly categorize the phenomenon. Something important is lost in trying to tidily categorize epistemic injustices into different types with clear boundaries, because there is an insurmountable multitude of different ways in which it manifests that need to be recognized. Recognizing the multiplicity of selves in individual human beings will also play an important role in how some of the flaws of Fricker's approach to epistemic injustice can be resolved, and will be discussed in section 3.3.

In contrast with the logic of purity Lugones outlines the logic of curdling, which sees "the social world as complex and heterogeneous and each person as multiple, 
nonfragmented, embodied" (127). Lugones describes this logic using the allegory of making mayonnaise. The logic of curdling recognizes the kind of separation that occurs in the curdling of an oil and water emulsion, where the separation is not a complete split but instead where "they coalesce toward oil or toward water... a matter of degrees from coalescence" (122). Unlike the logic of purity, which delineates what is separated into different categories or fragments, the logic of curdling treats separation and difference as a matter of degree. This makes it better able to accommodate the complexity and multiplicity of the contexts and relationships that help to form human beings, recognizing that something is lost when the many aspects are split and separated. While Fricker's theory of epistemic injustice sought to split instances of epistemic injustice into testimonial injustice, hermeneutical injustice, or motivated ignorance, I propose a new approach that recognizes that each instance of epistemic injustice will necessarily consist of varying degrees and combinations of all Fricker's types1, as well as elements that are not explicitly discussed in her theory and which may or may not be unique to that particular context. The logic of curdling should also be applied to the people involved in epistemic injustice.

\subsection{Moving Towards a Complex View of the Self}

Lugones' conception of the multiplicity of the self is an important contribution to understanding the harms and wrongs of epistemic injustice. Lugones draws attention to Iris Marion Young's argument that "individual persons, as constituted partly by their group affinities and relations, cannot be unified, themselves are heterogeneous and not

1 I am not suggesting that Fricker thinks epistemic injustice in whatever form does not come in degrees, only that she does not go far enough in demonstrating the fluidity and complexity of these combinations. 
necessarily coherent" (Young 1990, 48). Jill Stauffer expresses a similar view. She argues that the self is "exposed, vulnerable, and formed in part in relation to others" (Stauffer 2015, 23). Both of these views of the self share the idea that it is not a unified, fixed, separate thing belonging to each individual in isolation but instead is variable, contextual, and deeply influenced by relationships. This is a crucial aspect of the literature defending a relational concept of the self. However, Lugones takes this a step further. She claims that Young is still using the logic of purity in viewing the subject as fragmented (Lugones 2003, 139). Lugones argues that the logic of curdling needs to be used to recognize the interlocking or intermeshing features of oppressions (Lugones 2003, 141). These features exist together in the person, shaping the self and making them neither whole nor fragmented but both at the same time. The result of using the logic of curdling to look at the self is to recognize this multiplicity of the self. The self is both many separate identities and one, with the person's identification with different aspects of that self varying by degrees depending on context, relationships, and other factors that shape them and shape the moment they are in.

This multiplicity of the self is important to recognize in an account of epistemic injustice, because different people will have different aspects of their multiplicitous identity rise to the surface in different contexts. Fricker's discussion of epistemic injustice seems to simplify these selves, making it easy to identify who is the "victim" and who is the "perpetrator" in a way that is not accurate to how epistemic injustice plays out in real life. In the example of To Kill a Mockingbird, it is clear that Tom Robinson is the victim, and the jury perpetrates the injustice. However, Fricker spends little time examining the impact of the situation on Mayella Ewell. As a poor, white, woman Ewell 
is both a victim and a perpetrator. She is a victim because she was beaten by her father, because her class and sex have led to her oppression and marginalization in society, and because in many circumstances she would be judged with a credibility deficit. It is likely that if she told the truth about what happened between her and Robinson, she would not have been believed either. However, she lies to the court, and in doing so she increases the credibility deficit that Robinson experiences, and is at least in part directly responsible for his conviction. It is also significant that Robinson is a black man, and Ewell is a white woman, but Fricker gives little attention to the gendered dimension of the situation.

Those involved in instances of epistemic injustice cannot be clearly split into victims, perpetrators, and innocent bystanders. In most cases, they will play different roles in different instances of epistemic injustice, and sometimes they will play multiple roles in the same instance. The roles people want to play, such as Ewell wanting to identify with and be accepted by the dominant white community, will also influence their identity in complex ways. An account of epistemic injustice should recognize that the social identity an individual embraces will shift depending on their context and the changing circumstances in their lives. This is true about social identities beyond sex and gender as well, including but not limited to class, ability, sexual orientation and nationality. As Lugones draws attention to the multiplicity of the self, she also draws attention to the ways in which relationships with different individuals and groups of people can bring different aspects of that multiplicity to the fore without ever eliminating the others. Fricker's account of epistemic injustice does not adequately emphasize the complexity of the different roles and relationships that can arise between the different 
selves involved in an epistemic encounter that results in epistemic injustice. Her examples are too clear cut. It seems to me that in Fricker's account Robinson is the victim, the jury is the perpetrator, and Ewell is a bystander (in the epistemic injustice at least). However, as discussed above, that does not tell the full story. Something similar happens in Fricker's other examples. Marge Sherwood is the victim, and Herbert Greenleaf is the perpetrator who is not blameworthy because he is experiencing epistemic bad luck. Carmita Wood is the victim who does not understand her own experience and cannot communicate it, and there is no perpetrator. In these examples, like the one from To Kill a Mockingbird, the situation is much more complicated and the roles of victim, perpetrator, and innocent bystander are much less clear cut. Paying attention to the specific relationships between the individuals and each person's relationship in the broader context will help to tease out the complexities of these multiplicitous selves in order to provide a more accurate picture of what is happening in epistemic injustice. Paying attention to this multiplicity will also give more substance to the agency of those harmed by epistemic injustice. Those who are often seen as "victims" are able to react to the specific demands of the relations of oppression they are in, enacting their own beliefs and values in reaction to their situation.

\subsection{Ecological Thinking}

Applying a logic of curdling rather than a logic of purity, and recognizing the multiplicity of the self, renders my theory of epistemic injustice more attuned to the complexities of how epistemic injustices manifest in the real world. Examining epistemic injustice through Code's approach of ecological thinking will attune my approach further to the relationships, context, and reality of epistemic injustice. Ecological thinking is "a 
revisioned mode of engagement with knowledge, subjectivity, politics, ethics, science, citizenship, and agency that pervades and reconfigures theory and practice" $(2006,5)$. It is "self-critically cognizant" of subjects' interdependent and situated relationships to both the social and the physical world (Code 2006, 5). It moves away from "the abstract, interchangeable, autonomous individual" (Code 2006, 5) that is central to the "orthodox epistemologies and ethical theories" in which oppression has been endemic (Code 2006, 6). Consequently, it is a more appropriate approach to epistemic injustice.

Lugones' account of the logic of purity is a part of what Code refers to as "a discourse of mastery", which she argues comes from and reinforces a social imaginary that "enlist[s] ready-made, easily applied categories to contain the personal, social, and physical-natural world within a neatly manageable array of 'kinds,' obliterating differences in a desire to assemble the confusion of the world into maximally homogenous units" (Code 2006, 19). Ecological thinking tries to move away from that discourse of mastery, introducing "a radically innovative conceptual apparatus" that undermines this hierarchical and divisive epistemology in favor of a theory of knowledge that is more sensitive to "a conception of materially constituted and situated subjectivity for which place, embodied locatedness, and discursive interdependence are conditions for the very possibility of knowledge and action" (Code 2006, 20). Code argues that this conceptual apparatus can resist "superimposing a ready-made cognitive grid upon events and situations, tucking in the bits that spill over, letting aberrations fall through the cracks" (Code 2006, 18) and thereby avoids the logic of purity.

It can be easily seen how ecological thinking both demands and helps to facilitate a more contextual, relational, and structural approach to epistemic injustice that moves 
away from the logic of purity. It directly targets the kind of categorization and individual focus that Lugones critiques. Code takes the kind of critically reflexive self-awareness that Fricker argues we should use to counter epistemic injustice (Fricker 2007, 91) and instead uses it to argue against the methods Fricker employs in building the rest of her argument. Ecological thinking allows us to understand epistemic injustice as a more holistic phenomenon, embedded in subjectivities of people who are constituted by their context, conditions, and relationships; the multiplicity and intersectionality of their identities; and their interdependence with other inherently social beings and the world in which they live. It also allows greater recognition of the complexities, contradictions, and instabilities of epistemic injustices and the people who experience them as they manifest in the world. Using the framework of ecological thinking to approach epistemic injustice facilitates a more structural, more contextual, more relational, and in the end a more emancipatory approach to understanding epistemic injustice than that offered by Fricker.

\subsection{A New Approach to Epistemic Injustice}

I will now present a more extensive account of the broader category of epistemic injustice that results from my new approach. As mentioned earlier, in Fricker's book very little is said about the overarching concept of epistemic injustice, despite it being the title. Fricker states that her intent in Epistemic Injustice is "to home in on two forms of epistemic injustice that are distinctively epistemic in kind, theorizing them as consisting, most fundamentally, in a wrong done to someone specifically in their capacity as a knower" (2007, 1, my emphasis). Although this is a good starting point, it does very little to elucidate how the problem of epistemic injustice should be understood outside of those two forms. Fricker's discussion of testimonial injustice and hermeneutical injustice can 
be used to infer some aspects of how she would conceptualize epistemic injustice as a whole, but that is insufficient for a theory of epistemic injustice that wants to do away with her categories entirely.

An epistemic injustice is a wrong done to someone in their capacity as a knower, which results in both epistemic and practical harms. The primary harm of this kind of injustice will always be the harm that comes from being wronged in a capacity essential to human value, since a person's capacities as a knower are a fundamental aspect of the human capacity for reason. This intrinsic harm will manifest in different ways, such as undermining the person's capacity to give knowledge, to have their knowledge understood by others, to gain knowledge, to contribute to the production of knowledge, and so on. Several of these different manifestations of the harms of epistemic injustice may arise in any particular situation, and each will occur in varying degrees. The ways in which these harms are related to each other and to the essential wrong done to the person in their capacity as the knower will vary.

The causes of this primary harm will usually be the negative influence of prejudice, marginalization, and oppression on how someone's epistemic capacities and activities are viewed due to their perceived social identity. Factors that will play an important role in causing epistemic injustice will include, but are not limited to, unfair credibility excesses and deficits, hermeneutical marginalization from dominant and/or alternative hermeneutical resources and their production, being prevented from giving knowledge, and not being heard by those to whom they are attempting to give knowledge. Which causal factors arise and how they present themselves will depend on the people involved, their history, where they are, their relationship to each other, their 
relationship to people in their social group, their relationship to people in different social groups, and other such context specific details. A variety of practical and additional epistemic harms can also result from epistemic injustice, and these too will depend on the context and the relationships of the people or groups impacted by the injustice.

In the background of the transactional exchanges and relationships between groups and members of groups, the structural inequalities and institutionalized power relations of the society in question will also influence how epistemic injustice manifests, what harms it will cause, and who it will harm. In analysing epistemic injustice, it will be necessary to understand both the societal structures and the personal relationships that are at play in any given case. It will also be necessary to keep in mind that each person is a member of multiple social groups at any given time, and different aspects of their multiplicitous self will come to the fore in varying degrees depending on the context they are faced with and what they may need to do to be heard or to challenge the status quo. This will impact the degree to which they experience marginalization and oppression, or privilege and dominance, in any particular case.

This approach to epistemic injustice will also require looking beyond what is happening in the dominant epistemic spaces, to the sources of resistance and innovation that can be found in marginalized communities. Situations of epistemic injustice will encounter the resistance of the people harmed by those injustices, using methods such as the creation of alternative hermeneutical resources, demanding structural change, and asserting their agency and epistemic capability despite the prejudices and marginalization imposed by dominant power structures. It is in these sources of resistance against 
epistemic injustice that attempts to mitigate and eliminate this form of injustice will begin.

Understanding epistemic injustice requires understanding that a person or a group can be victim and perpetrator, oppressed and resisting, innocent and implicated at the same time. Lugones' logic of curdling and multiplicity of the self demonstrate how this is the case. Code's ecological thinking then grounds this approach to epistemic injustice in its context, in the embodied relationships of people in the real world whose epistemic activities have practical effects within the unequal and often oppressive structures of society. Starting from an agent's situatedness in the context of those structures and relationships will help us to see how epistemic injustice unfolds. I turn to examples situated in particular contexts in Chapters 4 and 5.

\subsection{In Summary}

This chapter has argued that movement away from the categorization of epistemic injustices is the best way to address the harmful ways in which epistemic injustice has shaped both the everyday lives of individuals and the entire structure of our society over the course of history. Looking at epistemic injustice through a logic of curdling and with a model of ecological thinking builds a theory that pays attention to its structural basis, incorporates the importance of relationships, grounds analysis in embodied context, and opens up the possibilities for resistance that can lead to eradicating the wrongful harms it has caused. In the end, we have a new approach to epistemic injustice, one that can more adequately express the complexity of the issue.

The next chapter of this thesis will now apply my approach to epistemic injustice to the context of global development, and both theory and practice. Little has been done 
so far in the literature to apply the insights of epistemic injustice to these contexts, and that has shaped both how epistemic injustice is understood and how global development is understood. To begin to fill that lack, I will provide an overview of the many different ways in which epistemic injustices occur in different areas and at different levels in global development, examining several examples that emphasize important aspects of this new approach to epistemic injustice. The final chapter will provide even more detail, applying my theory of epistemic injustice to a case study of Cuba's health and educational systems, and the epistemic framework on which they are built. In this discussion, I hope to illustrate how epistemic injustice can occur at the level of epistemic frameworks, and how Cuba's particular history has led it to be the subject of epistemic injustice that discredits and marginalizes its contributions to global development efforts and its achievements at a domestic level. 


\section{Chapter 4: Global Development}

Although there is a large and growing body of literature that has emerged around Epistemic Injustice and its applications, there has been limited discussion about the epistemic injustices that occur in the context of global development. This chapter will contribute to that aspect of the literature by discussing several examples of the epistemic injustices that arise in global development policies and practices.

Arturo Escobar's work in post-development theory raises important questions about the meaning of development itself, which need to be considered when originating from the point of view of people in "underdeveloped" countries. Jill Stauffer's discussion of Truth and Reconciliation Commissions provides an effective illustration of how the institutions meant to improve the lives of people in positions of disadvantage can in fact perpetuate epistemic injustices. Anna Malavisi's work on Chagas Disease in Bolivia and Kristie Dotson's examination of Lorraine Code's work on Tanzania health care initiatives demonstrate clear cases of epistemically unjust practices happening on the ground in real development projects. Rajeev Bhargava raises another important dimension of epistemic injustice more generally with his discussion of epistemic frameworks and colonialism's domination through the repression of alternative ways of being in the world. Through discussing these examples, I argue that a proper account of epistemic injustice is essential to effectively pursuing the goal of global development: improving the lives of the most disadvantaged, marginalized and oppressed people in the world.

Following Malavisi's lead, I will be using the term "global development" instead of "international development" to describe the "system of ideas, policies, institutions and individuals all supposedly concerned with the improvement of the living conditions of 
those populations living in disadvantaged conditions" (Malavisi, 2). Among those populations living in disadvantaged conditions, I include not just countries that are commonly designated "underdeveloped" but also people in conditions of deprivation and oppression located in so-called "developed" countries. Specifically, I include in the concerns of global development the disadvantaged conditions of Indigenous peoples, homelessness and extreme poverty anywhere in the world. I find "global development" to be a more encompassing term that allows the inclusion of issues at home as well as abroad.

\subsection{The Idea of Development}

The very concept of development is contentious, and has evolved over the years. There is no one single definition of the term, although it has been used in a number of ways over the past several decades. Denis Goulet claims that in the early years of "development" as a discipline, it "was generally defined as a straightforward economic process" (2006, xxxi). However, it has come to mean a great deal more than that. Approaches to development have expanded beyond just focusing on empirically measurable economic factors, and now pay more attention to "human capabilities" and to other components of individuals' well-being. According to Goulet, development is widely acknowledged as “a process involving essential value changes in the conception any society has of 'the good life,' of the relation between 'goods' and the 'good,' and of the stances it adopts toward nature" (xxxi). This will involve not only economic change but "technological, social, political, and especially value change" (xvii). In short, the attention should be on human development, not just economic development. I will be using this concept of 
development, with the addition that the goal of that change is to improve the well-being2 of people.

It should be evident here why global development provides a context that is particularly vulnerable to epistemic injustice. When certain groups of people employ their values, relationships, and beliefs in their efforts to cause change through development, there is an increased likelihood that the values, beliefs, experiences, and knowledge of the people who are the targets of development will be discredited, marginalized, silenced or not heard. This is particularly the case when the structural injustices caused by colonialism and often reinforced by more recent global political and economic systems are the assumed background of development efforts. The power inequalities behind those who tend to be the ones implementing "development" and those who are "underdeveloped" have tended to follow the global power inequalities that have historically led to domination and oppression.

Escobar, a post-development theorist, is very critical of what he calls the "discourse of development" $(1995,6)$. He argues that "development" has led to ineffective practices that not only did not improve but actually worsened the conditions in these situations (5). He also argues that the discourse on development has served as a form of domination, a type of "colonization of reality", which allowed the West to "exercise control over the Third World" (9). He claims that "development was- and continues to be for the most part- a top-down, ethnocentric, and technocratic approach,

2 I have chosen to use the term "well-being" because I feel that it incorporates a wider range of different interpretations of what "development" means. I wish to include in this concept not only economic well-being but also ideas such as Serene Khader's concept of "flourishing" and the different conceptualizations of "capabilities" from Amartya Sen and Martha Nussbaum, among others. 
which treated people and cultures as abstract concepts, statistical figures to be moved up and down in charts of 'progress"' (44). As a result, he argues that the concept of "development" is inextricably bound up in conditions of colonialism, imperialism, unequal power and domination. As a result, he thinks it should be abandoned altogether (11).

I do not necessarily agree with Escobar's denunciation of development, as a discourse or as a practice. However, Escobar rightly points out that Western scholars have ignored "the sizable and impassioned critical literature by Third World intellectuals on... development" (15). Susan Babbitt points out something similar when she says that there are plenty of alternatives to liberalism, "but they are mostly from the south" and so they are discredited (forthcoming 2017, 266). An epistemic injustice occurs when these critical views of liberalism's version of development, coming from the people who experience it and have a particularly valuable understanding of it, are marginalized and discredited. This should be a great source of concern for development theorists, because if the people that development is meant to help are saying that it is not working and is in fact only perpetuating their deprivation, oppression, and domination it is a significant practical and epistemic problem. These critical voices should be listened to and engaged with, in order to determine the best way forward in helping to improve the well-being of people in these situations, regardless of whether the term "development" should be applied. Development, as a concept, may be vulnerable to imposing epistemic injustices, but these injustices are not essential to the concept of development. The goals of development can be pursued in an epistemically just way. 


\subsection{Institutions}

One of the other ways in which development theory and practice can be epistemically unjust is through the policies used to determine the practical means of achieving "development", and through the institutions that shape those policies. There are a wide variety of these institutions and policies, which form a rather hierarchical system through which global development is carried out around the globe (Malavisi 2015, 5). Within this system are included various governments, multilateral agencies, non-governmental organizations (NGOs), corporations, social movements, community organizations, and individual people. All play roles in how development policies are shaped and carried out, and all relate to each other in a variety of different ways. In these roles and relationships, there is plenty of room for epistemic injustices to arise, particularly with the influence of power and inequality that comes in any hierarchy. Code's ecological thinking, discussed in Chapter 3, helps to draw out the complexities of the relationships that develop between these different elements in the hierarchy.

The hierarchical structure of this system, where those with greater resources for addressing the problems of global development have the least direct access to the experiences of the people they are meant to help, means that the relationships between the different institutions are often deeply entrenched with dynamics of power and domination (3). The power relations that are created, particularly between poor countries (usually "Third World" and underdeveloped) and rich countries (usually "the West" and developed), can often lead to an attitude of superiority and authority on the part of the rich countries and those institutions and professionals based in such countries. Institutions and professionals from the West are seen as authoritative and more knowledgeable, and 
as a result their paradigms of development are frequently assumed to be correct (49).

Professionals from multilateral agencies such as the World Bank (WB) and International Monetary Fund (IMF) are seen as "the only source for expert knowledge on development" and are given "higher epistemic authority and hence, credibility than the knowledge of others" (60). However, most often the knowledge produced by these economists and development professionals "is completely divorced from the reality of poorer people in less advantaged countries" (61).

Malavisi argues that this is demonstrated when "development professionals visit poorer countries for a few days and believe they 'understand' the situation of the others, and therefore base their decisions on this experience" (118). Such cases illustrate a credibility excess attributed to development professionals and institutions from the West, which will inevitably impact the relationship that these professionals and institutions have with those they are trying to help. It is likely that the impact will be a credibility deficit attributed to the local people, those who are considered "underdeveloped" and therefore less knowledgeable. This is why alternative conceptions of development embedded in local cultures and experiences are frequently marginalized and given little credibility. The credibility excess given to "the West" results in a corresponding credibility deficit given to those who are not part of it.

When this happens, the marginalization of the beliefs and experiences of local people leads to an inability on the part of practitioners and policy makers to truly understand the realities of those they label "underdeveloped", and sometimes even the marginalized themselves will be unable to understand their experiences (McCollum 2012, 191). This is because marginalized people are confronted with interpretations that don't 
fit their experience, and practitioners and policy makers are confronted with experiences that don't fit their interpretations. The result is a clear epistemic injustice, where unequal credibility is given to those seen as authoritative (Western development professionals) and those seen as ignorant (the "underdeveloped" people). Some problems go completely unrecognized, while the dominant institutions default to "simply and easily applied metrics" (196) to make the problems that they do recognize fit their understanding of the world.

The result is that structural and historical causes of deprivation and oppression (such as colonialism, failed structural adjustment programs, inequalities in the global economic order, and many others) tend to be ignored in favour of simple explanations that do not implicate dominant countries in the problems of the "underdeveloped" ones. Code's ecological thinking provides further insight into the importance of these complex contextual details that form the environment in which epistemic injustices arise. Looking at the relationships that develop in such contexts and at the complex multiplicity of the selves they create (discussed in Chapter 3) we can begin to see the insidious effects of the dominance of perspectives that have shaped global institutions.

The lack of accountability that can result from the hierarchical structure of the institutions of global development will also sometimes perpetuate epistemic injustice. Because many NGOs and other agencies tailor their policies to "comply with donors' strategies" (Malavisi 2015, 54), they may ignore the realities of particular contexts that go counter to those strategies. In such situations, where an NGO chooses to discredit the concerns of community members or to fail to consult with them altogether because the testimony they would receive runs counter to what their donors want them to do, there is 
an obvious cognitive bias at play. The development institutions in question are motivated to remain ignorant of the real issues in the community in question, in order to maintain deniability in following the interests of their donors rather than those they are trying to help. In such a case, the institution prioritizing their relationship to their donors over their relationship to the local people causes an epistemic injustice.

There are other ways in which epistemic injustices can manifest in the policies and structures of development institutions, which cannot all be discussed in detail here. However, it is important to note that the institutions of development form the background structures that produce knowledge about what development is, how it should be carried out, who it applies to, and who can implement it. As a result, they will be a significant locus of epistemic injustices that must be addressed in order for global development to achieve its goal of improving human lives. Even the most well-intentioned institutions can cause epistemic injustice.

\subsubsection{The Institution of the Truth Commission}

Truth commissions, meant to address the legacies of unjust and oppressive systems such as apartheid in South Africa or residential schools in Canada, are one such type of institution that is well-intentioned but which can still miss addressing harms that emerge from epistemic injustice. Although truth commissions may not usually be considered global development institutions, I think they play a very important role in development practice and in achieving (or failing to achieve) the goal of improving peoples' wellbeing. More often than not, truth commissions are set up in countries where systematic injustices and great trauma have been perpetrated against a particular social group, usually an oppressed one. If global development is, among other things, a social, 
political, and value change then the institution of the truth commission plays an important role in certain contexts of development.

Truth commissions are meant to provide a venue in which the victims of atrocities can tell their stories. While legal trials focus primarily on trying to "mete out judgment about wrongs inflicted" (Stauffer 2015, 41) and often only pay attention to the victims when they are needed to build the case, truth commissions try to "build a more comprehensive narrative of harms" that focus on the victims in their contexts (45). Both legal trials and truth commissions can have an important role to play in helping to address the kinds of harms and wrongs that are among the most destructive barriers to well-being. However, truth commissions are a particularly powerful tool when it comes to ensuring that the voices of those who suffered are heard. They have the potential to draw attention to the relationship between systemic wrongs of violence, poverty, oppression, and silencing, on the one hand, and the harms done to individuals, on the other (45). Unfortunately, even in the use of this tool that is meant to amplify the voices of the oppressed epistemic injustice often occurs. Attempts at "giving voice to the voiceless" do not necessarily ensure that they are actually heard (55).

Stauffer discusses truth commissions as one way to demonstrate the causes and harms of "ethical loneliness", which I discussed in section 1.1. with respect to silencing. Ethical loneliness occurs when someone's experience of injustice is compounded by a feeling of isolation and abandonment by humanity (1) as a result of not being heard. Stauffer describes multiple instances where those that truth commissions are meant to help experience ethical loneliness as a direct result of epistemic injustices that occur during those proceedings. 
The stories of apartheid in South Africa had been told for many years in many diverse ways before the implementation of the South African TRC. However, the TRC was beneficial as "a new structure for speaking combined with a guaranteed audience and a non-retributive but still institutionalized way of dealing with harm" (83). Unfortunately, within that new structure the categories and labels used by the TRC restricted the understandings that could be obtained by the commission and influenced the credibility given to people's testimony in ways that turned out to be harmful. For example, only 'grave violations' were recognized as falling within the purview of the TRC, and these were restricted to "killing, torture, severe ill-treatment, and abduction" (47). This meant that those who suffered harms caused by many of the laws passed by the apartheid government (such as forced removals, hunger, poverty, and lack of health care) were not recognized as victims under the TRC (47). The TRC also ignored important distinctions, such as "the difference between direct and indirect perpetrators", and did not recognize the possibility of group perpetrators (47). These narrow definitions meant that the complexities of many of the experiences people had under the apartheid regime could not be fully recognized by the TRC.

Lugones' logic of curdling and Code's ecological thinking, discussed in Chapter 3 as informing my new approach to epistemic injustice, provide an alternative way of implementing the institution of the TRC that could give a more complex view of the situation. This alternative approach draws out the messy relationships between perpetrators and victims, and also within individuals between conflicting aspects of their selves that are considered perpetrator or victim. It also draws out the different degrees to which similar incidents may impact different individuals in different ways, making a 
lesser harm to one person a grave violation for another. The specific environment of each case will have a further impact on the meaning of that scenario for those impacted, both directly and indirectly.

To illustrate, Stauffer discusses Yazir Henry, a former member of the armed wing of the African National Congress (ANC) who was captured, tortured, and forced to give up a friend's location to his captors. That friend was then killed. Upon his release, Henry's friends and allies suspected he was a traitor, which led to him being ostracized and fearing for his life (94-95). Stauffer uses Henry's case to show how the strict separation of victim and perpetrator prevents an accurate understanding of his experiences (95). Henry was a victim who was captured and tortured. However, in the eyes of other victims, he also perpetrated a grave violation in betraying his friend to those who eventually killed that friend. This illustrates the problems of categorization discussed in Chapters 2 and 3, where giving strict definitions such as "grave violations", "victim", and "perpetrator" results in the nuances of peoples' experiences and very selves being lost when they fall outside of these definitions. Lugones' analysis of the multiplicity of the self, discussed in section 3.3, helps to shows why this is the case.

Categorization also misses how different events can have different significance for different people. It may be the case that for some people it would be the ostracization from friends and allies, leading to a loss of relationships, rather than the torture that would count as the worst "grave violation". Ecological thinking will help us understand why, showing how a person's environment and culture will help to shape their values and meanings. The logic of curdling will also provide insight into how the strict structures delineated by a truth commission can leave gaps in understanding and marginalize certain 
experiences, causing an epistemic injustice. White ignorance, discussed in section 3.1, is an example of what falls through those gaps and causes epistemic injustice that is particularly relevant to the case of the South African TRC.

There are also examples of epistemic injustices that are committed directly against the testifiers during the institutionalized proceedings of truth commissions. Stauffer focuses on cases where interviewers, either intentionally or unintentionally, "encourage certain kinds of testimony and discourage others" (73) when testifiers are telling their stories. As discussed in Chapter 3 (in the context of white ignorance), there are many reasons why a hearer may not want to hear the story that the testifier wants to tell (74). This is particularly the case in truth commissions, where part of the purpose of the commission is to pave the way for a country or society to move past grave injustices and atrocities in a way that allows peaceful coexistence between groups that were previously doing serious harm to one another. There is a great incentive to ease the way toward achieving that goal by only hearing what the commission wants to hear. Even if that kind of selective listening does not occur, sometimes the previous marginalization of certain experiences and perspectives makes it impossible for them to be heard well simply because they are not understood. In either case, an epistemic injustice is occurring that prevents the kind of understanding and even recognition of certain truths of the past that are necessary for effective and genuine reconciliation. A similar kind of selective hearing also happens in Robinson's case in To Kill a Mockingbird, where even Finch's advocacy coming from the position of privilege of a white man is not heard because it goes counter to assumptions that the society is invested in, as discussed in section 2.2.4. 
Another example Stauffer discusses in her account of ethical loneliness is the story of Yvonne Khutwane, who testified to the South African TRC about a variety of forms of violation that she had experienced during the "struggles around and against apartheid" (82). While the TRC "guided" her testimony to foreground sexual violence as the central violation (82), her original written testimony and her oral testimony to the TRC resisted making it a story of rape. Instead, she was focused on the humiliation of being "dehumanized by young people the age of her children" (82). Because she came from a culture where elders are respected, it was "the violation of that respect, when added to the other losses and abuses she describes" that was the most harmful for her (83). There is a clear gap in understanding between the commissioner's preconceptions about what is most important to Khutwane's story and what Khutwane herself found to be most important to her experience. This gap resulted in Khutwane's account, the way she wanted to tell her story, being given less credibility than the story that came out and was shaped by the commissioners "guiding". This in itself is an epistemic injustice. However, Khutwane experienced a further epistemic injustice when she lost control over her own narrative of her experience. News media and academic writers took up her story, but they took up the version that had been shaped by the commissioners, and "effectively reduced how Khutwane's testimony would be heard outside the TRC to a narrative of sexual violence" (83). The way Khutwane chose to tell her story should be seen as "an assertion of agency, a form of resistance to the expected narrative form, and an attempt to reclaim her self by means of her own story" (82). However, her story is coopted, first by the commission and then by the news media and academia. This not only silenced her but in a sense also violated her all over again, disrespecting her agency. It undermined her 
credibility and marginalized her understanding of her experience, causing an epistemic injustice.

Stauffer argues that there have been attempts to mitigate and eliminate these kinds of epistemic injustices in the processes of truth commissions. The incorporation of victim advocates into the process may help mitigate epistemic injustice by mediating "preconceived ideas of what an average victim would want or need- ideas formed without consulting with victims and without considering the wide range of different needs and desires different victims might have" (61). These processes would hopefully provide consultation with individual victims about their needs and desires that is often missing. Inclusion (62), careful listening (63), and reasonable expectations (63) will also play important roles in ensuring that ethical loneliness and epistemic injustice are avoided. However, the only reliable way to mitigate epistemic injustice is to be aware that it is a real and present risk, regardless of well-intentioned individuals or commissions. Only with conscious efforts to mitigate it can epistemic injustice be avoided, and only then can processes to improve well-being become effective.

\subsection{On the Ground Practice}

The practice of global development, on the ground working with the actual people that development policies and institutions are meant to help, is particularly vulnerable to epistemic injustices. This is because in such practice, it is often foreign (usually Western) practitioners who come into a community to which they do not belong and try to implement the policies developed in institutions far away. It is also because in any relationship between the members of dominant and marginalized social groups there is risk that epistemic injustice could arise from the power imbalances present in those 
relationships. To exemplify how the relationships created in development practice on the ground are particularly vulnerable to epistemic injustice, I will discuss two examples of health focused development initiatives whose effectiveness, justice, and ethics are compromised by epistemic injustices. The first example looks at how hermeneutical marginalization and prejudiced credibility assessments result in the neglect of those affected by Chagas disease in Bolivia. The second example, of Tanzanian health care initiatives, will illustrate how even policies intended to decrease the epistemic injustice of development practice can still fail to treat marginalized peoples as full epistemic agents.

\subsubsection{Chagas Disease}

Malavisi discusses the Neglected Tropical Disease (NTD) Chagas disease, a fatal disease caused by the bite of a bug3 known locally as the vinchuca, which mainly affects areas of Latin America. Malavisi's focus is Bolivia, having herself done development work there. The disease often goes undiagnosed because it can be asymptomatic for years, and so the connection to the vinchuca is rarely made by those suffering from it (Malavisi 2015, 46). The bugs live in the cracks of mud brick homes and on animals such as guinea-pigs and chickens (47). As a result of the carrier's chosen habitat, the disease "mostly affects poor, often illiterate campesinos or indigenous peasants who often live in precarious, overcrowded housing and rely on subsistence farming" (7).

The poverty of those most affected by Chagas disease makes preventative measures, such as improving housing, difficult to implement for economic reasons (52).

3 Malavisi calls the vinchuca a beetle (order Coleoptera), however is in fact a true bug of the order Hemiptera. It is also not the bite itself that transmits the disease, but instead a parasite that is transferred from the bug to the human when the bug's defecation enters the wound caused by the bite (World Health Organization, Media Center, Fact Sheet, Chagas Disease [American Trypanosomiasis], updated March 2016) 
Because of the lack of symptoms, the bite of the vinchuca is not usually connected to the patient's death. Thus, it is very difficult to convince people that such measures are worth the cost. Social factors also get in the way of effectively addressing the disease, as the presence of many vinchucas in a home is seen as a symbol of the high fertility of the resident animals (52). This makes the campesinos even less willing to take measures to remove the insects. Health campaigns used by global health organizations to educate people about Chagas disease often simply "consist of 'telling' people that Chagas disease was caused by a vinchuca and that they need to get rid of them" (57). This strategy does not take into account the knowledge, beliefs, and values of the campesinos, and instead treats them as less than full epistemic agents who need to be informed (57). Through failing to acknowledge their alternative hermeneutical resources that may provide alternative understandings, these health campaigns discredit the knowledge that those who have experienced Chagas disease have about their experiences with the disease and their understandings of what causes it and how it should be addressed (62). This credibility deficit and the accompanying hermeneutical marginalization undermines the effectiveness of attempts to address the disease.

Further problems arise from Chagas disease being treated as a clinical health problem that just needs to be addressed by pharmaceutical companies (47), rather than being approached with a more community health oriented perspective. Finding a pill or vaccine is seen to be the solution in the clinical approach. However, this is insufficient in the case of Chagas disease because it ignores the social, political, economic and cultural conditions of the particular contexts where the disease manifests which may pose a barrier to it being effectively addressed (47). Knowledge about those conditions, such as 
the poverty that makes it difficult for the Bolivian campesinos to improve their homes, should be gained directly from the people themselves rather than from the assumptions of practitioners.

The neglect that results from that poverty leads to the marginalization of those suffering from Chagas disease from the production of hermeneutical resources used to understand the disease by the dominant global health organizations (119). The experiences of "poor indigenous peasants" are rarely counted as credible or relevant knowledge when it comes to concerns like health, and so their knowledge is discredited and silenced. This dismissive attitude is a direct result of the excess of credibility give to institutions such as global health NGOs, pharmaceutical companies, and professional scientists. They are taken to be the exclusive authorities on the Chagas disease, reflecting the comparative nature of credibility discussed in section 4.4. of this chapter. Moreover, these authoritative institutions are dominated by individuals who have never experienced the material, economic, social and cultural conditions that lead to being infected by Chagas disease, and who know little about the circumstances of those who have had those experiences. Although these dominant sources of knowledge do have a great deal of justified credibility and knowledge when it comes to the medical and biological details of the disease and to how pharmaceuticals can be used to treat it, they do not tell the whole story. They cannot adequately understand the roles that social, economic, cultural, and political factors play in how Chagas disease plays out in Bolivian communities. Knowledge of those other factors, which must be drawn from the experiences and knowledge of the campesinos, are necessary to create effective measures for the prevention, treatment, and cure of the disease. 
Effectively addressing Chagas disease will require knowledge embedded in context, not abstracted away (69). It will also need to use the resources provided by social and cultural beliefs, the resources available, and the people's knowledge about their everyday lives to cooperatively build an understanding of how best to address the disease in each particular context. This understanding will be based on the relationships between the vinchucas and the campesinos, the campesinos and their animals, and the members of the community in question. It also needs to incorporate the relationships between the members of the community and the development practitioners, the development practitioners and the NGOs they work for, the NGOs and Western countries, the NGOs and the pharmaceutical industry, and the pharmaceutical industry and the scientists that do their research and development. An effective method of treating the disease must look at all of these relationships and different dimensions of the context in order to determine the most effective methods of treatment and prevention.

The relevant factors become evident when a relationship focused approach to development is used, and this is the insight that Code's ecological thinking gives us. To understand a phenomenon such as epistemic injustice in cases of Chagas disease, all of the complexity of the context must be considered. This can only be achieved if global health organizations incorporate the knowledge of those experiencing Chagas disease, with the intent of gathering alternative hermeneutical resources specific to each context and the network of relationships people are in, and of giving due credibility to the experiences of those living with the disease. The importance of this kind of understanding, embedded in the context of particular communities, will be discussed further in the case of Cuba in Chapter 5. 
Although Malavisi starts to acknowledge the importance of incorporating the context and knowledge of the campesinos into attempts to address Chagas disease, the true complexity of the situation and of the needed analysis still does not come through in her discussion. She does not go far enough to show the extent to which the hermeneutical gap exists. Yet, the voices of the Bolivian campesinos are still missing from her account, as much of what she uses to support her argument about what the dominant global health organizations miss in Chagas disease come from her own experiences as a development practitioner. Although this is a better position from which to gain knowledge about the disease in its context than a policy maker who has never been to Bolivia, it still does not provide the perspective of the Bolivians themselves.

\subsubsection{Tanzanian Health Care Initiatives}

Further complexities of how epistemic injustice arises in development practice are revealed in an exchange between Code and Dotson, who look at the impact of participatory approaches to health care initiatives in Tanzania. Code argues that Tanzanian health care was usually approached in a "vertical" way, applying information with little consideration of whether it was actually relevant to a particular population or location $(2008,41)$. This vertical approach imposed on the subjects both credibility deficits (37) and hermeneutical marginalization (41). It objectified the local Tanzanians, failing to treat them as "presumptively credible in the exchanges in which they are sought out, and listened to, as conveyors of information/knowledge" (37). This objectification left out important aspects of their experiences of disease and of health, leading to ineffective attempts to address health care problems, and undermining their agency. 
Code's analysis reveals that the vertical approach in Tanzania encountered similar problems to those Malavisi identifies in the case of Chagas disease.

However, Code then moves beyond Malavisi's analysis. She discusses an alternative approach that was introduced in the 1990s as part of project funded by the Canadian International Development Research Center (35). The project used what she calls the syndromic approach (42), which Code argues treats the subjects as informants rather than sources of information (41). In the syndromic approach, "local, trained interviewers" interview families after the death of a sick patient, then send the information to a team of doctors. The information is then analyzed to give an accurate idea of local medical needs, particularly what health problems tended to overlap or coexist in certain areas (40). This approach gathers its information directly from the people, and ensures that contextual data is included for understanding how disease arises in each particular place based on the actual testimony of the people in that place. At first glance, then, it seems to effectively counter the problems of a "vertical" approach and to treat the subjects as informants rather than sources of information. Code reports the success of the new approach, claiming that "the results have been impressive" (42). However, Dotson cautions that although this shows "effective epistemic practices" (Dotson 2008, 57), questions of epistemic justice remain in doubt.

These effective epistemic practices, where the knowledge gained has resulted directly in practical successes (such as reduced mortality rates) are valuable. However, effective epistemic practices are not necessarily just epistemic practices. Dotson argues that simply because the epistemic practices are effective does not ensure that the Tanzanians being interviewed are being treated as full epistemic agents or informants, 
rather than mere sources of information (58). This is because it is possible for the health practitioners interviewing local Tanzanians to get accurate information (making the interviews an effective epistemic practice) while ignoring the beliefs, values, and knowledge of the interviewees. These beliefs and values, and the knowledge and understanding intertwined with them, must be considered if the interviewees are to be treated in an epistemically just manner as full epistemic agents and knowers.

Dotson argues that if someone who is interviewed for information "can give more information verbally than she/he is actually aware is relevant to the interviewer and is open enough to give that information when interviewed", then they are being treated as a source of information not an informant (59). In other words, an interviewee is treated as a source of information and not an informant if the interviewer reads something different from the interviewee than the interviewee intentionally told them. In such cases, even though the person being interviewed is being allowed to speak and is being asked for information, the interviewer is not fully engaging with them in the kind of collaborative reciprocal exchange that is the mark of treating someone as a full epistemic agent. The interviewer is failing to engage with the hermeneutical resources that the person being interviewed is using, and is instead imposing their own interpretation on the information being given. If the interviewer is gaining different information than the interviewee meant to provide, shaped by their hermeneutical resources rather than that of the interviewee, it is the interviewer and not the person providing the information that is choosing what information is credible and relevant. As a result, the interviewee is still being treated as a source of information rather than as an informant who fully knows and owns what they are telling the interviewer. 
I agree with Dotson that Code's account misses something by focusing on the effectiveness of the epistemic practices being used in the Tanzanian case. However, Dotson's analysis also does not go far enough. While she is correct that interviews do not guarantee that local people are being treated as informants, she does not discuss what is likely to be missed in these interviews that leads to the interviewees being treated like sources of information. Specifically, she doesn't talk about the relationships of the Tanzanian locals and the content of their knowledge. The reason that the interviews are insufficient to ensure an epistemically just exchange is because the information that the local Tanzanians have about their health cannot be isolated from the hermeneutical resources and epistemic frameworks which are informing them as they exist in their context. Code does bring ecological thinking into her analysis, and demonstrates the need to avoid colonial and racist stereotypes about local people, such as that it is ignorance and the local faith in traditional care that lead to poor health care outcomes (Code 2008, 41). However, it seems that even in the syndromic approach, the Tanzanians are not being engaged as knowers with alternative hermeneutical resources. Health initiatives and development practitioners need to engage with and understand these alternative hermeneutical resources in order for the epistemic practices being enacted to be just as well as effective, and for the subjects to be treated as full epistemic agents.

Part of what appears to be more epistemically just about the syndromic approach, as opposed to the vertical approach, is that it uses local people trained as interviewers to gather the needed information. This may be another way that Code can claim this approach treats local Tanzanians as informants rather than sources of information (60). Incorporating local people as the collectors of information would, it seems, help to 
minimize the hermeneutical marginalization that the "vertical" approach entrenches (60), and it is likely that local interviewers would give their interviewees greater credibility than an outside practitioner would give them. Both of these factors would reduce the epistemic injustice occurring in this case.

Dotson argues, however, that if the training the interviewers go through "involves 'Western sanctioned' evidence-collection procedures" then the new approach only serves to set up "a class of authorized 'speakers for the people" that "translate Tanzanian peoples' descriptions into a type of collective understanding Tanzanian peoples neither contribute to, nor play a part in instituting" (61). This is reminiscent of how TRCs restrict the meanings and understandings of those trying to tell their stories within those institutions, as discussed in section 4.2. In other words, using local people trained in Western methods does little more to prevent hermeneutical marginalization and reduced credibility than using a Westerner would. Although the interviewers are local and not part of the dominant social group (in this case citizens of Western countries), the epistemic authority is not. The interviewers are just conduits for the imposition of certain frameworks of knowledge in the same top down manner Code is trying to avoid.

In this argument, Dotson shows how because the syndromic approach relies on dominant hermeneutical resources and Western methodologies, it excludes the alternative hermeneutical resources of local peoples (including the interviewers) that stem from frameworks of knowing tied to their culture and history. The only way the information they collect can impact the health initiatives implemented by global development organizations is if that information is shaped into a form recognizable to the dominant hermeneutical resources. In this process, that knowledge is rendered unrecognizable to 
those whose experiences it is supposed to represent. Yet, if the Tanzanian interviewers did not use the methods viewed as authoritative by the dominant hermeneutical resources and instead used the alternative resources of the local community, they would not be seen as credible. The local Tanzanian interviewers are in a double-bind. Either they are seen as credible by dominant institutions and thereby perpetuate the marginalization of their own knowledge frameworks, or they will be unable to gain credibility for their knowledge in the institutions with the power to address the health care needs of their communities and thereby the people themselves will be deprived and marginalized. Either way, an epistemic injustice is occurring.

Here too, Dotson still falls short of what is needed to treat the subjects she is talking about as full epistemic agents. Her analysis gives little indication of the content of the alternative hermeneutical resources that she claims the local Tanzanian people are drawing on. She simply assumes that their understandings would be different, without articulating what is different about them. In doing so, she is treating them as sources of information, assuming difference without investigating the actual knowledge and understandings of the people she is discussing. Although it may be a justified assumption, relying on it falls short of just epistemic practice. It can be said that Malavisi also fails to reveal what Bolivians think of the attempts that have been made to address Chagas disease. In both cases, in addition to assuming differences in epistemic frameworks and hermeneutical resources, the authors fail to investigate what the people themselves think of the situation. How they understand their position, and the knowledge they have about its benefits and weaknesses, are critical to understanding the degree to which epistemic injustice is present in these initiatives. To fully engage them as epistemic agents, their 
voices need to be heard. Hearing involves awareness of the relationships in which they interact and engage with others at all levels of the local, national, and international.

In these particular cases, what is needed to reduce chances of epistemic injustice is not just the shift from treating Tanzanians (and Bolivians) as sources of information to treating them as informants. It also requires a fundamental change in attitude toward Tanzanian peoples and their knowledge, one that seeks cooperative methods that recognize the agency of individuals and the importance of their knowledge and the resources they draw from. Furthermore, although the syndromic approach is epistemically effective in the sense that it may improve health care outcomes, as Code indicates, if the approach is epistemically unjust it will fail in other important areas that are the focus of global development, such as empowering the local people, enhancing their agency, and creating a sustainable improvement in their lives. The narrow focus on health care isolated from its relationship with other components of people's well-being will impede the effectiveness of the approach.

\subsection{Epistemic Frameworks}

One of the things missing from Malavisi, Code, and Dotson is an analysis of the epistemic frameworks that lay behind the knowledge of marginalized peoples. When these frameworks, along with the alternative hermeneutical resources (as discussed by Pohlhaus) that are usually created by them, are ignored epistemic injustice will inevitably follow. Most of the policies and practices discussed up until this point are based on restricted frameworks of understanding that adhere to particular paradigms included in the dominant hermeneutical resource of "developed" countries. Stauffer's analysis of TRCs brings the primacy of dominant epistemic frameworks to the fore, and 
demonstrates its practical harms. Alternative frameworks and alternative hermeneutical resources are given less credibility than the dominant ones, and are marginalized from the institutions that produce knowledge and understanding about what "development" means and should entail. Global development policies and practices are then based on that restricted view of what constitutes knowledge. This insight will be critical for my analysis of the case of Cuba, which I will provide in Chapter 5.

In the context of India, Bhargava discusses how in the process of colonization Britain succeeded in marginalizing and discrediting entire frameworks of understanding, with lasting effects. He defines an "epistemic framework" as "a historically generated, collectively sustained system of meanings and significance, by reference to which a group understands and evaluates its individual and collective life" (Bhargava 2013, 414) and argues that such frameworks have an "inextricable link to individual and collective identity" (414). According to Bhargava, the loss of access to a group's epistemic framework will happen when "the basic epistemic forms of a group are altered by the arbitrary or deliberate actions of another powerful or dominant group" (414), making it impossible for the members of that group to access the meanings and understandings that were tied to that epistemic framework. Sustaining an epistemic framework requires that there be members of the group with the capacity to sustain it, and the marginalization or discrediting of the members of a group damages that capacity (414). The damage to the capacity to sustain epistemic frameworks will often happen "when one is simply born in a society where the dominant practices prevent one from having a secure knowledge of the historical and cultural traditions of one's own community" (414). It will also happen when the epistemic frameworks are accessible and known, but rejected as worthless 
(414). This is what often happens to alternative hermeneutical resources. As discussed in Chapter 2, alternative hermeneutical resources are the shared understandings that are produced within marginalized groups, used for understanding experiences that may not be shared with the dominant group and which may be excluded from the dominant hermeneutical resource. This has been one of the many challenges facing Cuba, where attempts to sustain an alternative way of understanding the world drawn from the philosophy of José Martí and Che Guevara have faced a great deal of adversity as outside influences try to discredit and eliminate it.

During colonization, establishing one country's superiority over another requires conquering "not only the land and goods of the colonized by also their culture and minds" (414). This relationship of power results in the destruction of the local capacity to sustain alternative epistemic frameworks that are traditional to the people being colonized, and in that process "the concepts and categories by which a people understand themselves and their world are replaced or adversely affected by the concepts and categories of the colonizers" (414). Such destruction has happened in India, the focus of Bhargava's discussion; in Canada's residential schools, discussed in section 1.1; and in Cuba, which I will discuss in the final chapter of this project. This is an intentional epistemic injustice, an attempt to silence any sort of opposition or alternative to the epistemic framework represented by the dominant hermeneutical resources wielded by the colonizers. According to Bhargava, the result is not only depriving the colonized of an understanding of their own intellectual traditions and epistemic frameworks, but also impeding their ability to have their own understanding of the "new knowledge received from Europeans" (415). This is happening in the case of Chagas disease in Bolivia 
(section 4.3.1) and Tanzanian health care (section 4.3.2), and it is why Malavisi, Dotson, and Code's lack of attention to the content of epistemic frameworks is a problem. In all of these cases, the knowledge of the colonized is distorted. It becomes derivative of European epistemic frameworks (415), which warp both the indigenous hermeneutical resources that shaped their way of being in the world and the experiences that they are trying to understand. The result reinforces the colonizers confidence that they are superior, and further marginalizes and discredits the colonized.

Bhargava does not think that the solution is to reject the dominant frameworks (416). They are important for communicating with the dominant groups, which hold a great deal of power on the global stage, and they have had an important influence on the history of colonized nations and how their cultures have changed since colonization. Bhargava points out that colonized countries are also "fully intermingled with the specificity of western practices; worse, they possess a deep imprint of western domination and hegemony" (416). This is why taking a structural approach that pays attention to relationships at all levels, as was discussed in Chapter 2, is necessary for understanding what is happening in cases of epistemic injustice. Such attention will show how structures put in place by colonization continue to lead to epistemically unjust outcomes.

The result of the deep connection between the epistemic frameworks of the colonizers and the contemporary cultures of the colonized is that "to non-western people, western thought is both recognizably their own and alien" (416). The dominant epistemic frameworks cannot be ignored, but they also cannot be separated from "their own imperial imprint" (416). In fact, for people in countries that were colonized, such as India 
and Cuba, "the very assumptions and presuppositions underlying [their] enquiries into [their] own world are shot through with categories derived from western experience" (416). Although western categories are inadequate for understanding the experiences of the colonized, they may have something to contribute to that understanding. The insights of Lugones' logic of curdling, discussed in Chapter 3, are important for drawing out why that is the case. The logic of curdling allows the colonized to recognize the influence of the colonizers' epistemic frameworks on their own understandings and analysis, while at the same time retaining a degree of difference from them. Through the lens of curdling, they can recognize these frameworks as both their own and not their own. The logic of purity, on which the "categories derived from western experience" are built, does not allow that kind of dual vision. There is likely no going back to the "original epistemic frameworks" (417) for groups that have been colonized. However, alternatives to the dominant hermeneutical resource and dominant epistemic frameworks can be created as the available resources are reinvented and rejuvenated through the cooperative and collaborative efforts of just epistemic practices.

Stauffer expresses an additional concern about the dominant epistemic framework. Unlike Bhargava, who focuses on the colonial heritage of the dominant framework, Stauffer focuses on the inadequacy of liberal individualist understandings of autonomy and culpability when it comes to addressing harms that come from abandonment and lack of recognition (specifically ethical loneliness). She argues for an account of responsibility and acknowledgement that pays attention to how relationships between human beings result in them having a great deal of influence in shaping selves and worlds (Stauffer 2015, 110). She examines the important role that uptake from others 
and reciprocity plays in our self-understandings and our ability to act autonomously. An approach that treats the self as a completely individual unit, self-sufficient and isolated, is one that misses the role others play and fails to attain a lasting or meaningful understanding of what equality and autonomy mean and what is needed to achieve them (66).

The individualist approach denies that "you can be destroyed" by others, and insists that "you're responsible only for acts you intended to commit" (79). It views selfsufficiency as possible outside of social support (79). On a relational account this is not only inaccurate but incoherent. At many points in every human's life, other people are necessary in order for us to achieve our needs and ends. The relational approach defended by Hoagland and Medina in section 2.3.2., and Code in section 3.4., demonstrate some of the theories bringing this insight to the forefront. Not only is the isolated individual an inaccurate and incomplete view of human beings, but such an understanding can result in injustice (11), both epistemic and moral. Seeing responsibility as something belonging to an autonomous, individual self cannot capture the systemic causes and harms of issues like racism, gender inequality, poverty, and underdevelopment.

It is also necessary to point out that epistemic injustice, and particularly the marginalization and discrediting of certain frameworks, is not only imposed on the Global South by the Global North (or by "developed" on "underdeveloped" countries). There are "discrepancies, power differentials and marginalization that also occurs within the heterogeneity of the south" (Malavisi 2015, 103) and I would add, in the heterogeneity of the north. This is true even within particular social movements (105). For example, recent discussions about feminist organizations in the Global North point 
out how even within the women's movement there is exclusion of certain groups, such as women of color and transgender women, and certain frameworks of understanding, such as non-binary gender identities.

Another example can be seen in Western academia's treatment of epistemic frameworks that do not reflect liberal individualism, such as that proposed by Karl Marx. Susan Babbitt criticizes the academic abandonment of Marxist insights after the fall of the Soviet Union, pointing out how anything that speaks well of a philosophy associated with Marx is now ostracized from academic publication (Babbitt 2017). Much of the prejudice against Marxist philosophy, which leads to it being discredited and hermeneutically marginalized even within academia, seems to be the result of an unwillingness to face the challenge it poses to the dominant epistemic framework.

Malavisi, Code, Dotson, and Bhargava only begin to show how frameworks such as colonialism, imperialism, individualism, and capitalism have marginalized and discredited all other frameworks for understanding the world, often while at the same time entrenching patriarchal, racist, and otherwise discriminatory hermeneutical resources. In the next chapter, I will look at a particular example of an epistemic framework supporting an alternative view of humanity in the form of Guevara's "new man" of socialism. Guevara's approach, and the wider epistemic framework at the foundation of the Cuban Revolution, exemplify a marginalized world view that has been discredited not because of its content but because of prejudice.

\subsection{In Summary}

This chapter only gives a basic overview of the ways in which epistemic injustice can influence global development in theory and in practice. The work of Stauffer, Malavisi, 
Code, Dotson, and Bhargava serves to provide a snapshot of the ways in which prejudiced credibility judgments and the marginalization of certain hermeneutical resources and epistemic frameworks can impede attempts to improve the well-being of people around the world. Epistemic injustice is present in the very understanding of what development means, in the policies of institutions, in the practices of development on the ground, and in the frameworks of understanding that shape those policies and judgments. At each of these levels, it disrupts the understandings and knowledge that are necessary to wholly comprehend what is happening in "underdeveloped" countries and how the problems of "development" can best be addressed in an effective and just manner.

It is important to note that many of the sources I have drawn from for this chapter are from white women from Western countries. Although their analyses of the challenges faced in global development are very valuable, it would be preferable to incorporate more of the primary voices of the people most impacted by these contexts and the epistemic injustices that arise in them. Those voices don't come through, even in Dotson's critique. The fact that few such voices are readily available makes even more obvious the hermeneutical marginalization of people in underdeveloped countries whose philosophical works, and knowledge more generally, are often not available to westerners.

In the next chapter, I will discuss in greater depth the role played by colonialism, capitalism, and individualism in the dominant epistemic frameworks of global development. I will examine how Cuba's approach to education and health care, and the philosophy that has shaped it, has been rejected, ignored, marginalized, and discredited because of its association with an alternative epistemic framework that challenges the 
dominant one. In doing so, I will try as much as possible to put the voices of Cubans themselves at the forefront of the argument. I will argue that an epistemic injustice has been committed, as the dominant capitalist/liberal paradigm's fear about anything even slightly associated with communism has led to the complete dismissal, discrediting, and marginalization of the resources that the Cuban approach to education, health care, and humanity itself could provide for development in other parts of the world. 


\section{Chapter 5: A Cuban Case Study}

So far, this project has explored Miranda Fricker's theory of epistemic injustice and its flaws, while also developing a new approach to epistemic injustice and its application to global development. In Chapter 1, I provided a detailed summary of Fricker's account in Epistemic Injustice. In Chapter 2, I discussed a variety of critiques of that account, which all converge on the need to move away from categorizing the different types of epistemic injustice and toward an approach that focuses on the structural nature of injustice and the relationships that shape and influence it. In Chapter 3, I draw on María Lugones' work on the logic of curdling and the multiplicity of the self, as well as Lorraine Code's ecological thinking, to shape a new approach to epistemic injustice. In the previous chapter, I discussed some of the ways in which epistemic injustice may arise in the dominant understanding of development, the institutions of development, the practice of development on the ground, and the epistemic frameworks that exist in the background of it all.

In this final chapter, I seek to apply the lessons learned in the previous four chapters to the case of Cuba. I have chosen to focus on Cuba for this case study because I think that the country has something unique to contribute to a discussion of epistemic injustices related to global development in the real world. The revolutionary government of Cuba is explicitly trying to enact an alternative epistemic framework that challenges the one that dominates the world today. This framework is backed by philosophy that was developed for and by Latin Americans. It draws on a view of what it means to be human and to be developed that is drastically different than the liberal individualism that is often pushed by the institutions and theorists of global development as the only acceptable 
framework. As one of the last bastions of socialism left among the world's national governments, Cuba is often reviled as a communist dictatorship, a label that it has actively tried to dispute. The revolutionary government also actively resists the kind of colonial domination of epistemic frameworks that Rajeev Bhargava describes, and in the process has met further hermeneutical marginalization and lowered credibility. However, despite these epistemic challenges and a myriad of economic, social, and political barriers to reaching its goals, the country has made revolutionary gains in the areas of health care and education through an approach that reflects an alternative world view that itself shapes a new kind of relational self.

Health care and education are central to achieving the well-being that is the goal of global development, no matter how one understands "well-being”. Many international laws and conventions, as well as theories of development, focus on using health and education to push toward better outcomes in other areas of development. Because of its importance, the epistemic injustices that arise at the level of hermeneutical resources in these areas are particularly concerning. This makes the case of Cuba even more important to discuss. Because of the marginalization of its epistemic framework and the philosophy behind it, the contributions that Cuba can and has made in these areas of global development are not adequately or accurately considered by the global community. What emerges from Cuba's unique epistemic framework is a conviction that everyone has a right to health and education, and therefore the state must provide those services. Closely tied to this conviction is the belief that moral incentives are more important than monetary incentives, and so everyone is responsible for contributing what they can to the state to help carry out this responsibility. This is a drastically different way of looking at 
health and education than the one that is often used in global development, and reflects an epistemic framework and philosophy that actively challenges the hegemony of liberal individualism.

It should be mentioned from the outset that finding English translations of work done by Cuban intellectuals is difficult. Even the MDG country report for Cuba could only be found in Spanish. Most of the sources on Cuba that I discovered were coming from authors in Canada, the U.S.A., Norway, and Belgium. Those sources were sympathetic toward Cuba, and many of the authors had travelled to Cuba and worked extensively on the topic. However, they still reflected the bias of the dominant global attitude towards Cuba, and pay little attention to the philosophical background behind Cuba's policies or the alternative epistemic framework they reflect. I was only able to find three sources written by Cubans themselves on the topics of education and health care. Even when I asked a Cuban friend who is studying in Canada to recommend a source in English, the source she recommended was written by a Canadian academic (John M. Kirk) rather than a Cuban one. This is just anecdotal evidence, however the fact that it was extremely difficult to find Cuban sources in English using the resources of a Canadian university is worth noting. It should also be noted that the works of José Martí and Ernesto "Che" Guevara have only been translated into English relatively recently. Martí was writing in the late 1800s, however the translation I will be drawing on is from 2002. Although Guevara's work is much more recent, the sources I will be using were translated in 2002 and 2003.

Of course, I would not expect to find sources written in English by Cubans. To expect Cubans, or other non-English speaking people, to cater to English speakers would 
be arrogant and would perpetuate the marginalization of those who do not speak English. Some Cubans may be able to write in English, but choose not to out of respect for their own language and culture, resisting the domination of the English-speaking world. However, the lack of sources translated by English speakers also shows how few attempts have been made by people in the West to make such resources available. Furthermore, the lack of effort by those working in health, education, development theory and philosophy in the West to make these resources more accessible to the English-speaking world only furthers the marginalization of these alternatives from the dominant hermeneutical resources of the global power structure, and is itself an epistemic injustice. This chapter attempts to show that it is to the detriment of all who are concerned about injustice.

It should also be acknowledged that my defense of Cuba and its epistemic framework is controversial. As I argue in what follows, there are good reasons to tell a different story of Cuba than the one that is conventionally rehearsed. A degree of humility and openness is called for if we in the West are to adequately address the charges of colonizing epistemic frameworks that Bhargava has leveled against us, and of the kinds of epistemic injustice that we tend to be guilty of when we fail to acknowledge alternative hermeneutical resources. However, even if you do not agree with the need to embrace another way of looking at the world, there is something extremely valuable about Cuba's alternative epistemic framework from the point of view of global development. This is the case even if one is critical of the revolutionary government, and even if one chooses to focus on their alleged rights violations and the restrictions of "freedom" as it is understood in the dominant world view. Even by the standards of the 
West, the Cuban health and education systems have made great strides towards achieving the goals of global development as they are articulated by dominant global institutions. That at least should give the ideas behind those advances some credibility, and should make those who are part of the dominant framework give the alternative hermeneutical resources recognition and consideration. Even if one disagrees with the Cuban approach, mitigating epistemic injustice demands at the very least listening to what they have to say.

\subsection{A Brief Review of Cuban History}

The island of Cuba was colonized by the Spanish following Christopher Columbus' "discovery" of it in 1492 (Brenner et al. 2008, 2). What followed was a familiar story of colonization, with the elimination of most of the aboriginal peoples, the monopolization of trade by the colonizers, and general neglect of the needs of the people (2). Slavery, used to fuel the sugar plantations, shaped the population and ingrained a deep racial divide that manifested in structural inequalities that persisted through the centuries (3) and remain today (Fuente 2008, 316-325). After a lengthy struggle starting in 1868, Cuba officially achieved independence in 1903 as part of a bilateral peace treaty that the U.S. signed with Spain (Brenner et al. 2008, 5). However, that "independence" included a provision "which permitted U.S. unilateral intervention on the island" (5). In effect, U.S. imperialism and neocolonialism had replaced Spanish colonialism, and it "stifled Cuban development" (6). Although during this time "a limited form of democracy did emerge on the island", it was associated with corruption and domination through a great deal of U.S. interference (7). This eventually led to Fulgencio Bastita Zaldívar, who had been elected 
in 1940 and then lost power in 1944, overthrowing the constitutional government in 1952 and implementing a military dictatorship backed by the U.S. (7).

Throughout 1958, an armed revolution led by small group of fighters and backed by a great deal of popular support used guerrilla warfare tactics to overthrow Batista's regime, succeeding when Batista fled the country on December 31, 1958 (8). Among that small group of fighters was a doctor, Guevara, who would become a crucial philosophical figure in the revolution and a lawyer, Fidel Castro, who would become the country's President. Starting in 1959, the new Revolutionary government enacted radical reforms that focused on nationalization and redistribution (8), revealing a "socialist humanist vision" at its core (12). The vision was shaped primarily by Martís writing during the independence movement of the 1860s, by Guevara's writing throughout the revolution itself, and by Fidel Castro's speeches. Eventually, this philosophical orientation led to a close economic relationship with the Soviet Union (16), which had disastrous effects when the Soviet Union fell in the 1990s, leading to the "Special Period in a Time of Peace" (Brenner et al. 2008, 1). Cuba's socialist orientation and the anti-imperialist position of the revolution also led to the U.S.'s many efforts over the years to overthrow or undermine the revolutionary government (18). The U.S. trade embargo, started in 1964 and meant to destabilize Castro's government (19), exacerbated the economic crash that led to the Special Period, and is still in effect today.

From the very beginning, education and health were the priorities of the new revolutionary government (Breidlid 2007, 621), and this has continued through the trials of the Special Period and the trade embargo. Even as the Cuban economy declined, the rights to education and health were upheld in a way that has arguably not been seen 
anywhere else, and the gains that have been achieved even by the standards of those who oppose the revolution are undeniably exceptional. However, even with the success of the Cuban system, development practitioners, policy makers, and theorists continue to marginalize the epistemic framework from which it stems and appear to discredit the approach's feasibility despite its evident success.

\subsection{The Epistemic Framework}

At the heart and foundation of the revolution is its philosophy. It was and is a revolution of ideas, led by those who felt a need for change not only in the material realities but also the mental realities of the Cuban people. That transformation of thought can be traced back to the late 1800 s, when the poet Martí was writing and fighting in the Cuban war for independence. The threads of Martís thinking were picked up in Guevara's writing during the Cuban revolution, and Guevara and Castro enacted it as an explicit strategy to transform Cuba. The epistemic framework of the Cuban revolution has been shaped by many theorists and ideas, not just Martí and Guevara, but it is these two intellectual giants that are most often mentioned as the keystones of Cuban revolutionary thought. As such, they will be my focus.

Martí's Our America provides a clear example of his philosophy and how it has been passed down to contemporary Cuba. He puts the fight for knowledge at the forefront of the Cuban struggle for independence, saying that "the trenches of ideas are worth more than trenches of stone" (Martí 2002, 288) and that "anyone who deliberately or unknowingly sets aside a part of the truth will ultimately fail because of the truth he is lacking" (291). This makes concerns about epistemic injustice particularly relevant to the Cuban epistemic framework, which has arisen from Martí's philosophy. As Susan 
Babbitt points out, "the independence movements of the nineteenth century and the Cuban revolution of the twentieth were about epistemic injustice, long before it was discussed in the U.S." (forthcoming, 266), because both Martí and Guevara were concerned with the domination of ideas as an aspect of the domination of people. Martí in particular saw liberation from the European liberal conceptions of the self as necessary, "not only for moral reasons but for the sake of human potential" (262).

Although knowledge was central for Martí, it was a different conception of knowledge than is usually used by the West. Martí's conception of knowledge is an embodied knowledge, resting on the idea that "the body thinks" (263). This demonstrates a causal, naturalistic conception of knowledge (268). He viewed all aspects of knowing as "radically contingent upon circumstances and conditions" of the knower (263), an insight that has only recently been echoed in Western philosophy in the work of some feminist standpoint theorists and evident in Code's ecological thinking. Babbitt connects this kind of embodied view with Karl Marx and his dialectic materialism, and she argues that for both Martí and Marx this view means that "we must change the world, even ourselves, to know real human needs. Whether our beliefs about such needs are true depends on how we act and for what purpose" (263). As a result, knowledge is a matter of contextual cause and effect. According to Babbitt, Marx's "naturalistic, realist view of knowing says knowing is dialectical" and this means "the world acts upon us and we receive back” (Babbitt 2017). Furthermore, Martí views human beings as part of and dependent on nature, which includes our fellow human beings (Babbitt forthcoming, 265). This view of human beings is reflected in Code's ecological thinking. 
Martí also believed that the European intellectualist view of knowledge was a barrier to education and to gaining knowledge (Babbitt in an email to Christine Koggel March 15, 2017), because epistemic freedom required "transforming- politically, socially and personally- conditions explaining it: capitalism and imperialism" (Babbitt forthcoming, 268). The European intellectualist tradition has been shaped by those capitalist and imperialist conditions. Martí viewed education as critical to the sort of transformation that would lead to developing better citizens (2002, xi). He included in the right to education both women (156) and "Indians" (160), going so far as to claim that "to be educated is the only way to be free" (Martí 1884) and extending that freedom to all people. To achieve the desired transformation, "education" also had to be culturally appropriate, tailored to the context is which it is occurring and to the people who are being taught (Martí 2002, 160, 291). He believed it had to be embodied in practice and embedded in context, a belief reflected in his statements that "to know is to solve" (290) and "to think is to serve" (296). The value of service to others, not for personal monetary gain but for the well-being of all human beings, is also clearly reflected in his writing (Babbitt forthcoming, 265). This view of education is reflected in the education system that was enacted by the revolutionary government and continues in modern day Cuba. Martí's thought was also very anti-imperialist (Martí 2002, 292). He called the encroaching influence of countries like the U.S. "a tiger on velvet paws". It is an insidious threat that has shaped how Cubans see themselves, often in unnoticed ways (Babbitt forthcoming, 263). This warning is reminiscent of Bhargava's argument about the colonization of epistemic frameworks, discussed in section 4.4. Martí saw ideas as one of the main battlegrounds of the revolutionary war for independence in the $1860 \mathrm{~s}$ 
(264), and it continues to be one of the main struggles of the current revolutionary government. Because of his focus on ideas, Martí viewed the fight for Cuban independence as being about the importance of using embodied and contextual knowledge to situate a history and a people in that place, something that is always lacking in imperialist control. He claimed that an emerging country "demands forms that are appropriate to it" (Martí 2002, 290) and that "to govern well, one must attend closely to the reality of the place that is governed" (290). As a result, "the battle is not between civilization and barbarity, but between false erudition and nature" (290). Similarly, the struggle is not primarily at the level of "developed" versus "developing" but between the imposition of dominant epistemic frameworks and the cultivation of indigenous, alternative ones. Developing alternative epistemic frameworks through an education based on dialectic materialism will not only be about the creation of ideas but will also be about loss (Babbitt 2017). This is because when something new is learned, it will almost always result in something that one believed or thought they knew being lost. Furthermore, if thinking is embodied, then acquiring new knowledge not only changes what one knows but also who one is, resulting in the loss of aspects of identity. Knowledge, for Martí, is about change. This also is reminiscent of Lugones' logic of curdling, showing how knowledge can be both gain and loss, to different degrees and in different ways, always changing.

Guevara's philosophy picks up on many of the aspects of Martí's. Like Martí, his writing is explicitly and emphatically anti-imperialist. Guevara's anti-imperialism, however, was more antagonist to U.S. imperialism specifically. This led in part to Cuba's identification with socialism, although Guevara often connected Cuba's struggle to the 
plights of other "developing" countries rather than with other communist countries in the Soviet bloc. The need for internationalism and "fraternal attitude" among the less powerful and economically wealthy countries in the world is emphasized throughout his work (Guevara 2002, 18-19), and he defines development as meaning an escape from imperialism and outside economic domination (18). Knowledge, in the same embodied sense as it is used by Martí and reflective of the influence of Marxist dialectical materialism, is also central to Guevara's philosophy. He builds on these ideas to create a new vision of what it means to be human, one that he explicitly connects to the Cuban revolution.

Guevara also argued that "to build communism it is necessary, simultaneous with the new material foundations, to build the new man and woman" (34). Education was a key part of that building process (35), because it was necessary to develop through conscious habit "a consciousness in which there is a new scale of values" that responds to moral incentives (35). The goal is to reshape human beings as moral beings with different values than the ones expected by the dominant world view of the West. The transformation would require not only formal education, but also self-education (35). With that new consciousness, work "becomes an expression of oneself" (38) and never a punishment (43), "artistic inquiry experience[s] a new impulse" (40), and the individual becomes more complete through responsibility and "inner wealth" (43). The "new man and woman" is achieved when "love of living humanity is transformed into actual deeds, into acts that serve as examples" (44). Guevara envisioned a revolutionary person, who understands that "the pride of serving our fellow man is much more important than a 
good income; that the people's gratitude is much more permanent, much more lasting than all the old one can accumulate" (Guevara 2003, 117).

In this vision of the Cuban revolution, the individual is still important (Guevara $2002,30)$. However, that individual is not an isolated, autonomous agent like the one assumed in liberal individualism. Individuals are but one aspect of the "multifaceted being" that Guevara refers to as "the mass" (31). He argues that within the revolution, the individual is not subordinated to the state in the kind of homogenizing way that opponents assumed (31). Instead, they take the initiatives of a leader like Fidel Castro, and "make it their own" (31). Guevara describes the effect as a "close dialectical unity between the individual and the mass, in which both are interrelated and, at the same time, in which the mass, as an aggregate of individuals, interacts with its leaders" (32). Lugones' logic of curdling, described in section 3.2, can help us to understand how this is the case. The individual can be both an individual and an element of the mass, without being subordinated to the whole. Western thinking, where the state and the individual are necessarily separate in order for the individual to be free, does not allow an understanding of what Guevara is describing as the experience of the revolution.

The health care system of Cuba can be traced to directly Guevara and his vision of the "new man and woman". Trained as a doctor (Guevara 2003, 112), he believed that there is a right to health care for all citizens, and that it was the doctor's obligation to put "the technical knowledge of his profession at the service of the revolution and of the people" (113). He also argued that "the life of a single human being is worth millions of times more than all the property of the richest man on earth" (117) and that the greatest treasure a doctor could have was "the people's gratitude" (117). This set the foundation 
for a health care system in which the moral incentives of providing care to other Cubans was seen as far more valuable than monetary compensation, and where medical professionals were expected to be prepared to use their skills wherever they were needed across the country or around the world. He viewed the revolution itself as "the greatest work of social medicine that has been done in Cuba" (114), and thought that the consciousness of the "new man and woman" would arise among the health care professionals (113). He argued that when poor, rural people who had not previously had access to education graduated as new doctors, the desire to be payed extra would not be the motivating force for going back into the countryside. Instead, they "would [request] the posts with the most responsibility and most work, in order to show that the years of study given to them were not in vain" (116-117). He also thought that to "get to the heart of the medical question", doctors needed to go to the places where people required treatment to get to know them, "find out what diseases they have, what their ailments are, what extreme poverty they have lived in over the years" (115).

This section has only covered the elements of the Cuban revolution's epistemic and philosophical background that are most relevant to the topics of health care, education, and internationalism, and which come from the two most prominent figures who contributed to shaping that background. As a result, a great deal more could be said about Cuba's epistemic framework than has been said here. However, from this brief discussion it should be clear how different the Cuban hermeneutical resources are from those coming from the dominant global institutions and countries in the West.

The Cuban vision is one of dialectic materialism, deeply embodied and embedded in relationships shaped by the particular context of Cuba and its colonial history. The 
vision also pushes for something new, a creative process of discovering what it truly means to be human and what it means to know. That process will involve some loss not only of belief but of self. It will require understanding that while the individual is important, it cannot be disconnected from the mass of its relationships. This epistemic framework clearly has many connections to the approach to epistemic injustice that I presented in Chapter 3, and is drastically different from the approaches described in Chapter 4. It also should be evident why this alternative framework for understanding human life itself is seen as threatening by the dominant global world view. Accepting its credibility would require questioning almost everything that the dominant epistemic framework claims, a loss that those in power are not willing to risk. However, when one examines how the Cuban epistemic framework has impacted the well-being of the Cuban people, it can be seen how the loss of knowledge caused by the marginalization of these alternative hermeneutical resources is detrimental to understandings of development and to the world more generally.

\subsection{The Cuban Education System}

The Cuban education system is one such area where the positive influence of the revolution's epistemic framework is evident. In 1953, before the revolution, around a quarter of the Cuban population was illiterate (Breidlid 2007, 620; De Quesada 2011, 138; Fitz 2016, 46). Even more concerning, however, was the fact that in rural areas the proportion was 41.7 percent. The poor and marginalized were being excluded from the education system because of high school fees and pervasive corruption in Batista's government (Breidlid 2007, 620). Moreover, structural inequalities and regional demographics meant that this exclusion was in large part racialized (Breidlid 2007, 628). 
The corruption and the inequality evident to marginalized groups in the education system was among the many causes of popular support for the revolution, and resolving these issues was "the number one priority of the Revolutionary government" (De Quesada 2011, 137). This involved not only reshaping the system, but reshaping the people who had been shaped by the inequalities and structural injustices the old system imposed.

The Cuban education system is quite obviously built on a foundation of Martí and Guevara's visions. After the success of the revolution, one of the first initiatives of the revolutionary government was the literacy campaign (López 2011, 58), based on an idea Martí first proposed in 1884 (Martí 2002, 357). In 1961, thousands of students "armed only with a lantern and a literacy booklet" (De Quesada 2011, 139) were sent out across the country, even to the most remote areas, and "in a single year, more than 707, 000 people were taught to read and write" (Fitz 2016, 46-47). This set the foundation for the reforms that followed, where the regime sought to "establish schools across the country and to train teachers to work in all corners of the Island, to fully transform and consolidate the Educational System in both its objectives and content and to diversify education and multiply the number of universities" (López 2011, 58). The goal was to fulfill the right of education for all Cubans regardless of gender, race, location or income. To further that goal, one of the revolutionary government's early initiatives was a Scholarship Plan to help with the moving costs of rural students who couldn't by themselves afford to travel from remote cities to attend university in Havana (De Quesada 2011, 137). At the same time, attempts were made to "create university campuses throughout the country" and to increase domestic university level training in fields, such as economics, that had previously required one to be educated in other 
countries (137-138). These early initiatives reflected the revolutions' commitment to a right to education. This education extends beyond formal, intellectualist education to a more embodied, moral education of the kind that Martí and Guevara envisioned, and is sensitive to the context, history, and conditions of the students.

Many changes have been made to the education system over the half century that the revolutionary government has been in power. However, some principles have remained the same, and were enshrined in the Constitution of the Republic of Cuba in 1976 (López 2011, 55). Education is free, from kindergarten all the way through to university (Breidlid 2007, 628). This is reflected in Article 9 of the constitution, which states that "the State, as the Power of the people, guarantees... that there will be no child lacking a school, food and clothing; that there will be no youth lacking the opportunity for study; that there will be no one lacking access to study, culture and sports..."' (The Constitution of the Republic of Cuba 1976, Article 9). Through this principle, the constitution guarantees education as a right for everyone and gives the government the responsibility to provide access to that education "without regard to age, gender, ethnic or religious differences" (López 2011, 57). In carrying out that guarantee, the Cuban government has focused on expanding education to previously marginalized groups. Although these inequalities have not been eliminated, Cuba has made great strides in mitigating the disadvantages caused by the institutional and structural inequalities stemming from the country's colonial history (Breidlid 2007, 628).

The education system also focuses on combining study with work (López 2011, 57; Breidlid 2007, 62-626), reflecting Martí's claim that “to think is to serve” $(2002,296)$ and funneling students towards careers that contribute to "the country's development 
needs within five years" (López 2011, 62). Although this focus on the needs of the country may limit the choices individual students have, as was evident to me from conversations I have had with Cuban students while visiting Havana, it has merits. These limits help to ensure that the need for professionals in areas such as teaching and health care are fulfilled. The fact that there may be Cubans who "see" it as a limitation of choices reflects both exposure to the values in liberal societies as well as a curdling in the perspectives of individual Cubans that is ongoing and changing. Overall, Cuba has created an extremely well organized system (Breidlid 2007, 626), focused on inclusion and equality of opportunity (618), with a population that seems to have internalized a view of education as liberating and revolutionary (626).

According to the philosophy of the revolution, education is "a task in which everyone participates in one way or another, and therefore should be the responsibility of all” (López 2011, 55). However, problems for the education system have arisen since the fall of the Soviet Union in the early 1990s (Breidlid 2007, 622). The Soviet Union had been Cuba's main trade partner and source of economic support, subsidizing oil prices (Brenner et al., 112) and providing over 60 percent of Cuba's trade from 1978 until the collapse of the Soviet bloc (113). With the fall of the communist regime, the economic situation in Cuba declined dramatically (Breidlid 2007, 622). Because of the trade embargo imposed on Cuba by the U.S. since February 1962 (Brenner et al., 18), which prevented most countries from trading with Cuba, the revolutionary government had to make a hard choice. Either capitulate to the U.S. demands to remove Fidel Castro, denationalize industry, and implement a multiparty system; or find an alternative source of revenue. To try to rescue the failing economy, the Cuban government eventually turned 
to the tourism industry to bring in foreign currency, and in the process opened a capitalist sector (Breidlid 2007, 622). Although it succeeded in boosting the economy, it had the side effect of resulting in many teachers leaving education for the higher salaries of the tourist industry (Breidlid 2007, 622; Kirk 2008, 300). It has also resulted in a reduction of the esteem given to teaching. Although many of the newest teachers still seem to be ideologically committed to the goals of the revolution, concerns about quality and resources in the education system have become pressing (Breidlid 2007, 632).

This crisis in education has led the Cuban government to enact changes to the system, first in 2001 (Breidlid 2007, 623) and then again in 2009-2010 "with the goal to continue improving the quality of education so that current and future generation are increasingly prepared to face and solve the problems generated by development, and to do so with strong patriotic and revolutionary convictions" (López 2011, 67). Anders Breidlid expresses concern over the very strong emphasis on conformity to what he calls the "ideology of the revolution" that seems to permeate the education system $(2007,618)$, leading to what he sees as the ostracization of non-conformist views (626). He argues that this seems to have increased with the perceived threat to revolutionary values when capitalism began to gain ground in Cuba through the tourism industry.

Evident in Breidlid's concerns is the influence of the language of the dominant hermeneutical resources and epistemic frameworks of the West. Although most of his discussion is sympathetic to Cuba, he still discusses education from a perspective and framework that is antagonist towards that which builds on the philosophies of Martí and Guevara. His discussion is imbedded in the assumptions of the dominant global institutions and their epistemic framework. As I mentioned in section 4.4., on Bhargava's 
view of the colonization of epistemic frameworks, resisting the domination of such frameworks is very difficult. In this case, hearing the alternative perspectives of the Cuban revolution may require quieting the voices that are embedded in the globally dominant world view. Furthermore, the vision of the revolution is one of the transformation of people, a process that requires a certain kind of education that would not have been seen as such by Westerners such as Breidlid. More will be said about the struggle to maintain the values and ideology of the revolution in the face of encroaching capitalism in section 5.5.

Despite these challenges, the success of the Cuban education system according the standards of the dominant institutions have surpassed all expectations and all other countries in Latin America and the Caribbean. In 1964, the country was declared a ‘territory free of illiteracy' by UNESCO (Breidlid 2007, 621). In 2015, Cuba was the only country from Latin America and the Caribbean that reached all the "Education for All (EFA) goals set in 2000" (UNESCO 2015a, 1), having reached those goals before they were even set (De Quesada 2011, 139). It achieved a ranking of 28th of the 113 countries counted on the EFA Development Index in 2012 with a 0.981, compared to the U.S.A. at 36th with 0.975 . The closest Latin American country to Cuba's ranking was Uruguay, ranked 40th with 0.969 (UNESCO 2015b, 231). Improving the lives of Cuban people who were most marginalized before the revolution has been the main goal of the revolutionary government's policies in education, and they have succeeded by the standards of the dominant global institutions (Breidlid 2007, 621). All of this has been accomplished with no help from the IMF or WB, while resisting "the globalized drive for privatization" (629), and has been 
maintained in the midst of the economic crisis caused by the fall of the Soviet Union and U.S. trade embargo (629). This success, in the face of great adversity and active attempts at obstruction as well as the difficulty in resisting the influence of globally dominant frameworks, "seems to undermine the conventional wisdom that only well functioning countries in the north can provide quality education for the majority of their pupils" (619). It has gone counter to the "global reform environment of privatization, downscaling of the state role, and cost recovery" (Gasperini 2008, 299), but not even the biggest critics of Cuba's political system can deny the success of its education policies, even by the standards of those critics (Breidlid 2007, 620).

However, the standards of success imposed by the dominant global institutions do not tell the full story of Cuba's revolution in education. The achievements of the Cuban education system cannot be measured solely in empirical terms or effective results (like Code's). A significant part of the success of the Cuban education system is that it reflects the vision that Martí and Guevara had of Cubans as a new kind of moral and political person. The benefits of the values of the revolution, which are taught alongside the skills by which the dominant global development institutions measure success, is clear even among those Cubans who do not agree with the philosophy and vision of the revolution. The revolution of ideas has made a mark.

\subsection{The Cuban Health Care System}

The revolution of ideas is also clear in the Cuban health care system. According to Fidel Castro, before the revolution, the health care problems in Cuba included "the absence of a national public health plan, semi-official and private services that were better than those provided by the government; an orientation toward curative medicine; abandonment of 
rural and some urban areas; individual medicine; mercantilism; competition between private services; administrative centralization with a public unaware of treatments that could benefit them" (Castro quoted in Fitz 2016, 45). It should be noted that these characteristics describe the approach to health described in the cases of Chagas disease and Tanzanian health care discussed in section 4.3. Don Fitz adds that during the Batista era there was "no reliable data on health indicators, an insufficient number of doctors and dentists being trained, and severe underfunding of what few research facilities existed" (Fitz 2016, 45). Furthermore, "the pharmacy industry was 70 percent foreign controlled, and created many products lacking treatment value. Only 10 percent of children were covered by specialized pediatric care. Vaccination programs were unavailable" (45). With this litany of impediments to effectively addressing the health of the Cuban people, it is no wonder that establishing quality health care was of great concern. What Fitz chooses to point out in listing these impediments is obviously influenced by the priorities and perspective of the U.S., however it still has some value in showing the importance of the gains of the revolution even from a Western perspective. The value that the Cuban revolution placed on these factors is evident from the attempts that have been made to address them, but that value is not only about effective results. It is also about rights and humanity.

Guevara's philosophy towards health care is clear in the revolutionary government's health care system, a system that is also enshrined in the 1976 constitution. The system follows the principles, based in Guevara, that "health care is a right, available to all equally and free of charge" and that providing it is "the responsibility of the state" (Keck and Reed 2012, 14). The system is highly structured and well organized, and 
focuses primarily on preventative rather than curative medicine (Campion and Morrissey 2013, 298). Doctors and nurses live in the communities they serve, are available at all hours (Suri 2016, 641), and do home visits at least once a year to approximately 100-200 families they are assigned (Suri 2016, 642; Campion and Morrissey 2013, 297; Loewenberg 2016, 327).

As a result of their residency in and personal relationship with the community, the medical team knows details about the lives of their patients which allow the implementation of "comprehensive prevention and healing through longitudinal relationships between physician and patient, woven into the fabric of the community" (Suri 2016, 642). This curative, community, and relational focus is what is missing in the approach to health discussed earlier in the cases of Bolivia and Tanzania, and it demonstrates an implementation of the kind of contextual and relationship-based analysis that I emphasize in Chapter 3. It recognizes the deep interconnection between the many aspects of peoples' lives that can impact their well-being, as well as the important role that relationships which reflect moral responsibilities to others play in addressing those many aspects of well-being. It also draws on the Marxist dialectic materialism that is central to the kind of socialism that has resulted from the philosophical foundations of the revolution. Using this dialectic materialism, Cuba has created a health system that is reminiscent of the kind of embodiment that is central to Code's ecological thinking, but it is at bottom their philosophical enactment of dialectic materialism. There is a heavy focus in this comprehensive approach on understanding "how social and economic factors influence the health of society" (641), further reflecting a more context based, embodied and material approach to medicine. 
This health care system exists not only within the capital of Havana, but across the country. Medical teams are sent to marginalized urban communities, rural areas, and the eastern part of the island in order to ensure that everyone has access to the services needed to fulfill their right to health care. In ensuring access even in remote areas, the health care system represents further steps on the part of the revolutionary government to fight structural racism, since many of those remote areas were predominantly black (Fitz 2016, 53). Guevara's image of the revolutionary doctor was reflected in the "eagerness to go to the countryside" (49) that became evident among many practicing physicians. New medical students also showed "the expectation that they would be trained not for personal gain, but according to the needs of society" (49).

As with the case of education, the fall of the Soviet Union had extremely detrimental effects on the resources available for the health care system, and has led to the epistemic framework on which that system is based being threatened by the global hermeneutical dominance of outside world views (specifically that of the U.S.). In addition to experiencing a brain drain similar to that in education as medical professionals sought more lucrative positions in the tourism sector, the U.S. economic embargo prevented access to essential medicines and basic equipment (Suri 2016, 641). In response, however, Cuban doctors have found extremely inventive solutions, particularly to outside eyes, using everyday items to replace the equipment they cannot access (642) and innovating new approaches (DeVos et al. 2008, 289). In addition, the country has developed its own pharmaceutical industry, with so much success that it not only manufactures its own pharmaceuticals but also exports them (Campion and Morrissey 2013, 299; De Quesada 2011, 138). 
Critics of Cuba's health care system argue that "the system is not designed for consumer choice or individual initiatives" and that there is no private health system as an alternative to the government run one (Campion and Morrissey 2013, 298), assuming that these things are necessary and always desirable. However, as Babbitt points out in her critique of outsider discussions of Cuba, although it is possible "that what matters most to the realization of human worth is choice" this belief is by no means certain (Babbitt 2006, 298). To assume that choice and, more specifically, a certain conception of choice is what is most important in human life is an injustice to alternative perspectives. The critics that condemn Cuba's health care system based on it lacking the full range of choice available to consumers are very obviously drawing on an epistemic framework based in the dominant hermeneutical resources of the U.S. and the West more generally.

Despite this critique, the lack of choice understood as opportunity to choose from a range of consumer goods seems to have minimal negative impact on the quality of health care provided in Cuba, which is "ranked near the top of those countries on course to meet the United Nation's Millennium Development Goals" (Keck and Reed 2012, 17). More specifically, in the 2010 "Millennium Development Goals Report Card: Measuring Progress Across Countries," Cuba is listed as one of the top 20 countries in terms of relative progress towards the Millennium Development Goals (MDG) (Millennium Development Goals Report Card 2010, 9). Compared to the U.S., the country achieves lower infant mortality, higher vaccination rates, and comparable life expectancy (Campion and Morrissey 2013, 298). In the same 2010 MDG report, Cuba's under five mortality rate was 7 per 1,000 live births (Millennium Development Goals Report Card 2010, 89), it had a 99 percent immunization record for measles (Millennium 
Development Goals Report Card 2010, 89), and it had a 95 percent access rate to HIV treatment (Millennium Development Goals Report Card 2010, 93). Although the 2010 MDG Report Card did not contain comparable data for the United States, this data is still significant evidence of Cuba's health care success.

This success, which is once again being measured by the standards of the dominant global institutions and does not necessarily consider everything that is valued within the Cuban epistemic framework, is even more noteworthy considering "the trends in many other countries towards implicit privatization through health sector reform since the early 1990s" (DeVos et al. 2008, 289) and toward corporate monopolies of the pharmaceutical industry in the rest of the world (De Quesada 2011, 138). Cuba's empirically measured success according to conventional views within dominant global institutions about what good health care is has been achieved with a system that runs counter to the conventional views about how good health care should be achieved. However, like in the case of Code's focus on epistemic effectiveness rather than epistemic justice in the case of Tanzania, this focus on globally dominant measures of effectiveness do not reveal the whole story.

\subsection{Cuban Internationalism in Education and Health}

Ricardo Alarcón De Quesada, former Cuban ambassador to the UN and president of Cuba's National Assembly, claims that internationalism is "the heart and substance of socialist ideal" (De Quesada 2011, 139). This is clear in Guevara's emphasis that "proletariat internationalism is a duty, but it is also a revolutionary necessity" (Guevara $2002,44)$. Guevara often wrote about it, and incorporated it into many of his speeches. He also lived it, having been born in Argentina rather than Cuba, and then lending his 
skills to revolutionary efforts in the Congo and then in Bolivia, where he died. His passion for creating an alliance among developing nations in what he called "the struggle against imperialism" (17) as well as "the struggle against backwardness and poverty" (18) is reflected in the extension of Cuba's health and education policies abroad.

"Yo Sí Puedo", a method of "building literacy around the needs and initiatives of communities themselves, working with people to read the word and the world" has been used to carry out literacy campaigns throughout Latin America using a strategy similar to the one that transformed Cuba (De Quesada 2011, 140). This internationalism is even more evident in connection to health care programs than it is in education. For many years, "tens of thousands of Cuban doctors have provided their services in many places in Latin America, the Caribbean, Africa and Asia (140). This includes disaster relief in Haiti and Pakistan (140) as well as being on the front lines of the Ebola outbreak in 2016 (Loewenberg 2016, 328). Willingness to take part in such international missions usually reflected the degree to which the Cubans involved were supportive of the changes being made by the revolutionary government (53). They had begun the transformation into the "new man and woman" that Guevara saw as a necessary part of the revolution, embracing a new way of understanding the world.

In 1998, the Escuela Latinoamericana de Medicina (ELAM) was founded to increase Cuba's contributions to internationalism in health care. The ELAM is a medical school that trains students from around the world "on the merit of their commitment to serve the vulnerable rather than gain personal fortune" (Huish 2009, 302). It is focused on training practitioners for poor, indigenous, and otherwise marginalized communities, providing "a first contact practitioner for every person" (Flegel 2009, 305), and has 
succeeded in training more than 26,000 students from more than 123 countries including the U.S. The students are given full scholarships for six years, as well as free room and board and a small stipend (Loewenberg 2016, 327).

Like the training of doctors for the health care system within Cuba, the ELAM curriculum focuses on prevention, community health promotion, and social and environmental factors. It also incorporates traditional and natural medicines "for therapeutic and preventative care, as long as the procedures can be tested and measured for harms and benefits" (Huish 2009, 302). The idea is to prepare the graduates for "the actual diseases they will encounter" and to train them "to identify, perhaps even to intervene in, the social, cultural and economic factors that create the disease incidence rate that they are encountering" (Flegel 2009, 305). Because the education is largely free, students are also able to study family medicine without the concern about it being low paying, a factor that usually dissuades students in many countries where they accumulate enormous debt during their studies (Loewenberg 2016, 328).

This applies the insights of ecological thinking to the international level, where the concern for context, embodiment, relationships, and the principles of dialectic materialism all come to light. Some of the insights and features include incorporating elements of the future doctor's local culture, wherever that local culture is, and ensuring that wherever they go to use their training they will be able to implement an approach that considers the social, political, economic, cultural, and natural environment they are in. The ELAM's approach also discourages the kind of categorization, labelling and ranking of medical specialization according to how well they pay that results in family or community medicine being disvalued. Instead, this view of health care considers family 
medicine as the most valuable area of health care because it is the foundation of and overlaps with all areas of health care. This also reflects the logic of curdling discussed in section 3.2.

Central to ELAM's approach is an emphasis on ethics that puts "community service above personal fortune" (Huish 2009, 301). This is demonstrated through the example of the instructors, many of whom are the living embodiment of Cuba's alternative epistemic framework and their commitment to internationalism through their own participation in the Cuban medical brigades providing care throughout Latin America and Africa (302). It is further engrained through prestige given to Cuban faculty teaching at ELAM, a prestige that is not demonstrated through monetary compensation (which is usually viewed as the measure of success in capitalist countries such as the U.S.) but through national peer recognition (302).

Although ELAM has received a great deal of praise, it has also faced criticism. The curriculum, with its focus on areas such as public health and family medicine that receive less attention in most medical schools, raises concerns for those who are used to using Western medicine as the standard that "other areas taught in regular medical schools are missed" (Flegel 2009, 306). However, those who raise such concerns often fail to recognize that the standard approach to Western medicine may not be the best one. They do not question whether the things that may be missing in Cuba are less important than the things that they include but other countries do not. The doubts about the curriculum, and the lack of recognition of the program from some countries, shows the dominant groups' unwillingness to consider the possibility that another approach to medical training and health care could achieve the same (or better) quality of 
improvement in well-being. There is an active effort to judge the Cuban system as less credible, a credibility deficit that is taken up by the countries that don't accept accreditation in the Cuban system. This results in the further marginalization of the Cuban approach from the resources available to the rest of the world, to the detriment of other countries still trying to find an effective approach to providing much needed health care to their populations.

Other concerns have been aired about whether the Cuban model, and therefore the practices being taught to students at ELAM, can be implemented in other countries. For example, a student from South Africa expressed worry that "implementing the prevention approach in her home country will be difficult, because the primary emphasis is on treating diseases as they occur" (Loewenburg 2016, 328). That treatment oriented approach is reflected in the cases of Chagas disease and in the Tanzanian health care initiative discussed earlier. Moving away from it would take the kind of sustained effort that was used to reshape the selves and values of the people in Cuba. That sort of reshaping is unlikely to happen anywhere else in the world in the current political climate. The same is true of other factors important to Cuban health care such as home visits, knowing the local community, and spending a long time with the patient (328).

Yet another factor that presents a barrier to implementing the Cuba health care model in other countries is that many of the lower and middle income countries, those usually labelled underdeveloped, "have lagging health care systems that are too underfunded to employ physicians on an long-term basis" (Huish 2009, 303), and may lack the infrastructure and consistency that comes with the one-party rule present in revolutionary Cuba. Some countries are interested in learning from Cuba's model (DeVos 
et al, 288), but questions remain about whether its implementation would work in places that did not have a socialist political and economic system. Often, these other countries have health care policies that have been shaped through the restrictions imposed by the structural adjustment programs of the IMF and WB, which reflect a neoliberal approach that cuts spending and reduces government oversight. This creates the opposite of the centralized organization that may be a key part of Cuba's success. It is also an example of the imposition of dominant, and colonizing, frameworks which has led to negative development outcomes. Part of Cuba's unique approach has been to resist outside forces such as the IMF and WB, but without that resistance it may be impossible to achieve gains such as those that Cuba has seen.

This is a reasonable concern, since the Marxist dialectic materialism that shaped the thinking of Martí and Guevara is foundational to the Cuban approach and how it has shaped Cuba. Without something similar to the material reshaping of Cubans and their thinking that has occurred through education, health care, and revolution more generally over the last fifty years, it may be impossible to access the same gains. This makes paying attention to the epistemic frameworks, hermeneutical resources, and philosophies that have shaped the Cuban system even more important. The epistemic injustice against the Cuban revolution's world view has prevented other countries from accessing their alternative approach, through methods that very much reflect what Bhargava identifies as the colonization of epistemic frameworks. Cuba's resistance to this colonization in a world increasingly influenced by the dominant framework has, at least in part, caused the U.S.'s antagonism towards the country. 
To this point we have discussed the ways in which Cuba's history of colonization by the Spanish, followed by imperialistic overseeing by the U.S., led to a revolution that was based on the desire to reshape what it meant to be Cuban, shaping a "new man and woman" based on the vision and philosophy of Martí and Guevara. This new consciousness at the center of the revolution employs different system of values, which emphasize moral incentives over material incentives and work as essential for forming the self. From the philosophy that this new consciousness employs, Cuba has built systems of education and health that defy the expectations of outsiders who are drawing on conventional views based in a dominant hermeneutical resource shaped by the influence of the U.S. and neoliberal policies throughout the developing world. The successes of the Cuba system are based on paying attention to relationships and contexts, looking at the subject's embodiment in their environment and using that situatedness to determine how best to improve each person's well-being while also ensuring the wellbeing and continuation of the revolution as an alternative system. However, there is a great deal at stake in maintaining that alternative system and the alternative epistemic framework on which it is built. Prejudice against that alternative, because of its roots in communism but also because of the threat it poses to the current global structures of power and privilege, leads to the marginalization of the Cuba perspective. As a result, the important contribution that Cuba's effective approach to health and education could make to the development of other underdeveloped countries is buried, dismissed, and ignored. The last section of this thesis will describe the ways in which this has happened, and the role epistemic injustice plays in Cuba. 


\subsection{Concluding Remarks: A New Approach to the Global Role of Epistemic}

\section{Injustice}

There are some people, primarily critics but also those sympathetic to Cuba, who point out the instances of epistemic injustice that arise within the Cuban system. For instance, Breidlid argues that the authoritarian political system of Fidel Castro's revolutionary government does not allow much room for alternative epistemic frameworks within Cuba $(2007,627)$. Of course, he also recognizes that this happens even in "so called democratic countries" who also enforce a "relationship between the educational discourse and the hegemonic political discourse of the country" (Breidlid 2007, 627). This can be seen in the pro-capitalist and anti-communist discourses that still exist in the Canadian and American educational systems. In Cuba, the reinforcement of hegemonic views is just a bit more forceful. Although different opinions and views are accepted in classroom discussions, they need to be very well argued, and this allows teachers to pressure a classroom consensus agreeing with the official hegemonic discourse (626).

It does appear that this leads to epistemically unjust marginalization of views that are alternative to the dominant epistemic framework of within the Cuban system, and active attempts to make them appear less credible. However, an "alternative discourse" within revolutionary Cuba will more than likely reflect the dominant global discourse, at least on a philosophical level. Therefore, this apparent repression of a minority view within the alternative epistemic framework that is Cuban discourse could, in some situations, be a case of ensuring the survival of an alternative to the dominant global epistemic framework. In her discussion of the justification for one party rule in Cuba, Babbitt discusses Vladimir Lenin's sentiment that “the biggest mistake that could be 
made by the Communist Part in the Soviet Union would be to forget that the past still had to be overcome, to think that knowledge was secure, and that there wasn't still a constant battle to be fought against the heritage of past traditions" (Babbitt 2006, 299). The same sentiment can be applied to Cuba, where 50 years after the revolution, it is still trying to overcome the heritage of U.S. imperialism and domination.

This is why Cuba is an important case study to consider when discussing epistemic injustice in global development contexts. Cuba is a striking, ongoing example of how a colonized nation may need to react to the possibility that the dominant global epistemic framework is trying to colonize their epistemic landscape. Cuba's experience with the U.S. reflects Bhargava's account of the colonization of India. The imminence of the colonization of Cuba's epistemic framework is clearly evident in how dominant frameworks have encroached on policies that have been put in place, especially in the tourism industry, since the Special Period. The values of capitalism and of liberalism have infiltrated Cuba, and have already disrupted the gains of the Cuban system as doctors and teachers leave their jobs and opt instead for the monetary gains of the tourism industry. This is not to justify the philosophical repression and violations of freedom of speech that have occurred in Cuba. However, it is important to note that when theorizing about epistemic injustice on a global scale, the wrong and harm need to be examined in relation to a great number of factors and at all levels of relationships of power in a global context. The relationships among the factors being considered can greatly change how a situation should be analyzed.

For alternative hermeneutical resources to flourish, and for resistance to imperialist domination to be possible, it may sometimes be necessary to continue 
working at trying to push out the dominant way of looking at the world. This is why Cuba is an important case to look at in the context of global development, as well as in the context of epistemic injustice. It provides one real alternative to the hegemonic global approach to "development" that is used by global development institutions like the IMF and WB. In looking at the successes of Cuba's alternative approach, in the terms used by those dominant institutions, there are lessons that can be learned about the flaws in the dominant approach that cannot be seen through the application of the dominant epistemic framework alone. The epistemic injustices that are committed against Cuba and Cubans harm not only them, but also harm the rest of the world which could benefit from their insights.

There is a great deal of evidence of epistemic injustice imposed on Cuba and Cubans by the rest of the world. The attitudes of people from other countries towards many of the international efforts of Cuban trained doctors and teachers clearly reflect intentional credibility deficits and motivated marginalization imposed by those abroad on those who are trained in Cuba. When Cuba offered to send a medical brigade to Louisiana in the aftermath of Hurricane Katrina, President George W. Bush refused the help (De Quesada 2011, 140). As mentioned earlier, several countries including Argentina, Peru, and Antigua do not accept ELAM accreditation (Huish 2009, 303). In both of these cases, it appears that the skills and knowledge of Cuban trained doctors are given less credit based solely on where they were trained and not on their actual skills. There has also been little global media coverage of the efforts both in health and in literacy being done by Cubans around the world, including in "developed" countries such 
as Canada and New Zealand (De Quesada 2011, 141), which further marginalizes Cuba's alternative epistemic framework.

In each of these instances, there seems to be active resistance against acknowledging the value of Cuban contributions in areas of global development such as health and education. This further entrenches their marginalization on the global stage and in the production of knowledge in these areas, while also discrediting or silencing what they have achieved. There is an intentional epistemic injustice happening here, a motivated discrediting of an entire way of looking at the world in an attempt to erase it, even if it means losing the resources Cuba can provide to improving human well-being. In part, this prejudice reflects the history of U.S. and Cuban relations, and how it has influenced the world's view of the country. However, it goes beyond that. There also seems to be at play something like white ignorance. As discussed in section 3.1, white ignorance is an epistemology of ignorance where "a particular pattern of localized and global cognitive dysfunctions...produc[e] the ironic outcome that whites will in general be unable to understand the world they themselves have made" (Mills 1997, 18). Something similar is happening with Cuba. In Cuba's case, it is a pattern of cognitive dysfunctions that have the ironic outcome that the West is unable to understand the world it has made. It is an epistemology of ignorance that entrenches the supremacy of a part of the world, rather than a race. What Cuba faces is an epistemic injustice that includes all Fricker's categories (testimonial injustice, hermeneutical injustice, motivated ignorance, etc.) but which goes well beyond these as well.

There are some exceptions to this marginalization and discrediting, such as the New York Times praising Cuba's role in the Ebola crisis (New York Times Editorial 
Board, October 19, 2014), Pakistan and Haiti honoring Cuban doctors (De Quesada 2011, 14), and even the former president of the World Bank (James Wolfensohn) recognizing the achievements of the Cuban health and education systems (Lobe 2001). However, even with this verbal recognition, the IMF and WB have isolated Cuba "from the international arena of educational research" (Breidlid 2007, 618), not only depriving it of recognition and marginalizing it from the institutions that produce the knowledge incorporated into the dominant hermeneutical resources, but also depriving other countries who could gain a great deal from their insights and experiences. Although some recognition is better than none, it is inadequate to mitigate the wrongs of the epistemic injustice against Cuba and the harms it has caused.

The successes of the Cuban system, in health and education both at home and abroad, cannot be separated from each other or from the framework and philosophy from which they have emerged. Improved education results in improved health, which in turn increases the ability of individuals to gain an education. The improvements in both come from a different way of looking at the world and at human beings and this different way emerged directly and intentionally from the philosophies of Martí and Guevara. Paying attention to the relationships between health, education, and the philosophical context that makes the recognition and emphasis of this relationship possible is critical to understanding where Cuba is today. Those relationships stem from the particular history of the island, situated so close to the U.S. and shaped by a history of colonialism, imperialism, and dependency that never really went away even when it was "officially" independent. This history, dominated by attempts to pull free of the U.S. influence, is what led to the creation of a Cuban epistemic framework that tried to define itself 
independently of both the U.S. and the Soviet Union, and in opposition to imperialism around the globe. The Latin American philosophers who took the opportunity to enact their vision of a new man and woman can be said to have achieved a truly alternative epistemic framework, bringing with it alternative hermeneutical resources. However, it has also resulted in the continued marginalization of that epistemic framework on the global stage, and a credibility deficit when its achievements and even its vision are judged by the rest of the world.

From the point of view of a theorist trying to prevent (or at least mitigate) epistemic injustice, the Cuban epistemic framework and the political system that it spawned has many merits and many flaws. Among the many merits of the socialist humanism that is the philosophical backbone of the reforms made to improve the lives of all Cubans is its foundation in an egalitarian premise that tries to eliminate structural inequalities. In doing so, it should also eliminate many of the causes of systematic epistemic injustices, which stem from the marginalization of some groups from the institutions of knowledge production. Ensuring that all Cubans, no matter their race, gender, class or other social identities, are healthy and literate makes it far harder for anyone to be marginalized purely based on their social group. Equalizing the playing field in this way also means that prejudices that lead to unfair credibility deficits are less likely to arise in the Cuban system, because of the level of interaction between different social groups that should in theory and practice be occurring in the national health and education institutions.

The Cuban approach also focuses on incorporating an understanding of history and culture into both education and health, making it extremely contextual and 
demonstrating an understanding of how history, culture, economics, and politics relate to and influence each other. In education, "the indigenization of the discourse" ensures that students are learning about their own history, their own culture, and not according to "the modernist educational discourses in most Sub-Saharan African countries, often completely devoid of any link to the culture and context of pupils in schools" (Breidlid, 628). That same modernist educational discourse has been used in Canada with regards to our indigenous peoples, and the results have been extremely harmful not only to members of indigenous communities but also to their relationships with other social groups within Canada who understand nothing of their shared history of structural inequality and oppression.

This contextual and relationship focused approach is what is missing in the cases of Bolivia and Tanzania, discussed in section 4.3, and in most approaches to global development. The Cuban approach to education and health care takes on the sort of embedded and embodied approach that Code talks about as ecological thinking, where patients are understood in their contexts and in their relationships. To avoid epistemic injustice, this should include not just the physical relationships but also the relationships that people have to their history, their culture, and their epistemic frameworks and hermeneutical resources. In Cuba, attention is being paid to all of these relationships, and such attention needs to be taken on by global development in the rest of the world. The logic of curdling may help to allow the rest of the world to take on some of the lessons that can be learned from Cuba. The logic of curdling sees "the social world as complex and heterogeneous and each person as multiple, nonfragmented, embodied" (127). The dominant global development institutions need to stop seeing Cuba through the logic of 
purity, which reduces the country to a wholly dictatorial, anti-democratic government with an ineffective economic system. Instead, they need to see it as a complex nation with its own understanding of democracy and of human rights. Though these understandings are very different to the dominant view, they are still deserving of consideration and recognition. Insights from a curdled approach can help Cuba to be perceived not just as the enemy but as a place holding seemingly different values than the dominant ones that has something valuable to contribute. With such an approach, there can be room for epistemic justice and improved relations.

I have taken a new approach to epistemic injustice, diverging from Miranda Fricker's analysis in many ways. However, I think at the core we are both concerned with the same thing. When someone is wronged in their capacities as a knower, something ethically and epistemologically detrimental has happened that can result in many harms. When we look at those harms on a global scale, specifically through the lens of global development, what rises to the fore is the importance of relationships. Attention to relationships is important to understanding epistemic injustice, both its causes and its remedies. An approach is needed that pays attention to those relationships by employing ecological thinking and the logic of curdling to recognize the multiplicity, embodiment and interconnectedness of selves in a global context. Through this approach, it is possible to identify the importance of the relationships between people and the epistemic frameworks that they employ in navigating the world, and the grave harms that occur when frameworks that do not fit the dominant world view are treated in epistemically unjust ways. Too often, alternative ways of looking at the world, of understanding 
experiences, and of trying to find ways of improving well-being for everyone are discredited, marginalized, and ignored. When this happens, we all lose. 


\section{Bibliography}

Alcoff, Linda Martín. 2007. "Epistemologies of Ignorance: Three Types" in Race and Epistemologies of Ignorance, edited by Sharon Sullivan and Nancy Tuana, 39-58. State University of New York Press, Albany: NY.

—. 2010. "Epistemic Identities". Episteme, 7(2): 128-137.

Anderson, Elizabeth. 2012. "Epistemic Justice as a Virtue of Social Institutions". Social Epistemology 26(2): 163-173.

Babbitt, Susan. 2006. "Freedom and Democracy in Cuba: A Problem of Understanding", Moral Issues in Global Perspectives I: Moral and Political Theory, second edition, ed. Christine M. Koggel. Broadview Press Ltd.

_ 2017. “America's War, Failure of Democracy: What Happened to 'There are No Innocents'?". Global Research (http://www.globalresearch.ca/americas-warfailure-of-democracy-what-happened-to-there-are-no-innocents/5572381) (accessed March 18, 2017)

—. forthcoming. "Epistemic and Political Freedom". The Routledge Handbook of Epistemic Injustice, ed. Ian James Kidd, José Medina, and Gaile Pohlhaus Jr. Routledge University Press: 261-269.

Bailey, Alison. 2007. "Chapter 4: Strategic Ignorance" in Race and Epistemologies of Ignorance, edited by Sharon Sullivan and Nancy Tuana, 77-94. State University of New York Press, Albany: NY.

Beeby, Laura. 2011. "A Critique of Hermeneutical Injustice." Proceeding of the Aristotelian Society 111.3: 479-486. 
_. 2012a. "Reply to Jose Medina". The Social Epistemology Review and Reply Collective (social-epistemology.com): 1-4.

_ 2012b. "Collective Resources and Collectivity: A Reply to Jose Medina". Social Epistemology Review and Reply Collective 1 (11): 12-15. (http://wp.me/p1Bfg0ty)

Bhargava, Rajeev. 2013. "Overcoming the Epistemic Injustice of Colonialism”. Global Policy 4(4): 413-417.

Bohman, James. 2012. "Domination, Epistemic Injustice and Republican Epistemology". Social Epistemology 26 (2): 175-187.

Bondy, Patrick. 2010. “Argumentative Injustice”. Informal Logic 30 (3): 263-278.

Breidlid, Anders. 2007. "Education in Cuba—an alternative educational discourse: lessons to be learned?" Compare 37 (5): 617-634.

Brenner, Philip, Marguerite Rose Jimenez, John M. Kirk, William M. LeoGrande, eds. 2008. "Introduction: History as Prologue, Cuba Before the Special Period" in Reinventing the Revolution: A Contemporary Cuba Reader. Rowman \& Littlefield Publishers Inc., Plymouth: UK.

_.2008. "Economics" in Reinventing the Revolution: A Contemporary Cuba Reader. Rowman \& Littlefield Publishers Inc., Plymouth: UK.

Briggs, Charles L. 2011. “'All Cubans are Doctors!' News Coverage of Health and Bioexceptionalism in Cuba". Social Science and Medicine 73 (7): 1037-1044.

Campion, Edward W. and Stephen Morrissey. 2013. "A Different Model- Medical Care in Cuba". New England Journal of Medicine 368 (4): 297-299.

Coady, David. 2010. “Two Concepts of Epistemic Injustice”. Episteme 7 (2): 101-113. 
Code, Lorraine. 2006. Ecological Thinking: The Politics of Epistemic Location. Oxford University Press, New York: NY.

_ 2007. "The Power of Ignorance" in Race and Epistemologies of Ignorance, edited by Sharon Sullivan and Nancy Tuana, 213-230. State University of New York Press, Albany: NY.

. 2008. "Advocacy, Negotiation and the Politics of Unknowing". Southern Journal of Philosophy 46: 32-51.

Crenshaw, Kimberle. 1989. "Demarginalizing the Intersection of Race and Sex: A Black Feminist Critique of Antidiscrimination Doctrine, Feminist Theory and Antiracist Politics". University of Chicago Legal Forum 1989 (1): 139-167.

Dieleman, Susan. 2015. "Epistemic Justice and Democratic Legitimacy". Hypatia 30(4): 794-810.

Dotson, Kristie. 2008. "In Search of Tanzania: Are Effective Epistemic Practices Sufficient for Just Epistemic Practices?” The Southern Journal of Philosophy 46: $52-64$.

— 2012. "A Cautionary Tale: On Limiting Epistemic Oppression”. Frontiers: A Journal of Women Studies 33 (1): 24-47.

De Quesada, Ricardo Alarcon. 2011. “Cuba: Education and Revolution” Monthly Review 63 (3): 136-142

DeVos, Pol, Wim De Ceukelaire, Mariano Bonet, and Patrick Van der Stuyft. 2008. "Cuba's Health System: Challenges Ahead" Health Policy and Planning 23 (4): 288-290. 
Escobar, Arturo. 2007. "Actors, Networks, and New Knowledge Producers" in Cognitive Justice in a Global World: Prudent Knowledge for a Decent Life. Ed. Boaventura de Sousa Santos, New York: Lexington Books: 273-294.

Fitz, Don. 2016. “The 3000 Who Stayed.” Monthly Review 68 (1): 43-56

Flegel, Ken. 2009. "A Cuban Revolution in Medical Education: A Commentary on Huish 2009,”. Social Science \& Medicine 69: 305-306.

Fricker, Miranda. 2007. Epistemic Injustice: Ethics and the Power of Knowing. Oxford University Press, New York: NY.

— 2010. "Replies to Alcoff, Goldberg and Hookway on Epistemic Injustice". Episteme 7 (2): 164-178. Pdf.

—. 2013. "How is hermeneutical injustice related to 'white ignorance'? Reply to José Medina’s ‘Hermeneutical Injustice and Polyphonic Contextualism: Social Silences and Shared Hermeneutical Responsibilities'." Social Epistemology Review and Reply Collective 2 (8): 49-53. (http://wp.me/p1Bfg0-SC)

—. Forthcoming. "Epistemic Injustice and the Preservation of Ignorance" in The Epistemic Dimensions of Ignorance, eds. Rik Peels and Martijn Blaauw. Cambridge University Press.

Fuente, Alejandro de la. 2008. "Recreating Racism: Race and Discrimination in Cuba's Special Period" in Reinventing the Revolution: A Contemporary Cuba Reader, edited by Philip Brenner, Marguerite Rose Jimenez, John M. Kirk, and William M. LeoGrande. Rowman \& Littlefield Publishers Inc., Plymouth: UK.

Gasperini, Lavinia. 2008. "The Cuban Education System" in Reinventing the Revolution: A Contemporary Cuba Reader, edited by Philip Brenner, Marguerite Rose 
Jimenez, John M. Kirk, and William M. LeoGrande. Rowman \& Littlefield Publishers Inc., Plymouth: UK.

Goulet, Denis. 1973. The Cruel Choice. New York: Atheneum.

- 2006. Development Ethics at Work: Explorations- 1960-2002. Routledge, New York: NY.

Guevara, Che. 2002. Global Justice: Liberation and Socialism. ed. María del Carmen Ariet García. Ocean Press, Melbourne: Victoria, Australia.

- 2003. Che Guevara Reader: Writings in Politics and Revolution. ed. David Deutschmann. Ocean Press, New York: NY.

Harding, Sandra. 2006. “Two Influential Theories of Ignorance and Philosophy’s Interest in Ignoring Them”. Hypatia 21(3): 20-36.

Hoagland, Sarah Lucia. 2007. "Denying Relationality: Epistemology and Ethics and Ignorance" in Race and Epistemologies of Ignorance, edited by Sharon Sullivan and Nancy Tuana. State University of New York Press, Albany, NY: 95-118.

Hookway, Christopher. 2010. "Some Varieties of Epistemic Injustice: Reflections on Fricker". Episteme 7 (2): 151-163.

Huish, Robert. 2009. "How Cuba's Latin American School of Medicine Challenges the Ethics of Physician Migration”. Social Science \& Medicine 69: 301-304.

Keck, C. William and Gail A. Reed. 2012. "The Curious Case of Cuba." American Journal of Public Health 102 (8): 13-22.

Keet, André. 2014. "Epistemic 'Othering' and the Decolonisation of Knowledge". Africa Insight 44 (1): 23-37. 
Khader, Serene. 2011. Adaptive Preferences and Women's Empowerment. New York: Oxford University Press.

—. 2013. "Identifying Adaptive Preferences in Practice: Lessons from Postcolonial Feminisms". Journal of Global Ethics 9(3): 311-327.

Kirk, Margo. 2008. "Early Childhood Education in Revolutionary Cuba during the Special Period" in Reinventing the Revolution: A Contemporary Cuba Reader, edited by Philip Brenner, Marguerite Rose Jimenez, John M. Kirk, and William M. LeoGrande. Rowman \& Littlefield Publishers Inc., Plymouth: UK.

Kwong, Jack M. C. 2015. "Epistemic Injustice and Open-Mindedness". Hypatia 30 (2): 337-351.

Lobe, Jim. May 1, 2001. "Finance: Learn from Cuba, Says World Bank”. Website of the Inter Press Service News Agency. Available online at: http://www.ipsnews.net /2001/05/finance-learn-from-cuba-says-world-bank/ (accessed March 13, 2017)

Loewenberg, Sam. 2016. “Cuba's Focus on Preventative Medicine Pays Off” The Lancet 387: $327-329$.

López, Margarita Quintero. 2011. "Education in Cuba: Foundations and Challenges" Estudos Avancados 25 (72): 55-71.

Lugones, María. 2003. Pilgrimages/Peregrinajes: Theorizing Coalition Against Multiple Oppressions. Rowman and Littlefield Publishers: Lanham, Maryland.

_ 1987. "Playfulness, 'World'-Travelling and Loving Perception". Hypatia 2 (2): 3-19.

Maitra, Ishani. 2010. "The Nature of Epistemic Injustice". Philosophical Books 51 (4): 195-211. 
Malavisi, Anna. 2010. "A Critical Analysis of the Relationship Between Southern NonGovernment Organizations and Northern Non-Government Organizations in Bolivia". Journal of Global Ethics 6 (1): 45-56.

_. 2015. "Global Development and its Discontents: Rethinking the Theory and Practice”. PhD diss., Michigan State University.

Marsh, Gerald. 2011. "Trust, Testimony, and Prejudice in the Credibility Economy". Hypatia 26 (2): 280-293.

Marshall, S. E. 2003. “Epistemic Injustice the Third Way?”. Metaphilosophy 34 (1-2): $174-177$.

Martí, José. 2002. Selected Writings. Translated by Esther Allen. Penguin Books, New York: NY.

—. 1884. "Maestros Ambulantes". La América, New York.

Marx, Karl and Fredriech Engels. 1978. "Letters on Historical Materialism", in The Marx-Engels Reader, edited by R. Tucker. W.W. Norton: New York, NY: 760768.

Mason, Rebecca. 2011. “Two Kinds of Unknowing”. Hypatia 26 (2): 294-307.

McCollum, James. 2012. "Hermeneutical Injustice and the Social Sciences: Development Policy and Positional Objectivity”. Social Epistemology 26 (2): 189-200.

McKinnon, Rachel. 2016. “Epistemic Injustice”. Philosophy Compass 11 (8): 437-446. Medina, José. 2011. "The Relevance of Credibility Excess in a Proportional View of Epistemic Injustice: Differential Epistemic Authority and the Social Imaginary." Social Epistemology 25 (1): 15-35. 
_ 2012a. "Hermeneutical Injustice and Polyphonic Contextualism: Social Silences and Shared Hermeneutical Responsibilities" Social Epistemology 26 (2): 201-220.

_ 2012b. "Reply to Laura Beeby". The Social Epistemology Review and Reply Collective (social-epistemology.com): 1-3.

—. 2013a. The Epistemology of Resistance: Gender and Racial Oppression, Epistemic Injustice, and Resistant Imaginations. Oxford University Press, New York: NY.

_.2013b. "Color Blindness, Meta-Ignorance, and the Racial Imagination." Critical Philosophy of Race 1 (1): 38-67.

Mills, Charles. 1997. The Racial Contract. Cornell University Press, Ithaca: NY.

—. 2013. "White Ignorance and Hermeneutical Injustice: A Comment on Medina and Fricker". Social Epistemology Review and Reply Collective 3 (1): 38-43.

Origgi, Gloria. 2012. "Epistemic Injustice and Epistemic Trust”. Social Epistemology 26 (2): 221-235.

Ortega, Mariana. 2006. “Being Lovingly, Knowingly Ignorant: White Feminism and Women of Color". Hypatia 21 (3): 56-74.

Overseas Development Institute. 2010. Millennium Development Goals Report Card: Measuring Progress Across Countries. ODI Publications, London: UK. Pohlhaus Jr., Gaile. 2012. "Relational Knowing and Epistemic Injustice: Toward a Theory of Willful Hermeneutical Ignorance”. Hypatia 27 (4): 715-735.

—. 2014. "Discerning the Primary Epistemic Harm in Cases of Testimonial Injustice". Social Epistemology 28 (2): 99-114. 
Sherman, Benjamin R. 2016. “There's No (Testimonial) Justice: Why Pursuit of Virtue is Not the Solution to Epistemic Injustice”. Social Epistemology 30 (3): 229-250.

Singh, Aakash. 2013. "Deparochializing the Global Justice Debate, Starting with Indian Political Theory". Global Policy 4 (4): 418-419.

Stauffer, Jill. 2015. Ethical Loneliness: The Injustice of Note Being Heard. Columbia University Press, New York.

Sullivan, Sharon and Nancy Tuana. 2007. "Introduction" in Race and Epistemologies of Ignorance, edited by Sharon Sullivan and Nancy Tuana. State University of New York Press, Albany, NY: 1-10.

Sullivan, Shannon. 2014. "The Hearts and Guts of White People”. Journal of Religious Ethics 42 (4): 591-611.

Suri, Arjun. 2016. "The Art of Medicine. Social Medicine: Lessons from Cuba" The Lancet 387: 641-642.

The New York Times Editorial Board. 2014. "Cuba's Impressive Role on Ebola". The New York Times. Available online at: https://www.nytimes.com/2014 /10/20/opinion/cubas-impressive-role-on-ebola.html (accessed March 13, 2017).

Truth and Reconciliation Commission of Canada. 2015. Honouring the Truth, Reconciling for the Future: Summary of the Final Report of the Truth and Reconciliation Commission of Canada. Winnipeg: Truth and Reconciliation Commission of Canada.

Tuana, Nancy. 2004. "Coming to Understand: Orgasm and the Epistemology of Ignorance". Hypatia 19 (1): 194-232.

UNESCO. 2004. The EFA global monitoring report 2005. Paris: UNESCO 
—. 2015a. "Education for All 2000-2015: Only Cuba Reached Global Education Goals in Latin America and the Caribbean”. UNESCO, Paris/New Delhi. (en.unesco.org/gem-report/sites/gem-report/files/Latin_AmericaPR_en.pdf)

_.2015b. Education for All 2000-2015: Achievements and Challenges. UNESCO, Paris.

World Health Organization. 2017. (http://www.who.int/mediacentre/factsheets/ fs340/en/). Updated March 2017, accessed March 22, 2017, 7:00pm.

Yancy, George, ed. 2010. The Center Must Not Hold: White Women Philosophers on the Whiteness of Philosophy. Lexington Books, Plymouth: UK.

Young, Iris Marion. 1990. Justice and the Politics of Difference. Princeton, N.J.:

Princeton University Press.

1976. The Constitution of the Republic of Cuba 1976 (Amended 2002). (http://www.constitutionnet.org/files/Cuba\%20Constitution.pdf). Accessed March 2017. 